\title{
Paralelização de um modelo global de previsão do tempo em malhas localmente refinadas
}

Nelson Leonardo Vidaurre Navarrete

TESE APRESENTADA

$\mathrm{AO}$

Instituto De Matemática e Estatística

DA

Universidade DE SÃo PAUlo

PARA

OBTENÇÃO DO TÍTULO

$\mathrm{DE}$

Doutor EM CIÊNCIAS

\author{
Programa: Matemática Aplicada \\ Orientador: Prof. Dr. Saulo Rabello Maciel de Barros
}

Durante o desenvolvimento deste trabalho o autor recebeu auxílio financeiro da CAPES/CNPq/FUSP

São Paulo, dezembro de 2014 



\section{Paralelização de um modelo global de previsão do tempo em malhas localmente refinadas}

Esta versão da tese contém as correções e alterações sugeridas pela Comissão Julgadora durante a defesa da versão original do trabalho, realizada em 31/10/2014. Uma cópia da versão original está disponível no Instituto de Matemática e Estatística da Universidade de São Paulo.

Comissão Julgadora:

- Prof. Dr. Saulo Rabello Maciel de Barros (orientador)

IME-USP

- Prof. Dr. Alexandre Megiorin Roma

IME-USP

- Prof. Dr. Jairo Panetta ITA

- Profa. Dra. Claudia Inés Garcia

EACH-USP

- Prof. Dr. Pedro Leite da Silva Dias

LNCC-RJ 

"Un hombre puede ser destruido pero no derrotado" (Ernest Hemingway em "El viejo y el mar") 

Aos meus pais Sonia e Nelson, e às minhas irmãs Betsabé e Sonia, com todo o meu amor. 



\section{Agradecimentos}

Agradeço a Deus, que me ama, guia, apoia, ajuda e consola, muitas vezes sem o meu merecimento.

Ao meu orientador, Prof. Saulo, pela sua capacidade, competência, pela infinita paciência comigo, e principalmente por estar presente nos momentos mais críticos.

Aos meus pais, Sonia e Nelson, e às minhas irmãs, Betsabé e Sonia, pelo amor, total apoio, confiança e conselhos a mim dedicados durante toda minha vida. A meus sobrinhos Iván, Daniela e Natalia, por serem fonte de alegria e motivação.

À Universidade de São Paulo que permitiu, através do Instituto de Matemática e Estatística (IME), o meu crescimento científico.

À Profa. Claudia Garcia, pela amizade, conselhos e inúmeras discussões durante a elaboração deste trabalho.

Aos professores do IME-USP, de forma especial à Profa. Joyce Bevilacqua e ao Prof. Alexandre Roma. Aos funcionários das secretarias, biblioteca, setor de pós-graduação e sala de café que de forma direta e/ou indireta contribuíram para a realização deste trabalho.

Ao meu quase-irmão Santos, pela sua amizade, conselhos e até mesmo pelas broncas que me dava quando realmente merecia.

À Cassi e o Henrique, que me brindaram a sua amizade e uma família no Brasil.

À Larissa Sartori, pela amizade e valiosa ajuda na etapa final deste trabalho.

Agradeço aos meus amigos e colegas, pelo companheirismo, ajuda, bons momentos e por terem contribuído para que a vida aqui no Brasil ficasse mais alegre; Diane, Priscila, Catalina, Anderson, Nils, Jorge, Daniel e Marcello. 


\section{Resumo}

NAVARRETE, N. L. V. Paralelização de um modelo global de previsão do tempo em malhas localmente refinadas. 2014. 106 f. Tese (Doutorado) - Instituto de Matemática e Estatística, Universidade de São Paulo, São Paulo, 2014.

O objetivo principal deste trabalho é a paralelização de um modelo global de previsão do tempo em diferenças finitas com refinamento local. Este é baseado nas equações primitivas, e faz uso de uma discretização semi-Lagrangiana e semi-implícita em três níveis no tempo em uma malha de Lorenz na vertical e uma malha do tipo C de Arakawa na horizontal. A discretização horizontal é feita através de diferenças finitas de segunda ordem. A equação escalar elíptica tridimensional resultante é desacoplada em um sistema de equações bidimensionais do tipo Helmholtz, o qual é resolvido por meio de um método multigrid. O modelo de paralelização foi desenvolvido para máquinas com memória distribuída, fazendo uso de MPI para passagens de mensagens e baseado em técnicas de decomposição de domínio. O acoplamento apenas local dos operadores de diferenças finitas viabiliza a decomposição em duas direções horizontais. Evitamos a decomposição vertical, tendo em vista o forte acoplamento nesta direção das parametrizações de fenômenos físicos. A estratégia de paralelização foi elaborada visando o uso eficiente de centenas ou alguns milhares de processadores, dependendo da resolução do modelo. Para tal, a malha localmente refinada é separada em três regiões: uma grossa, uma de transição e uma fina, onde cada uma delas é dividida de forma independente entre um número de processadores proporcional ao número de pontos que cada uma armazena, garantindo assim um balanceamento de carga adequado. Não obstante, para resolver o sistema de equações bidimensionais do tipo Helmholtz foi necessário mudar a estratégia de paralelização, dividindo o domínio unicamente nas direções vertical e latitudinal. Ambas partes do modelo com paralelizações diferentes estão conectadas por meio da estratégia de transposição de dados. Testamos nosso modelo utilizando até 1024 processadores e os resultados ainda mostraram uma boa escalabilidade.

Palavras-chave: computação paralela, simulação numérica do tempo, refinamento local, multigrid. 


\section{Abstract}

\section{NAVARRETE, N. L. V. Parallelization of a numerical weather prediction global}

model with local refinement grids. 2014. 106 f. Tese (Doutorado) - Instituto de Matemática e Estatística, Universidade de São Paulo, São Paulo, 2014.

The main goal of this work is the parallelization of a weather prediction model employing finite differences on locally refined meshes. The model is based on the primitive equations and uses a three-time-level semi-implicit semi-Lagrangian temporal discretization on a Lorenztype vertical grid combined with a horizontal Arakawa C-grid. The horizontal discretization is performed by means of second order finite differences. The resulting three-dimensional scalar elliptic equation is decoupled into a set of Helmholtz-type two-dimensional equations, solved by a multigrid method. The parallelization has been written for distributed-memory machines, employing the MPI message passing standard and was based on domain decomposition techniques. The local coupling of the finite difference operators was exploited in a two-dimensional horizontal decomposition. We avoid a vertical decomposition due to the strong coupling of physical parameterization routines. The parallelization strategy has been designed in order to allow the efficient use of hundreds to a few thousand processors, depending on the model resolution. In order to achieve this, the locally refined mesh is split into three regions: a coarse, a transition and a fine one, each decomposed independently. The number of allocated processors for each region is proportional to the number of the grid-points it contains, in order to guarantee a good load-balancing distribution. However, to solve the set of Helmholtz-type bidimensional equations it was necessary to change the parallelization strategy, splitting the domain only in vertical and latitudinal directions. Both parts of the model with different parallelizations are related by means the data transposition strategy. We tested our model using up to 1024 processors and the results still showed a good scalability.

Keywords: parallel computing, numerical weather simulation, local refinement, multigrid. 


\section{Sumário}

$\begin{array}{ll}\text { Lista de Figuras } & \text { ix }\end{array}$

Lista de Tabelas $\quad$ xiii

$\begin{array}{ll}\text { Introdução } & 1\end{array}$

1 Formulação do modelo $\quad 7$

1.1 Equações contínuas . . . . . . . . . . . . . . . . . . . . 7

1.2 Discretização temporal . . . . . . . . . . . . . . . . . . . . . . 12

1.2 .1 Aspectos gerais . . . . . . . . . . . . . . . . . . 12

1.2 .2 Cálculo do ponto de partida . . . . . . . . . . . . . 13

1.2.3 Discretização semi-Lagrangiana . . . . . . . . . . . . . . . . . 16

1.3 Discretização vertical . . . . . . . . . . . . . . . . . . . . . . . . . . 20

1.4 Discretização horizontal . . . . . . . . . . . . . . . . . . . 27

1.5 Solução numérica . . . . . . . . . . . . . . . . . . . . 27

1.5.1 Método Multigrid . . . . . . . . . . . . . . . 27

1.5.2 Resumo de um passo no tempo . . . . . . . . . . . . . . . 28

1.5.3 Malhas com refinamento local . . . . . . . . . . . . . 29

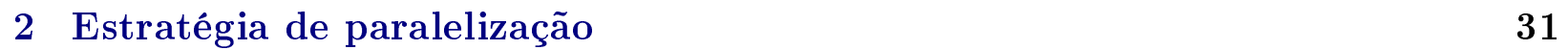

2.1 Faixas e remendos . . . . . . . . . . . . . . . . . . . . . . 35

2.2 Representação de malhas irregulares . . . . . . . . . . . . . . . . . 36

2.3 Definição dos campos . . . . . . . . . . . . . . . . . . . . . . . . . 38

2.4 Decomposição do domínio . . . . . . . . . . . . . . . . . . . . 40

2.4.1 Decomposição da malha fina . . . . . . . . . . . . . . . . 40

2.4.2 Decomposição da malha grossa . . . . . . . . . . . . . . . . 42

2.4.3 Decomposição da zona de transição . . . . . . . . . . . . . . . . . 43

2.5 Pontos inativos e comunicação . . . . . . . . . . . . . . . . . . . . . 49

2.6 Paralelização do Multigrid . . . . . . . . . . . . . . . . . . 50

2.6.1 Decomposição vertical . . . . . . . . . . . . . . . . . . 51

2.6.2 Decomposição horizontal . . . . . . . . . . . . . . . . . 51

2.7 Transposição dos dados . . . . . . . . . . . . . . . . . . . . . . . 53 
viii SUMÁRIO

3 Testes computacionais $\quad 55$

3.1 Validação do método multigrid . . . . . . . . . . . . . . . 55

3.2 Validação do modelo . . . . . . . . . . . . . . . . . 56

3.2.1 Teste em regime permanente . . . . . . . . . . . . 57

3.2 .2 Teste da onda baroclínica . . . . . . . . . . . . . . . . . . . 58

3.2.3 Teste da onda baroclínica com refinamentos locais . . . . . . . . . . . 61

3.2.4 Teste com dados reais e refinamentos locais . . . . . . . . . . . . 66

3.3 Teste de desempenho . . . . . . . . . . . . . . . . . . . 70

$\begin{array}{ll}\text { Conclusões } & 79\end{array}$

$\begin{array}{ll}\text { Referências Bibliográficas } & 81\end{array}$ 


\section{Lista de Figuras}

1.1 Diagrama ilustrando a interpolação quase-cúbica. . . . . . . . . . . . . . . 13

1.2 Vetores ao longo da trajetória. . . . . . . . . . . . . . . . . 17

1.3 Divisão da atmosfera em camadas e posição das variáveis nas mesmas. . . . . 21

1.4 Malha $\mathrm{C}$ e variáveis do modelo perto do polo. . . . . . . . . . . . . . . . 27

1.5 Malha base com dois níveis de refinamento. . . . . . . . . . . . . . . . . . 29

1.6 Malha refinada com pontos fantasmas. . . . . . . . . . . . . . . . 30

2.1 Regiões grossa, de transição e fina para a tabela dada. . . . . . . . . . . . . 32

2.2 Dimensões das malhas no arquivo de configuração. . . . . . . . . . . . . . . 33

2.3 Pontos U próximos da fronteira entre duas malhas (versão original). . . . . . 34

2.4 Pontos U próximos da fronteira entre duas malhas (versão final). . . . . . . . 35

2.5 Remendos com linhas /colunas completas. . . . . . . . . . . . . . . . 35

2.6 Remendos com quebra de linhas/colunas. . . . . . . . . . . . . . . . . . 36

2.7 Malha (logicamente) irregular. . . . . . . . . . . . . . . . . . . . . 37

2.8 Estrutura do descritor de malhas irregulares. . . . . . . . . . . . . . . 37

2.9 Descritor zonal para a Figura 2.7. . . . . . . . . . . . . . . . 37

2.10 Descritor meridional para a Figura 2.7 . . . . . . . . . . . . . . . . . 38

2.11 Varredura de uma malha irregular por segmentos. . . . . . . . . . . . . 38

2.12 Estrutura de dados para um campo tridimensional. . . . . . . . . . . . . . 39

2.13 Varredura de um conjunto de malhas irregulares por segmentos. . . . . . . . 39

2.14 Divisão de uma malha irregular em faixas e conteúdo do mapa $(\mathrm{X}<0)$. . . . 42

2.15 Dados para uma malha grossa com 4 refinamentos locais. . . . . . . . . . . 44

2.16 Decomposição da malha grossa para os dados da Tabela 2.1. . . . . . . . . . 44

2.17 Decomposição da malha fina para os dados da Tabela 2.1. . . . . . . . . . . 45

2.18 Decomposição da malha grossa para os dados da Tabela 2.2. . . . . . . . . . 45

2.19 Decomposição da malha fina para os dados da Tabela 2.2. . . . . . . . . . . 46

2.20 Decomposição das malhas para os dados da Tabela 2.2 . . . . . . . . . . . 46

2.21 Decomposição da malha grossa para os dados da Tabela 2.3. . . . . . . . . . . 47

2.22 Decomposição da malha grossa para os dados da Tabela 2.3, utilizando a técnica IGLOO . . . . . . . . . . . . . . . . . . . . 47

2.23 Projeção estereográfica da malha grossa, para a Tabela 2.3 . . . . . . . . . . 48 
2.24 Projeção estereográfica da malha grossa para a Tabela 2.3, utilizando a técnica IGLOO.

3.1 Configuração de malhas para o teste de convergência do método multigrid.

3.2 Norma $l_{2}(\mathrm{~m} / \mathrm{s})$ da perda de simetria do vento zonal em relação à sua média zonal.

3.3 Norma $l_{2}(\mathrm{~m} / \mathrm{s})$ da degradação da média zonal do vento zonal em relação à solução exata.

3.4 Evolução da pressão de superfície (esquerda) e da temperatura em $850 \mathrm{hPa}$ (direita) para uma malha com resolução horizontal de $1024 \times 513$ pontos, 26 níveis verticais e $\Delta t=450 \mathrm{~s} . \ldots \ldots \ldots \ldots \ldots$

3.5 Pressão de superfície (esquerda) e temperatura em $850 \mathrm{hPa}$ (direita) após 9

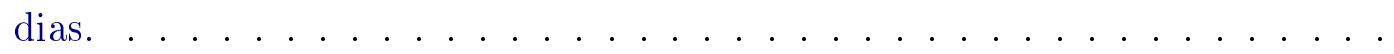

3.6 Pressão de superfície (esquerda) e temperatura em $850 \mathrm{hPa}$ (direita) após 10 dias.

3.7 Norma $l_{2}(\mathrm{hPa})$ da variação da pressão de superfície ao longo do tempo para varias resoluções, em relação à resolução de referência $1024 \times 513 \times 26$. . . .

3.8 Norma $l_{2}\left({ }^{\circ} \mathrm{K}\right)$ da variação da temperatura, no nível $\sigma=0.865$, ao longo do tempo para varias resoluções, em relação à resolução de referência $1024 \times 513 \times 26$. 62

3.9 Configuração das malhas para o teste da onda baroclínica com refinamentos locais. . . . . . . . . . . . . . . . . .

3.10 Localizaçao dos refinamentos locais e pressão de superfície inicial para o teste da onda baroclínica com refinamentos locais. . . . . . . . . . . . . .

3.11 Pressão de superfície (esquerda) e temperatura em $850 \mathrm{hPa}$ (direita) para o teste da onda baroclínica. Em cores os resultados do refinamento local mais fino e em preto os da malha de referência.

3.12 Norma $l_{2}(\mathrm{hPa})$ sobre a área do refinamento local mais fino, da diferença da pressão de superfície entre a solução de referencia na malha uniforme e a solução calculada empregando refinamento local . . . . . . . . . . . . . .

3.13 Norma $l_{2}\left({ }^{\circ} \mathrm{K}\right)$ sobre a área do refinamento local mais fino, da diferença da temperatura entre a solução de referencia na malha uniforme e a solução calculada empregando refinamento local . . . . . . . . . . . . . . 65

3.14 Configuração das malhas para o teste com dados reais e refinamentos locais.

3.15 Localizaçao dos refinamentos locais e pressão de superfície inicial para o teste com dados reais e refinamentos locais. . . . . . . . . . . . . . . . . . . .

3.16 Pressão de superfície (esquerda) e altura geopotencial em 500hPa (direita) para o teste com dados reais. Em cores os resultados do refinamento local mais fino e em preto os da malha de referência.

3.17 Norma $l_{2}(\mathrm{hPa})$ da diferença da pressão de superfície entre o refinamento local mais fino e a resolução de referência $1024 \times 513 \times 26$, ao longo do tempo. . . 
3.18 Norma $l_{2}\left({ }^{\circ} \mathrm{K}\right)$ da diferença da temperatura entre o refinamento local mais fino e a resolução de referência $1024 \times 513 \times 26$, ao longo do tempo. . . . . . 69

3.19 Configuração da malha base e 4 refinamentos locais utilizadas no teste de desempenho. . . . . . . . . . . . . . . . . 71

3.20 Speedup obtido pela bateria de testes no cluster tupã . . . . . . . . . . . . 72

3.21 Eficiência obtida pela bateria de testes no cluster tupã . . . . . . . . . . . 73

3.22 Tempos mínimos, médios e máximos das principais etapas ocorridas durante a integração do modelo, para o teste de desempenho. . . . . . . . . . . . 74

3.23 Tempos mínimos, médios e máximos, segundo a região e total, gastos no transporte Semi-Lagrangiano, para o teste de desempenho com 1024 processadores

3.24 Tempos mínimos, médios e máximos para os processadores da malha fina, separados em interiores, de fronteira e valores totais. . . . . . . . . . . . 75

3.25 Tempos total, de comunicação, de cómputo em pontos inativos e de cómputo em pontos ativos para o transporte Semi-Lagrangiano, para 1024 processadores. 76

3.26 Porcentagem dos tempos mínimos, médios e máximos, relativo ao tempo total médio, utilizado na transposição dos dados, para o teste de desempenho. 77 


\section{Lista de Tabelas}

2.1 Pontos por processador para cada região e para o caso ideal, para 20 processadores. . . . . . . . . . . . . . . . . . . . 44

2.2 Pontos por processador para cada região e para o caso ideal, para 79 processadores. . . . . . . . . . . . . . . . . . . . 45

2.3 Pontos por processador para cada região e para o caso ideal, para 237 processadores. . . . . . . . . . . . . . . . . . . . . 47

3.1 Fatores de convergência do multigrid para a equação (1.56) e os três maiores

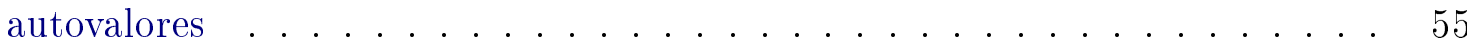

3.2 Fatores de convergência do multigrid para a equação (1.56) em malhas localmente refinadas. . . . . . . . . . . . . . . . . . 56

3.3 Distribuição de processadores por região . . . . . . . . . . . . . . . . 71

3.4 Tempos totais dos testes realizados no cluster tupã $\ldots \ldots \ldots$. . . . . . . 72 


\section{Introdução}

O tempo e o clima influenciam profundamente a economia e a infraestrutura da sociedade, desde o abastecimento de alimentos aos recursos energéticos ou às atividades de lazer, motivo pelo qual a previsão do tempo e clima é de vital importância para a mesma. A previsão numérica do tempo consiste em utilizar as condições ambientais atuais (temperatura, precipitação, etc.) para produzir, com o auxílio do computador, condições prováveis em um determinado tempo e lugar. Quanto mais refinados são os dados, mais precisa é a previsão, o que significa que os requisitos computacionais e de armazenamento de dados da previsão numérica do tempo são muito altos. Desde o inicio da ciência da computação, os cientistas que trabalham nesta área tem sempre sacado proveito dos últimos avanços na tecnologia da computação.

Para produzir uma previsão numérica do tempo é necessário modelar a dinâmica da atmosfera, resolvendo um conjunto de equações diferenciais parciais munido de condições iniciais e de fronteira, e dos fenômenos físicos que ocorrem nela, normalmente por meio de esquemas de parametrização de sub-escala. Sua eficiência e veracidade dependem diretamente da resolução temporal e espacial da malha trabalhada. Desta forma, a computação paralela é necessária para reduzir o tempo computacional e para distribuir o problema, de forma que os dados possam ser contidos na memória.

Atualmente os computadores paralelos mais comuns e mais potentes são máquinas do tipo MIMD (Multiple Instruction stream, Multiple Data stream) ${ }^{1}$ e para a aplicação a ser resolvida neste trabalho o estilo de programação usada será SPMD (Single Program, Multiple Data). Assim, cada processador executa o mesmo programa assincronamente e o problema é resolvido simultaneamente dividindo os dados em diferentes partes sobre as quais os processadores trabalham.

Executar um modelo global em alta resolução pode ser extremamente custoso ou até mesmo inviável, motivo pelo qual os centros de previsão do tempo têm tradicionalmente utilizado dois modelos operacionais para realizar suas previsões; um global e um regional. Desta forma, o modelo global é executado em uma resolução baixa (malha grossa) e uniforme com o objetivo de fornecer uma previsão de médio prazo e de gerar condições iniciais e de fronteira para o modelo regional (malha fina), o qual é executado com uma resolução uniforme maior para produzir uma previsão a curto prazo e mais detalhada em uma região

\footnotetext{
${ }^{1}$ http://www.top500.org/
} 
de interesse específica. Uma caraterística destes modelos é a interação em uma via, do modelo global ao regional, já que a informação na malha fina não afeta a solução no modelo global. Empregar esta metodologia causa certos problemas, pois a esfera não tem fronteiras e o modelo regional precisa dessas condições que são artificiais. Por outro lado, o modelo global pode fornecer estas condições, mas o modelo regional não reenvia suas informações para o global.

As condições de fronteira são essencialmente o problema desta metodologia, pois não são naturais para o problema e que obriga, no modelo regional, a utilização de diversas técnicas empíricas, tal como suavização em regiões próximas à fronteira para minimizar os efeitos da prescrição dos campos meteorológicos na fronteira.

Vários centros de meteorologia utilizam esta metodologia. O modelo global dos Centros Nacionais de Previsão Ambiental (NCEP, EUA), chamado de GFS [3], é um modelo hidrostático, Euleriano, espectral e com malha horizontal gaussiana enquanto o modelo regional WRF [38] é não hidrostático, em diferenças finitas e com malha horizontal lat-lon do tipo E de Arakawa.

No caso do Serviço Meteorológico Alemão (DWD) o modelo espectral, chamado de GME [34], é um modelo hidrostático, Euleriano, em diferenças finitas e malha horizontal icosaédrica-hexagonal, que pode ser considerada como a união (lógica) de dez losangos e no processo de paralelização, feito no sentido horizontal, cada processador ocupa-se de um subdomínio dos dez losangos. Este modelo fornece com condições iniciais e de fronteira o modelo regional COSMO [2] (COSMO-EU para Europa e COSMO-DE para Alemanha), que é um modelo não hidrostático, com esquema de divisão explícito, em diferenças finitas e com malha horizontal lat-lon do tipo C de Arakawa. No entanto, o projeto ICON [4], modelo não hidrostático com malha icosaédrica e refinamento local, visa substituir ambos os modelos GME e COSMO.

O Centro de Previsão de Tempo e Estudos Climáticos (CPTEC, Brasil) possui um modelo global, chamado de MGCA [6], hidrostático, Euleriano ou semi-Lagrangiano, espectral e com malha horizontal Gaussiana. Este modelo alimenta o modelo regional BRAMS [1] com condições iniciais e de fronteira, que por sua vez é um modelo não hidrostático, em diferenças finitas e malha horizontal lat-lon do tipo C de Arakawa.

Entretanto, empregar dois modelos, global e regional, implica a manutenção, atualização e otimização de dois conjuntos de bibliotecas e procedimentos. Como alternativa para a metodologia acima descrita e com o objetivo de eliminar as condições de fronteira artificiais e de se obter um modelo integrado (no sentido de unificar os modelos globais e regionais), têm surgido os modelos com resolução variável (um resumo das principais técnicas deste tipo pode ser encontrado em [22]). Uma caraterística destes modelos é a possibilidade de se ter uma interação de duas vias, pois neste caso os valores da fronteira do refinamento local são fornecidos pela malha grossa, ao passo que os valores na malha grossa podem ser atualizados pelos valores no interior do refinamento. Como outros fatores importantes para o uso de modelos com resolução variável podemos mencionar a necessidade de se manter a 
precisão de uma simulação conforme se desenvolvem as características de menor escala ou a necessidade de aumentar a resolução sobre uma determinada área de interesse mantendo ao mesmo tempo a sua fronteira o suficientemente afastada com o objetivo de que não influencie desfavoravelmente a previsão sobre dita área. Ainda mais, utilizar um modelo unificado tem a vantagem de que somente é necessário uma equipe trabalhando e desenvolvendo o mesmo modelo, acarretando consequências positivas na manutenção, documentação e uso do código. Um outro aspecto importante relacionado ao modelo unificado é a possibilidade de se ter um único sistema de assimilação de dados.

O uso da resolução variável pode ser dividido em duas classes: o sistema de malhas permanece fixo durante a integração inteira ou se adapta continuamente em resposta ao tamanho do erro local ou em relação a um campo particular de interesse. Ainda mais, o sistema de malhas pode ser estruturado ou não estruturado. Uma família de modelos com resolução variável e cujo sistema de malhas permanece fixo durante a integração é aquela que introduz uma transformação de variáveis de modo que uma resolução uniforme no domínio transformado implica uma resolução variável no domínio físico. Desta forma uma estratégia computacional de resolução uniforme pode ser aplicada às equações transformadas.

Entre os modelos que utilizam esta metodologia, podemos mencionar o do Centro Meteorológico Canadense (CMC), cujo modelo unificado, chamado GME [24], é não hidrostático, em diferenças finitas e com malha horizontal lat-lon do tipo C de Arakawa, que pode ser arbitrariamente rotada para enfocar qualquer parte da esfera com maior resolução aplicando uma transformação [23] (malha tilt and stretched). O paralelismo é feito bidimensionalmente no sentido horizontal, mantendo intacta a direção vertical [51].

Um outro modelo deste tipo é o modelo unificado do Serviço Meteorológico Nacional do Reino Unido (Met Office), descrito em [25], [48] e [49]. Este modelo é não-hidrostático, em diferenças finitas e malha horizontal lat-lon do tipo $\mathrm{C}$ de Arakawa com resolução variável (tilt and stretched).

Recentemente, em julho de 2014, entrou em operação no Met Office o núcleo dinâmico ENDGame [35]. Como no modelo anterior, consiste em um modelo não hidrostático, em diferenças finitas e malha horizontal lat-lon do tipo C de Arakawa. Entretanto, o ENDGame tem sido projetado para considerar diversas opções, que podem ser ativadas ou não, tais como ser hidrostático ou não hidrostático, considerar uma atmosfera rasa ou profunda e utilizar coordenadas cartesianas, esféricas ou esferoidais. Espera-se que o ENDGame permaneça em operação até o ano 2022, aproximadamente, quando deverá ser substituído pelo Gung Ho [26], novo núcleo dinâmico do Met Office e ainda em fase de projeto. Entre as principais características que Gung Ho deverá ter estão a remoção da singularidade nos polos, a escalabilidade até centenas de milhares de processadores e a conservação inerente do esquema de advecção.

O modelo unificado do Serviço Meteorológico Francês (Météo-France), chamado de ARPEGE e descrito em [56], é um modelo não hidrostático, spectral e malha horizontal gaussiana com projeção Schmidt que lhe permite ter uma resolução variável. Segundo [24], devido a 
limitações da transformada de Schmidt na obtenção de resolução variável com modelos espectrais, foi necessário desenvolver uma versão de área limitada de ARPEGE, chamada de ALADIN, sendo não hidrostático e ainda espectral, pelo que é bi-periódico. A resolução horizontal deste modelo é de aproximadamente $7 \mathrm{Km}$. Adicionalmente foi desenvolvido o modelo AROME, de área limitada, para altas resoluções horizontais da ordem de $1 \mathrm{Km}$ a $2.5 \mathrm{Km}$. A diferença principal entre AROME e ALADIN é o pacote das parametrizações físicas.

Um outro tipo de modelo com resolução variável é o modelo global com malhas localmente refinadas. Neste tipo de modelo, a partir de uma malha global com resolução uniforme são inseridas (e mantidas fixas) malhas gradativamente mais finas em torno de uma região de interesse, mas sem estas estarem sobrepostas. Uma característica deste sistema de malhas é que duas malhas adjacentes podem ser acopladas dinamicamente quando a integração no tempo para os pontos próximos da interface é realizado, em cada lado, utilizando a informação da outra malha. Modelos deste tipo, dentro do contexto das equações de água rasa, tem sido propostos em [28] e [15] e, recentemente em [17], tem sido proposto um modelo hidrostático, em diferenças finitas e malha horizontal lat-lon do tipo C de Arakawa.

A técnica de malhas dinamicamente refinadas (AMR), desenvolvida em [20] e [19], também tem sido usada em modelos de previsão do tempo. Estes modelos caraterizam-se em que as malhas não são refinadas de forma antecipada e sim durante a sua integração, onde e quando é necessária uma maior resolução. Da mesma forma, se em alguma região refinada não é mais necessária uma resolução fina, a malha é engrossada. Desta forma, são necessários critérios para determinar onde e quando as malhas devem ser refinadas/engrossadas. Em [46] é estudada a advecção de um ciclone barotrópico e o desenvolvimento de uma perturbação baroclínica, onde a criação/remoção dos refinamentos locais é controlada durante a execução por meio de uma estimativa do erro de truncamento. Em [45] é apresentado um modelo de mesoescala não hidrostático onde a resolução é seletivamente ampliada em diversas regiões utilizando AMR, na simulação de uma super célula de tempestade. Em [31] é apresentado um modelo hidrostático, em volumes finitos e com malhas horizontal estruturada por blocos e cuja resolução pode-se adaptar de forma estática ou dinâmica. Já em [10] é descrito o modelo operacional OMEGA, modelo não hidrostático em volumes finitos e com malha horizontal não estruturada triangular.

Em [16] é desenvolvido um modelo global hidrostático, semi-implícito e semi-Lagrangiano, em diferenças finitas, com malha horizontal uniforme lat-lon do tipo C da Arakawa e coordenada vertical $\sigma$. Este trabalho propõe a paralelização deste modelo, para máquinas com memória distribuída e utilizando passagem de mensagens, acrescentando-lhe malhas localmente refinadas, como descrito em [28] e [15], com o objetivo de termos um modelo com resolução variável. A seguir, no Capítulo 1, descrevemos a formulação do modelo e o sistema de malhas localmente refinadas. No Capítulo 2, parte central deste trabalho, apresentamos a estratégia de paralelização do modelo que, devido ao acoplamento local das diferenças finitas e ao acoplamento vertical das parametrizações físicas, foi realizada decompondo horizontalmente o domínio, mantendo intacta a direção vertical. Ainda mais, com o intuito de 
minimizar a comunicação entre os processadores, modificamos convenientemente a definição do sistema de malhas. Todavia, para resolver o sistema de equações bidimensionais do tipo Helmholtz que resulta da discretização semi-implícita, empregando o método multigrid, foi necessário mudar o sentido da paralelização, decompondo o domínio nas direções vertical e latitudinal e mantendo intacta a direção longitudinal. A conexão entre ambas as partes do modelo com paralelizações diferentes é realizada empregando a técnica de transposição de dados [14]. No Capítulo 3 apresentamos e discutimos os nossos testes computacionais. Validamos primeiro o método multigrid resolvendo uma das equações do sistema de equações bidimensionais do tipo Helmholtz, com o lado direito compelido de forma que a solução exata seja conhecida. Logo depois validamos o modelo utilizando o teste proposto em [33] e em seguida utilizando dados reais. O último teste consiste em uma avaliação de desempenho do nosso modelo no cluster tupã do CPTEC, onde foi executado utilizando até 1024 processadores. Por último, apresentamos as conclusões deste trabalho. 
INTRODUÇÃO 


\section{Capítulo 1}

\section{Formulação do modelo}

Este capítulo descreve o modelo global de previsão do tempo em três níveis no tempo, semi-implícito, semi-Lagrangiano e em diferenças finitas, cujo desenvolvimento pode ser encontrado em [16]. Outros modelos do mesmo tipo podem ser vistos em [18] e [37]. Também descrevemos a metodologia desenvolvida em [28] e em [15] para a eficiente utilização de malhas com refinamento local.

\subsection{Equações contínuas}

As equações primitivas, conjunto de equações diferenciais parciais não lineares utilizadas para aproximar o estado da atmosfera, constituem o núcleo da maioria dos modelos globais de previsão do tempo e são dadas por (veja [29] e [30]):

equação do momento horizontal

$$
\left(\frac{d \mathbf{V}}{d t}\right)_{H}=-\nabla \Phi-R T \nabla P-f \mathbf{k} \times \mathbf{V},
$$

equação da termodinâmica

$$
\frac{d T}{d t}=\kappa T\left(\frac{\dot{\sigma}}{\sigma}+\frac{d P}{d t}\right)
$$

equação da continuidade

$$
\frac{d P}{d t}=-\nabla \cdot \mathbf{V}-\frac{\partial \dot{\sigma}}{\partial \sigma}
$$

em que adotamos $\sigma=\frac{p}{p_{s}}$ como variável vertical. completar estas equações a Completando estas equações temos a

equação hidrostática

$$
\sigma \frac{\partial \Phi}{\partial \sigma}=-R T
$$

acompanhada das condições de fronteira

$$
\dot{\sigma}=0, \quad \text { em } \quad \sigma=0 \text { e } \sigma=1,
$$


sendo que,

$$
\begin{aligned}
p & =\text { pressão } \\
p_{s} & =\text { pressão de superfície } \\
\mathrm{t} & =\text { tempo } \\
\mathbf{V} & =\text { velocidade horizontal }(=u \mathbf{i}+v \mathbf{j}) \\
\frac{d}{d t} & =\text { derivada total tridimensional } \\
\left(\frac{d \mathbf{V}}{d t}\right)^{H} & =\text { projeção horizontal de } \frac{d \mathbf{V}}{d t} \\
\nabla & =\text { operador gradiente horizontal } \\
\lambda & =\text { longitude } \\
\theta & =\text { latitude } \\
a & =\text { raio da Terra } \\
\Phi & =\text { geopotencial } \\
R & =\text { constante dos gases } \\
T & =\text { temperatura } \\
P & =\text { ln } p_{s} \\
f & =\text { força de Coriolis } \\
\mathbf{k} & =\text { vetor unitário na vertical } \\
\dot{\sigma} & =\text { velocidade vertical } \\
\kappa & =\frac{R}{C_{p}} \\
C_{p} & =\text { calor especifico do ar a pressão constante }
\end{aligned}
$$

Uma primeira observação é o fato de $P$ ser independente da variável vertical $\sigma$, e portanto temos a seguinte simplificação para a equação da continuidade (1.3):

$$
\frac{d P}{d t}=\frac{\partial P}{\partial t}+\mathbf{V} \cdot \nabla P+\dot{\sigma} \frac{\partial P}{\partial \sigma}=\frac{\partial P}{\partial t}+\mathbf{V} \cdot \nabla P
$$

\section{Equação do momento}

Para a equação do momento, consideramos a temperatura $T$ como a soma de uma temperatura média constante $T_{0}$ com uma perturbação $T^{\prime}$, assim podemos reescrever a equação (1.1) da seguinte forma

$$
\begin{aligned}
\left(\frac{d \mathbf{V}}{d t}\right)_{H} & =-\nabla \Phi-R\left(T_{0}+T^{\prime}\right) \boldsymbol{\nabla} P-f \mathbf{k} \times \mathbf{V} \\
& =-\nabla \Phi-R T_{0} \boldsymbol{\nabla} P-R T^{\prime} \nabla P-f \mathbf{k} \times \mathbf{V} \\
& =-\boldsymbol{\nabla}\left(\Phi+R T_{0} P\right)-f \mathbf{k}-\times \mathbf{V}-R T^{\prime} \nabla P \\
& =-\nabla G-f \mathbf{k} \times \mathbf{V}-R T^{\prime} \nabla P,
\end{aligned}
$$

em que denominamos $G=\Phi+R T_{0} P$ de geopotencial generalizado. 


\section{Equação hidrostática}

Para esta equação vamos utilizar duas formas. A primeira delas é obtida integrando a equação (1.4) na coluna vertical

$$
\int_{0}^{1} \sigma \frac{\partial \Phi}{\partial \sigma} d \sigma=-\int_{0}^{1} R T d \sigma
$$

considerando que

$$
\frac{\partial}{\partial \sigma}(\sigma \Phi)=\Phi+\sigma \frac{\partial \Phi}{\partial \sigma}
$$

temos

$$
-\int_{0}^{1} \Phi d \sigma+\int_{0}^{1} \frac{\partial}{\partial \sigma}(\sigma \Phi) d \sigma=-R \int_{0}^{1} T d \sigma
$$

ou

$$
\int_{0}^{1} \Phi d \sigma=\Phi_{s}+R \int_{0}^{1} T d \sigma
$$

o qual denominamos $\Phi_{s}=\Phi(\lambda, \theta, 1)$ de geopotencial orográfico, sendo o seu valor igual ao produto da gravidade pela altura da topografia terrestre. A segunda forma da equação hidrostática é dada pela fórmula

$$
\frac{\partial \Phi}{\partial \sigma}+C_{p} \Theta \frac{\partial \Pi}{\partial \sigma}
$$

em que $\Pi=\left(\frac{p}{p_{0}}\right)^{\kappa}$ é a pressão normalizada, com $p_{0}$ constante e $\Theta$ é a temperatura virtual satisfazendo a relação $T=\Pi \Theta$.

\section{Equação da continuidade}

Para a equação da continuidade, também utilizaremos duas formas alternativas, sendo a primeira delas obtida ao integrar a equação (1.3) na coluna vertical

$$
\int_{0}^{1}\left(\frac{\partial P}{\partial t}+\mathbf{V} \cdot \nabla P\right) d \sigma=-\int_{0}^{1}\left(\nabla \cdot \mathbf{V}+\frac{\partial \dot{\sigma}}{\partial \sigma}\right) d \sigma
$$

Aplicando as condições de fronteira (1.5) e observando que $P$ independe da variável vertical $\sigma$, obtemos

$$
\frac{\partial P}{\partial t}+\int_{0}^{1} \mathbf{V} \cdot \nabla P d \sigma=-\int_{0}^{1} \nabla \cdot \mathbf{V} d \sigma .
$$

Do segundo termo do lado esquerdo da equação acima, segue que 


$$
\begin{aligned}
\int_{0}^{1} \mathbf{V} \cdot \nabla P d \sigma & =\int_{0}^{1}\left(\sum_{i} v_{i} \frac{\partial P}{\partial x_{i}}\right) d \sigma \\
& =\sum_{i} \int_{0}^{1} v_{i} \frac{\partial P}{\partial x_{i}} d \sigma \\
& =\sum_{i} \frac{\partial P}{\partial x_{i}} \int_{0}^{1} v_{i} d \sigma \\
& =\nabla P \cdot \int_{0}^{1} \mathbf{V} d \sigma
\end{aligned}
$$

Assim, definindo a velocidade média (na coluna vertical)

$$
\overline{\mathbf{V}}=\int_{0}^{1} \mathbf{V} d \sigma
$$

podemos definir a derivada total bidimensional

$$
\left(\frac{d}{d t}\right)_{2}=\frac{\partial}{\partial t}+\overline{\mathbf{V}} \cdot \nabla
$$

com o qual a equação (1.9) torna-se

$$
\left(\frac{d P}{d t}\right)_{2}=-\int_{0}^{1} \nabla \cdot \overline{\mathbf{V}} d \sigma
$$

Com o intuito de diminuir possíveis respostas ressonantes falsas às forçantes orográficas, separamos $P$ da forma

$$
P=l^{\prime}-\frac{\Phi_{s}}{R T_{0}}
$$

que é uma adaptação da proposta feita em [43]. Desta forma, a equação (1.10) será dada por

$$
\left(\frac{d l^{\prime}}{d t}\right)_{2}-\left(\frac{d}{d t}\left(\frac{\Phi_{s}}{R T_{0}}\right)\right)_{2}=-\int_{0}^{1} \nabla \cdot \mathbf{V} d \sigma
$$

$\mathrm{Ou}$

$$
\left(\frac{d l^{\prime}}{d t}\right)_{2}=-\int_{0}^{1} \nabla \cdot \mathbf{V} d \sigma+\frac{1}{R T_{0}} \overline{\mathbf{V}} \cdot \nabla \Phi_{s} .
$$

A outra forma da equação da continuidade é obtida ao integrarmos a equação (1.3) desde um nível arbitrário até a superfície, isto é, 


$$
\begin{aligned}
\int_{\sigma}^{1}\left(\frac{d P}{d t}+\nabla \cdot \mathbf{V}+\frac{\partial \dot{\sigma}}{\partial \sigma}\right) d \sigma & =\int_{\sigma}^{1}\left(\frac{\partial P}{\partial t}+\mathbf{V} \cdot \nabla P+\nabla \cdot \mathbf{V}+\frac{\partial \dot{\sigma}}{\partial \sigma}\right) d \sigma \\
& =(1-\sigma) \frac{d P}{d t}+\int_{\sigma}^{1}(\mathbf{V} \cdot \nabla P+\nabla \cdot \mathbf{V}) d \sigma-\dot{\sigma} \\
& =0
\end{aligned}
$$

ou

$$
\dot{\sigma}=(1-\sigma) \frac{d P}{d t}+\int_{\sigma}^{1}(\mathbf{V} \cdot \nabla P+\nabla \cdot \mathbf{V}) d \sigma .
$$

Substituindo a equação (1.9) na equação acima, obtemos a equação para a velocidade vertical

$$
\dot{\sigma}=-(1-\sigma) \int_{0}^{1}(\mathbf{V} \cdot \nabla P+\nabla \cdot \mathbf{V}) d \sigma+\int_{\sigma}^{1}(\mathbf{V} \cdot \nabla P+\nabla \cdot \mathbf{V}) d \sigma
$$

\section{Equação da termodinâmica}

Das equações (1.2), (1.9) e (1.13), temos que

$$
\begin{aligned}
\frac{d T}{d t}= & \kappa T \frac{\dot{\sigma}}{\sigma}+\kappa T \frac{d P}{d t} \\
= & \kappa T_{0} \frac{\dot{\sigma}}{\sigma}+\kappa T^{\prime} \frac{\dot{\sigma}}{\sigma}+\kappa T\left(\frac{\partial P}{\partial t}+\mathbf{V} \cdot \nabla P\right) \\
= & \frac{\kappa T_{0}}{\sigma}\left(-(1-\sigma) \int_{0}^{1}(\mathbf{V} \cdot \nabla P+\nabla \cdot \mathbf{V}) d \sigma+\int_{\sigma}^{1}(\mathbf{V} \cdot \nabla P+\nabla \cdot \mathbf{V}) d \sigma\right) \\
& +\kappa T^{\prime} \frac{\dot{\sigma}}{\sigma}+\kappa T\left(-\int_{0}^{1}(\mathbf{V} \cdot \nabla P+\nabla \cdot \mathbf{V}) d \sigma+\mathbf{V} \cdot \nabla P\right)
\end{aligned}
$$

$\mathrm{ou}$

$$
\begin{aligned}
\frac{d T}{d t}= & \frac{\kappa T_{0}}{\sigma}\left(-(1-\sigma) \int_{0}^{1} \nabla \cdot \mathbf{V} d \sigma+\int_{\sigma}^{1} \nabla \cdot \mathbf{V} d \sigma\right)-\kappa T_{0} \int_{0}^{1} \nabla \cdot \mathbf{V} d \sigma \\
& +\kappa T^{\prime}\left(\frac{\dot{\sigma}}{\sigma}-\int_{0}^{1} \nabla \cdot \mathbf{V} d \sigma\right)+\kappa T\left(-\int_{0}^{1} \mathbf{V} \cdot \nabla P d \sigma+\mathbf{V} \cdot \nabla P\right) \\
& +\frac{\kappa T_{0}}{\sigma}\left(-(1-\sigma) \int_{0}^{1} \mathbf{V} \cdot \nabla P d \sigma+\int_{\sigma}^{1} \mathbf{V} \cdot \nabla P d \sigma\right)
\end{aligned}
$$




\subsection{Discretização temporal}

\subsubsection{Aspectos gerais}

Consideremos a equação prognóstica

$$
\frac{d F}{d t}=\Psi_{F}
$$

Podemos integrar a equação acima entre os tempos $t^{n-1}$ e $t^{n+1}$ (três níveis no tempo) pelo método semi-Lagrangiano [47], isto é, seguindo a trajetória da partícula que chega ao ponto de malha $\mathbf{x}_{\mathbf{a}}$ no tempo $t^{n+1}$. Chamamos o ponto de malha $\mathbf{x}_{\mathbf{a}}$ de ponto de chegada e a mudança no valor de $F$ da partícula que chega ao ponto $\mathbf{x}_{\mathbf{a}}$ no tempo $t^{n+1}$ é a integral de $\Psi_{F}$ seguindo a trajetória sobre o correspondente intervalo de tempo:

$$
F_{\mathrm{a}}^{n+1}-F_{\mathrm{d}}^{n-1}=\int_{t^{n-1}}^{t^{n+1}} \Psi_{F} d t=\bar{\Psi}_{F} \Delta t
$$

em que

$$
F_{\mathbf{a}}^{n+1} \equiv F\left(\mathbf{x}_{\mathbf{a}}, t^{n+1}\right) \quad \text { e } \quad F_{\mathbf{d}}^{n-1} \equiv F\left(\mathbf{x}_{\mathbf{d}}, t^{n-1}\right)
$$

são os valores de $F$ da partícula que partiu desde o ponto $\mathbf{x}_{\mathbf{d}}$ (não necessariamente um ponto de malha e que chamamos de ponto de partida) no tempo $t^{n-1}$ e chegou ao ponto de malha $\mathbf{x}_{\mathbf{a}}$ no tempo $t^{n+1}$. Observamos que são necessários métodos de interpolação para calcular os valores de $\mathbf{x}_{\mathbf{d}}$ e $F_{\mathbf{d}}$ a partir da informação disponível nos pontos de malha.

O termo $\bar{\Psi}_{F}$ na equação (1.16) é uma média no tempo de $\Psi_{F}$ ao longo da trajetória do ponto $\mathbf{x}_{\mathbf{d}}\left(\right.$ em $\left.t^{n-1}\right)$ ao ponto $\mathbf{x}_{\mathbf{a}}\left(e m t^{n+1}\right)$. Da mesma forma que para $\mathbf{x}_{\mathbf{d}}$ e $F_{\mathbf{d}}$, precisamos utilizar métodos de interpolação para calcular $\bar{\Psi}_{F}$ a partir da informação disponível nos pontos de malha. Para aproximar $\bar{\Psi}_{F}$, separamos $\Psi_{F}$ nas suas partes linear e não linear. Tratamos a parte linear $\mathcal{L}_{F}$ de forma semi-implícita, isto é, através de uma média entre valores conhecidos e desconhecidos, enquanto a parte não linear $\mathcal{N}_{F}$ é aproximada pela média entre os valores nos pontos partida e chegada, no tempo $t^{n}$. Assim, a equação (1.16) fica discretizada como

$$
F_{\mathbf{a}}^{n+1}-F_{\mathbf{d}}^{n-1}=\tau\left(\mathcal{L}_{F, \mathbf{a}}^{n+1}+\mathcal{L}_{F, \mathbf{d}}^{n-1}\right)+\tau\left(\mathcal{N}_{F, \mathbf{a}}^{n}+\mathcal{N}_{F, \mathbf{d}}^{n}\right),
$$

ou, em uma melhor notação

$$
\left(F-\tau \mathcal{L}_{F}\right)_{\mathbf{a}}^{n+1}=\tau \mathcal{N}_{F, \mathbf{a}}^{n}+\left[F^{n-1}+\tau\left(\mathcal{L}_{F}^{n-1}+\mathcal{N}_{F}^{n}\right)\right]_{\mathbf{d}},
$$

em que $\tau=\Delta t$. Observe que as equações (1.6), (1.12) e (1.14) podem ser expressadas sob a forma acima descrita.

Para calcular o ponto de partida $\mathbf{x}_{\mathbf{d}}$ utilizamos interpolação linear. Já para o cálculo dos valores de $F_{\mathbf{d}}$ e das partes linear $\left(\mathcal{L}_{F}\right)$ e não linear $\left(\mathcal{N}_{F}\right)$ de $\Psi_{F}$, utilizamos interpolação quase- 
cúbica, que é uma versão menos custosa da interpolação cúbica [44]. Ilustramos este método para duas dimensões e para uma malha uniforme na Figura 1.1, onde o ponto a ser calculado é $\left(x_{i}+\alpha, y_{j}+\beta\right)$. No primeiro passo fazemos quatro interpolações na direção $x$ : interpolação linear (em vez de cúbica) para os pontos $\left(x_{i}+\alpha, y_{j-1}\right)$ e $\left(x_{i}+\alpha, y_{j+2}\right)$, e interpolação cúbica para os pontos $\left(x_{i}+\alpha, y_{j}\right)$ e $\left(x_{i}+\alpha, y_{j+1}\right)$. No segundo passo fazemos uma interpolação cúbica na direção $y$, afim de calcular o valor do campo no ponto desejado. Desta forma, o número de pontos "vizinhos" que contribuem ao resultado reduz-se de 16 para 12. A generalização para o caso tridimensional é imediata resultando em uma redução de 64 para 32 pontos e o número de interpolações necessárias cai de 21 interpolações cúbicas unidimensionais para 7 interpolações cúbicas mais 10 interpolações lineares, todas unidimensionais.

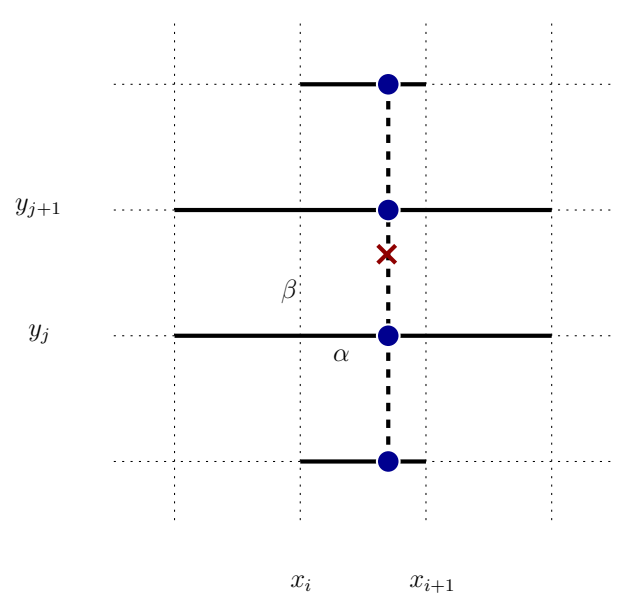

Figura 1.1: Diagrama ilustrando a interpolação quase-cúbica.

\subsubsection{Cálculo do ponto de partida}

Para calcular o ponto de partida $\mathbf{x}_{\mathbf{d}}$ calculamos primeiro o ponto médio da trajetória $\mathbf{x}_{\mathbf{m}}$, isto é feito utilizando o método desenvolvido em [40] e generalizado em [42]. Consideremos a equação

$$
\mathbf{r}_{\mathbf{a}}^{n+1}=\mathbf{r}_{\mathbf{m}}^{n}+\tau \mathbf{U}\left(\mathbf{r}_{\mathbf{m}}^{n}, t^{n}\right)
$$

em que U é a velocidade tridimensional. Separando a equação acima nas componentes vertical e horizontal como em [42], temos

$$
\begin{aligned}
& \frac{\sigma_{\mathbf{a}}^{n+1}-\sigma_{\mathbf{m}}^{n}}{\Delta t}=\dot{\sigma}^{n}\left(\mathbf{x}_{\mathbf{m}}, \sigma_{\mathbf{m}}\right) \\
& \frac{\mathbf{x}_{\mathbf{a}}^{n+1}-\mathbf{x}_{\mathbf{m}}^{n}}{\Delta t}=\mathbf{V}^{n}\left(\mathbf{x}_{\mathbf{m}}, \sigma_{\mathbf{m}}\right)
\end{aligned}
$$


Escrevemos agora as equações (1.20) e (1.21) como um esquema iterativo

$$
\begin{aligned}
\sigma_{\mathbf{m}}^{k+1} & =\sigma_{\mathbf{a}}-\tau \dot{\sigma}^{n}\left(\mathbf{x}_{\mathbf{a}}^{k}, \sigma_{\mathbf{a}}^{k}\right) \\
\mathbf{x}_{\mathbf{m}}^{k+1} & =b_{k}\left[\mathbf{x}_{\mathbf{a}}-\tau \mathbf{V}^{n}\left(\mathbf{x}_{\mathbf{m}}^{k}, \sigma_{\mathbf{m}}^{k+1}\right)\right]
\end{aligned}
$$

no qual

$$
\tau=\Delta t \quad \mathrm{e} \quad b_{k}=\frac{a}{\left.\| \mathbf{x}_{\mathbf{a}}-\tau \mathbf{V}^{n}\left(\mathbf{x}_{\mathbf{m}}^{k}, \sigma_{\mathbf{m}}^{k+1}\right)\right) \|}
$$

é um fator de correção para evitar que a partícula saia da esfera. Trabalhando com $b_{k}$, temos que

$$
\begin{aligned}
\left\langle\mathbf{x}_{\mathbf{a}}-\tau \mathbf{V}_{\mathbf{m}}^{n}, \mathbf{x}_{\mathbf{a}}-\tau \mathbf{V}_{\mathbf{m}}^{n}\right\rangle & =\left\|\mathbf{x}_{\mathbf{a}}\right\|^{2}-2 \tau\left\langle\mathbf{x}_{\mathbf{a}}, \mathbf{V}_{\mathbf{m}}^{n}\right\rangle+\tau^{2}\left\|\mathbf{V}_{\mathbf{m}}^{n}\right\|^{2} \\
& =a^{2}-2 \tau\left\langle\mathbf{x}_{\mathbf{a}}, \mathbf{V}_{\mathbf{m}}^{n}\right\rangle+\tau^{2}\left\|\mathbf{V}_{\mathbf{m}}^{n}\right\|^{2}
\end{aligned}
$$

desta forma, obtemos

$$
b_{k}=\left[1-\frac{2 \tau}{a^{2}}\left\langle\mathbf{x}_{\mathbf{a}}, \mathbf{V}_{\mathbf{m}}^{n}\right\rangle+\frac{\tau^{2}}{a^{2}}\left\|\mathbf{V}_{\mathbf{m}}^{n}\right\|^{2}\right]^{-\frac{1}{2}}
$$

Este esquema iterativo é resolvido da mesma forma que em [40]. Conhecendo $\mathbf{x}_{\mathbf{m}}^{k}$ correspondendo a $\left(\lambda_{\mathbf{m}}^{k}, \theta_{\mathbf{m}}^{k}, \sigma_{\mathbf{m}}^{k}\right)$, calculamos primeiro $\sigma_{\mathbf{m}}^{k+1}$ a partir de (1.22) e em seguida calculamos $\mathbf{V}^{n}\left(\lambda_{\mathbf{m}}^{k}, \theta_{\mathbf{m}}^{k}, \sigma_{\mathbf{m}}^{k+1}\right)=\left(u^{k}, v^{k}\right)$ para na sequência definir as velocidades normalizadas

$$
\tilde{u}=\frac{u^{k}}{a} \quad \text { e } \quad \tilde{v}=\frac{v^{k}}{a} .
$$

Se $(X, Y, Z)$ representam as coordenadas cartesianas normalizadas $\left(\frac{x}{a}, \frac{y}{a}, \frac{z}{a}\right)$, temos

$$
\begin{aligned}
\dot{X}_{\mathbf{m}}^{k} & =-\tilde{u}^{k} \sin \left(\lambda_{\mathbf{m}}^{k}\right)-\tilde{v} \cos \left(\lambda_{\mathbf{m}}^{k}\right) \sin \left(\theta_{\mathbf{m}}^{k}\right) \\
\dot{Y}_{\mathbf{m}}^{k} & =\tilde{u}^{k} \cos \left(\lambda_{\mathbf{m}}^{k}\right)-\tilde{v} \sin \left(\lambda_{\mathbf{m}}^{k}\right) \sin \left(\theta_{\mathbf{m}}^{k}\right) \\
\dot{Z}_{\mathbf{m}}^{k} & =\tilde{v} \cos \left(\theta_{\mathbf{m}}^{k}\right) .
\end{aligned}
$$

Agora, sejam $\lambda_{i}$ e $\theta_{j}$, respectivamente, as longitude e latitude do ponto de malha $\mathbf{x}_{\mathbf{a}}$. Logo, as suas correspondentes coordenadas cartesianas são

$$
\begin{aligned}
X_{\mathbf{a}} & =\cos \left(\lambda_{i}\right) \cos \left(\theta_{j}\right) \\
Y_{\mathbf{a}} & =\sin \left(\lambda_{i}\right) \cos \left(\theta_{j}\right) \\
Z_{\mathbf{a}} & =\sin \left(\theta_{j}\right) .
\end{aligned}
$$


Substituindo (1.26) e (1.27) em (1.24), obtemos o valor de $b_{k}$ :

$$
b_{k}=\left[1-2 \tau\left(X_{\mathbf{a}} \dot{X}_{\mathbf{m}}^{k}+Y_{\mathbf{a}} \dot{Y}_{\mathbf{m}}^{k}+Z_{\mathbf{a}} \dot{Z}_{\mathbf{m}}^{k}\right)+\tau^{2}\left(\left(\dot{X}_{\mathbf{m}}^{k}\right)^{2}+\left(\dot{Y}_{\mathbf{m}}^{k}\right)^{2}+\left(\dot{Z}_{\mathbf{m}}^{k}\right)^{2}\right)\right]^{-\frac{1}{2}}
$$

e a equação (1.23) pode ser reescrita da forma

$$
\begin{aligned}
X_{\mathbf{m}}^{k+1} & =b^{k}\left[X_{\mathbf{a}}-\tau \dot{X}_{\mathbf{m}}^{k}\right] \\
Y_{\mathbf{m}}^{k+1} & =b^{k}\left[Y_{\mathbf{a}}-\tau \dot{Y}_{\mathbf{m}}^{k}\right] \\
Z_{\mathbf{m}}^{k+1} & =b^{k}\left[Z_{\mathbf{a}}-\tau \dot{Z}_{\mathbf{m}}^{k}\right] .
\end{aligned}
$$

Completamos a iteração calculando

$$
\lambda_{\mathbf{m}}^{k+1}=\tan ^{-1}\left(\frac{Y_{\mathbf{m}}^{k+1}}{X_{\mathbf{m}}^{k+1}}\right) \quad \text { e } \quad \theta_{\mathbf{m}}^{k+1}=\sin ^{-1}\left(Z_{\mathbf{m}}^{k+1}\right) .
$$

No início da integração (primeiro passo no tempo) escolhemos $\mathbf{x}_{\mathbf{m}}^{0}=\mathbf{x}_{\mathbf{a}}$, que corresponde a um deslocamento nulo e fazemos várias iterações (4 ou 5) para calcular o ponto de partida, enquanto nos seguintes passos no tempo escolhemos o valor para $\mathbf{x}_{\mathbf{m}}^{0}$ como sendo o valor de $\mathbf{x}_{\mathbf{m}}$ na iteração prévia. Observamos que em geral a posição $\left(\lambda^{k}, \theta^{k}\right)$ encontra-se entre os pontos de malha $\left(\lambda_{i}, \theta_{j}\right)$, devido ao fato de utilizarmos interpolação linear para calcular $\tilde{u}^{k} \mathrm{e}$ $\tilde{v}^{k}$. Uma vez calculado o ponto médio da trajetória calculamos o ponto de partida por meio das fórmulas

$$
\begin{aligned}
\sigma_{\mathbf{d}} & =\sigma_{\mathbf{a}}-2 \tau \dot{\sigma}^{n}\left(\mathbf{x}_{\mathbf{m}}, \sigma_{\mathbf{m}}\right) \\
\mathbf{x}_{\mathbf{d}} & =\frac{2}{a^{2}}\left\langle\mathbf{x}_{\mathbf{m}}, \mathbf{x}_{\mathbf{a}}\right\rangle \mathbf{x}_{\mathbf{m}}-\mathbf{x}_{\mathbf{a}}
\end{aligned}
$$

Também precisamos determinar, como veremos posteriormente, o deslocamento angular $\alpha$ que o ponto médio faz com o ponto de chegada $\mathbf{x}_{\mathbf{a}}$ (ou com o ponto de partida $\mathbf{x}_{\mathbf{d}}$ ) e o ângulo $\gamma$ que o vetor unitário c, ao longo da trajetória considerada como um arco do grande círculo, faz com o vetor unitário na direção zonal $\mathbf{i}_{\mathbf{m}}$, determinado pelas coordenadas $\left(\lambda_{\mathbf{m}}, \theta_{\mathbf{m}}\right)$. Assim, temos que

$$
\left\langle\mathbf{x}_{\mathbf{a}}, \mathbf{x}_{\mathbf{m}}\right\rangle=\left\|\mathbf{x}_{\mathbf{a}}\right\||| \mathbf{x}_{\mathbf{m}} \| \cos (\alpha) .
$$

Logo,

$$
\alpha=\cos ^{-1}\left(X_{\mathbf{a}} X_{\mathbf{m}}+Y_{\mathbf{a}} Y_{\mathbf{m}}+Z_{\mathbf{a}} Z_{\mathbf{m}}\right) .
$$

Considere agora 


$$
\mathbf{c}=\left(X_{\mathbf{c}}, Y_{\mathbf{c}}, Z_{\mathbf{c}}\right)=\frac{\left(X_{\mathbf{a}}-X_{\mathbf{d}}, Y_{\mathbf{a}}-Y_{\mathbf{d}}, Z_{\mathbf{a}}-Z_{\mathbf{d}}\right)}{\left[\left(X_{\mathbf{a}}-X_{\mathbf{d}}\right)^{2}+\left(Y_{\mathbf{a}}-Y_{\mathbf{d}}\right)^{2}+\left(Z_{\mathbf{a}}-Z_{\mathbf{d}}\right)^{2}\right]^{\frac{1}{2}}}
$$

o vetor unitário ao longo da trajetória (no ponto médio da mesma) e $\mathbf{i}_{\mathbf{m}}$ o vetor unitário na direção zonal determinado pelo ponto $\left(\lambda_{\mathbf{m}}, \theta_{\mathbf{m}}\right)$. Temos

$$
\mathbf{i}_{\mathbf{m}}=\left(-\sin \left(\lambda_{\mathbf{m}}\right), \cos \left(\lambda_{\mathbf{m}}\right), 0\right),
$$

pois o ângulo $\gamma$ entre os vetores $\mathbf{c}$ e $\mathbf{i}_{\mathbf{m}}$ é dado por

$$
\gamma=\cos ^{-1}\left(-\sin \left(\lambda_{\mathbf{m}}\right) X_{\mathbf{c}}+\cos \left(\lambda_{\mathbf{m}}\right) Y_{\mathbf{c}}\right)
$$

\subsubsection{Discretização semi-Lagrangiana}

Vamos discretizar a equação do momento na forma vetorial, como é feito em [42], evitando desta forma o surgimento dos termos métricos e portanto um tratamento explícito deles. Estes termos métricos tomam valores cada vez maiores perto dos polos, pois são uma fonte de instabilidade nos modelos semi-lagrangianos. Dessa forma, discretização semi-Lagrangiana da equação do momento é

$$
\begin{aligned}
(\mathbf{V}+F \mathbf{k} \times \mathbf{V}+\tau \nabla G)_{H, \mathbf{a}}^{n+1}= & (\mathbf{V}-F \mathbf{k} \times \mathbf{V}-\tau \nabla G)_{H, \mathbf{d}}^{n-1} \\
& +\tau \mathcal{N}_{\mathbf{V}, \mathbf{a}}^{n}+\tau \mathcal{N}_{\mathbf{V}, \mathbf{d}}^{n},
\end{aligned}
$$

$\operatorname{com} F=\tau f$ e $\mathcal{N}_{\mathbf{V}}=-R T^{\prime} \nabla P$. Separando a equação acima por componentes, temos

$$
\begin{aligned}
\left(X_{u}\right)_{\mathbf{a}}^{n+1} \mathbf{i}_{\mathbf{a}}+\left(X_{v}\right)_{\mathbf{a}}^{n+1} \mathbf{j}_{\mathbf{a}}= & \left(Y_{u}\right)_{\mathbf{d}}^{n-1} \mathbf{i}_{\mathbf{d}}+\left(Y_{v}\right)_{\mathbf{d}}^{n-1} \mathbf{j}_{\mathbf{d}} \\
& +\tau\left(\mathcal{N}_{\mathbf{V}, \lambda}^{n}\right)_{\mathbf{a}} \mathbf{i}_{\mathbf{a}}+\tau\left(\mathcal{N}_{\mathbf{V}, \theta}^{n}\right)_{\mathbf{a}} \mathbf{j}_{\mathbf{a}} \\
& +\tau\left(\mathcal{N}_{\mathbf{V}, \lambda}^{n}\right)_{\mathbf{d}} \mathbf{i}_{\mathbf{d}}+\tau\left(\mathcal{N}_{\mathbf{V}, \theta}^{n}\right)_{\mathbf{d}} \mathbf{j}_{\mathbf{d}}
\end{aligned}
$$

em que 


$$
\begin{aligned}
X_{u} & =u-F v+\frac{\tau}{a \cos (\theta)} \frac{\partial G}{\partial \lambda} \\
X_{v} & =v+F u+\frac{\tau}{a} \frac{\partial G}{\partial \theta} \\
Y_{u} & =u+F v-\frac{\tau}{a \cos (\theta)} \frac{\partial G}{\partial \lambda} \\
Y_{v} & =v-F u-\frac{\tau}{a} \frac{\partial G}{\partial \theta} \\
\mathcal{N}_{\mathbf{V}, \lambda} & =-\frac{R T^{\prime}}{a \cos (\theta)} \frac{\partial P}{\partial \lambda} \\
\mathcal{N}_{\mathbf{V}, \theta} & =-\frac{R T^{\prime}}{a} \frac{\partial P}{\partial \theta}
\end{aligned}
$$

Da equação (1.37) observamos que temos uma igualdade de vetores horizontais sobre uma trajetória expressados em sistemas de coordenadas diferentes, estabelecidos nos pontos de chegada $\mathbf{x}_{\mathbf{a}}$ (com os vetores $\mathbf{i}_{\mathbf{a}}$ e $\mathbf{j}_{\mathbf{a}}$ formando uma base) e de partida $\mathbf{x}_{\mathbf{d}}$ (com os vetores $\mathbf{i}_{\mathbf{d}}$ e $\mathbf{j}_{\mathbf{d}}$ como base). Assim, é necessário expressar os termos de tal equação em um único sistema de coordenadas, estabelecido no ponto médio da trajetória $\mathbf{x}_{\mathbf{m}}$. Fazemos isto adotando a estratégia desenvolvida em [41], que consiste em projetar os vetores no plano tangente à esfera no ponto médio da trajetória e resolver as equações neste plano.

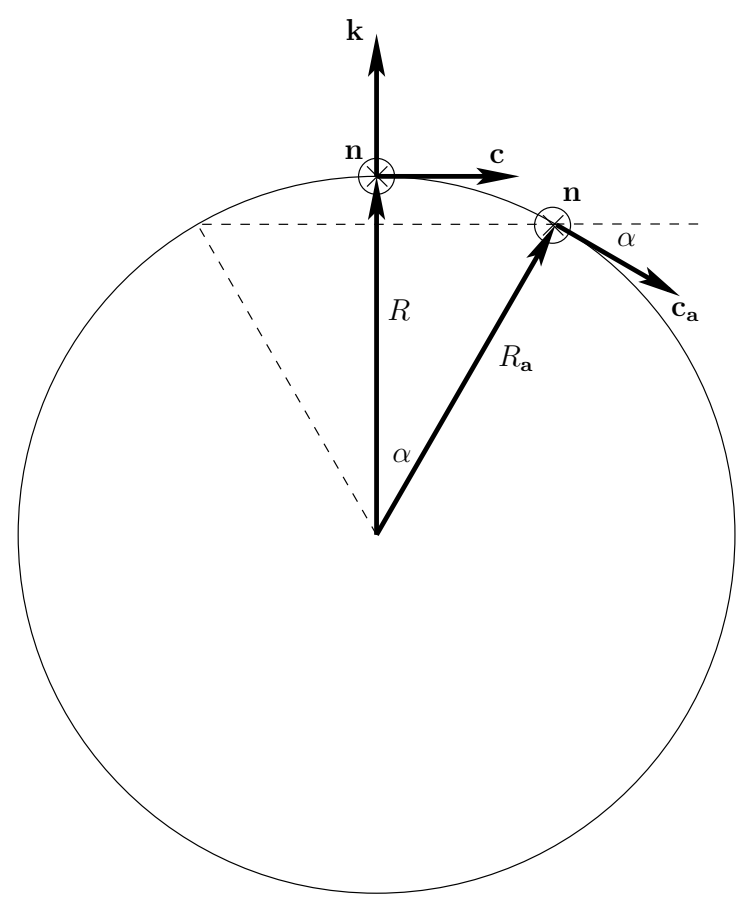

Figura 1.2: Vetores ao longo da trajetória. 
Vamos descrever agora este processo de forma resumida. Para encontrar a transformação de vetores horizontais em coordenadas esféricas sobre uma trajetória em um plano cartesiano tangente, definimos c como o vetor unitário na direção do vento no ponto médio da trajetória e $\mathbf{n}$ o vetor unitário a esquerda de $\mathbf{c}$, isto é devido $\mathbf{c}$ e $\mathbf{n}$ formarem uma outra base do plano gerado pelos vetores unitários $\mathbf{i}_{\mathbf{m}}$ e $\mathbf{j}_{\mathbf{m}}$. Como é mostrado na Figura 1.2, definimos $\alpha$ como o deslocamento angular positivo na direção de c, ao longo do grande círculo entre duas posições $R$ e $R_{\mathbf{a}}$, onde $R$ é o ponto médio da trajetória, e utilizamos os vetores $\mathbf{i}_{\mathbf{m}}$ e $\mathbf{j}_{\mathbf{m}}$ como base do plano cartesiano tangente a este ponto. Consideramos $R_{\mathbf{a}}$ sendo o ponto de chegada, pois os cálculos para o ponto de partida são análogos. Desta forma, para um dado vetor horizontal

$$
\mathbf{h}_{\mathbf{a}}=h_{\lambda} \mathbf{i}_{\mathbf{a}}+h_{\theta} \mathbf{j}_{\mathbf{a}}
$$

no ponto $R_{\mathbf{a}}$, fazer sua transformação ao plano cartesiano tangente em $R$, consiste em achar o vetor no plano gerado por $\left(\mathbf{i}_{\mathbf{m}}, \mathbf{j}_{\mathbf{m}}\right)$ com a mesma grandeza que $\mathbf{h}_{\mathbf{a}}$ e cuja direção seja a projeção de $\mathbf{h}_{\mathbf{a}}$ sobre este plano. Isto é feito expressando primeiro os vetores $\mathbf{i}_{\mathbf{a}}$ e $\mathbf{j}_{\mathbf{a}}$ em função dos vetores $\mathbf{c}_{\mathbf{a}}$ e $\mathbf{n}_{\mathbf{a}}$, onde $\mathbf{c}_{\mathbf{a}}$ é o vetor unitário na direção do vento no ponto $R_{\mathbf{a}} \mathrm{e}$ $\mathbf{n}_{\mathbf{a}}$ é o vetor unitário ortogonal a esquerda de $\mathbf{c}_{\mathbf{a}}$ (observe que $\mathbf{n}_{\mathbf{a}}=\mathbf{n}$ pois consideramos a trajetória circular). A seguir o vetor $\mathbf{c}_{\mathbf{a}}$ é rotado na direção de $\mathbf{c}$, gerando os vetores $\left(\mathbf{i}_{R}\right)_{\mathbf{a}} \mathrm{e}$ $\left(\mathbf{j}_{R}\right)_{\mathbf{a}}$, ambos no plano tangente gerado por $\mathbf{i}_{\mathbf{m}}$ e $\mathbf{j}_{\mathbf{m}}$, pois são uma combinação linear de $\mathbf{c}$ e n. Desta forma, o vetor

$$
h_{\lambda}\left(\mathbf{i}_{R}\right)_{\mathbf{a}}+h_{\theta}\left(\mathbf{j}_{R}\right)_{\mathbf{a}}
$$

tem o mesmo módulo que $\mathbf{h}_{\mathbf{a}}$ e a sua direção é a projeção de $\mathbf{h}_{\mathbf{a}}$ em tal plano. Finalmente $\mathbf{c}$ e $\mathbf{n}$ são expressados em termos de $\mathbf{i}_{\mathbf{m}}$ e $\mathbf{j}_{\mathbf{m}}$ para encontrar as coordenadas do vetor $\mathbf{h}_{\mathbf{a}}$ no plano tangente. Assim,

$$
\left(\mathbf{i}_{R}\right)_{\mathbf{a}}=\frac{Y_{\mathbf{a}} \mathbf{i}_{\mathbf{m}}+X_{\mathbf{a}} \mathbf{j}_{\mathbf{m}}}{\cos \left(\theta_{\mathbf{a}}\right)} \quad \text { e } \quad\left(\mathbf{j}_{R}\right)_{\mathbf{a}}=\frac{-X_{\mathbf{a}} \mathbf{i}_{\mathbf{m}}+Y_{\mathbf{a}} \mathbf{j}_{\mathbf{m}}}{\cos \left(\theta_{\mathbf{a}}\right)},
$$

em que

$$
\begin{aligned}
X_{\mathbf{a}} & =\sin (\alpha) \cos (\gamma) \sin \left(\theta_{\mathbf{a}}\right)+(1-\cos (\alpha)) \sin (\gamma) \cos (\gamma) \cos \left(\theta_{\mathbf{a}}\right) \\
Y_{\mathbf{a}} & =\cos \left(\theta_{\mathbf{a}}\right)-\sin (\alpha) \sin (\gamma) \sin \left(\theta_{\mathbf{a}}\right)-(1-\cos (\alpha)) \sin ^{2}(\gamma) \cos \left(\theta_{\mathbf{a}}\right)
\end{aligned}
$$

e $\gamma$ é o ângulo entre $\mathbf{c}$ e $\mathbf{i}_{\mathbf{m}}$. Aplicando esta transformação nos vetores $\mathbf{i}_{\mathbf{a}}, \mathbf{j}_{\mathbf{a}}$, $\mathbf{i}_{\mathbf{d}}$ e $\mathbf{j}_{\mathbf{d}}$ na equação (1.37), temos

$$
A\left(\frac{Y_{\mathbf{a}} \mathbf{i}_{\mathbf{m}}+X_{\mathbf{a}} \mathbf{j}_{\mathbf{m}}}{\cos \left(\theta_{\mathbf{a}}\right)}\right)+B\left(\frac{X_{\mathbf{a}} \mathbf{i}_{\mathbf{m}}+Y_{\mathbf{a}} \mathbf{j}_{\mathbf{m}}}{\cos \left(\theta_{\mathbf{a}}\right)}\right)=C\left(\frac{Y_{\mathbf{d}} \mathbf{i}_{\mathbf{m}}+X_{\mathbf{d}} \mathbf{j}_{\mathbf{m}}}{\cos \left(\theta_{\mathbf{d}}\right)}\right)+D\left(\frac{-X_{\mathbf{d}} \mathbf{i}_{\mathbf{m}}+Y_{\mathbf{d}} \mathbf{j}_{\mathbf{m}}}{\cos \left(\theta_{\mathbf{d}}\right)}\right),
$$

em que 


$$
\begin{aligned}
A & =\left(X_{u}\right)_{\mathbf{a}}^{n+1}-\tau\left(\mathcal{N}_{\mathbf{V}, \lambda}\right)_{\mathbf{a}}^{n} \\
B & =\left(X_{v}\right)_{\mathbf{a}}^{n+1}-\tau\left(\mathcal{N}_{\mathbf{V}, \theta}\right)_{\mathbf{a}}^{n} \\
C & =\left(Y_{u}\right)_{\mathbf{d}}^{n-1}+\tau\left(\mathcal{N}_{\mathbf{V}, \lambda}\right)_{\mathbf{d}}^{n} \\
D & =\left(Y_{v}\right)_{\mathbf{d}}^{n-1}+\tau\left(\mathcal{N}_{\mathbf{V}, \theta}\right)_{\mathbf{d}}^{n}
\end{aligned}
$$

Igualando por componentes a equação acima, obtemos o sistema

$$
\left\{\begin{array}{l}
\frac{Y_{\mathbf{a}}}{\cos \left(\theta_{\mathbf{a}}\right)} A-\frac{X_{\mathbf{a}}}{\cos \left(\theta_{\mathbf{a}}\right)} B=\frac{Y_{\mathbf{d}}}{\cos \left(\theta_{\mathbf{d}}\right)} C-\frac{X_{\mathbf{d}}}{\cos \left(\theta_{\mathbf{d}}\right)} D \\
\frac{X_{\mathbf{a}}}{\cos \left(\theta_{\mathbf{a}}\right)} A+\frac{Y_{\mathbf{a}}}{\cos \left(\theta_{\mathbf{a}}\right)} B=\frac{X_{\mathbf{d}}}{\cos \left(\theta_{\mathbf{d}}\right)} C+\frac{Y_{\mathbf{d}}}{\cos \left(\theta_{\mathbf{d}}\right)} D
\end{array}\right.
$$

que ao ser resolvido fica da forma

$$
\begin{aligned}
A & =\frac{X_{\mathbf{a}}}{\cos \left(\theta_{\mathbf{a}}\right)}\left(\frac{X_{\mathbf{d}} C+Y_{\mathbf{d}} D}{\cos \left(\theta_{\mathbf{d}}\right)}\right)+\frac{Y_{\mathbf{a}}}{\cos \left(\theta_{\mathbf{a}}\right)}\left(\frac{Y_{\mathbf{d}} C-X_{\mathbf{d}} D}{\cos \left(\theta_{\mathbf{d}}\right)}\right) \\
B & =\frac{-X_{\mathbf{a}}}{\cos \left(\theta_{\mathbf{a}}\right)}\left(\frac{Y_{\mathbf{d}} C-X_{\mathbf{d}} D}{\cos \left(\theta_{\mathbf{d}}\right)}\right)+\frac{Y_{\mathbf{a}}}{\cos \left(\theta_{\mathbf{a}}\right)}\left(\frac{X_{\mathbf{d}} C+Y_{\mathbf{d}} D}{\cos \left(\theta_{\mathbf{d}}\right)}\right),
\end{aligned}
$$

$\mathrm{ou}$

$$
\begin{aligned}
& \left(X_{u}\right)_{\mathbf{a}}^{n+1}=\frac{X_{\mathbf{a}}}{\cos \left(\theta_{\mathbf{a}}\right)}\left(\frac{X_{\mathbf{d}} C+Y_{\mathbf{d}} D}{\cos \left(\theta_{\mathbf{d}}\right)}\right)+\frac{Y_{\mathbf{a}}}{\cos \left(\theta_{\mathbf{a}}\right)}\left(\frac{Y_{\mathbf{d}} C-X_{\mathbf{d}} D}{\cos \left(\theta_{\mathbf{d}}\right)}\right)+\tau\left(\mathcal{N}_{\mathbf{V}, \lambda}\right)_{\mathbf{a}}^{n} \equiv R_{u} \\
& \left(X_{v}\right)_{\mathbf{a}}^{n+1}=\frac{-X_{\mathbf{a}}}{\cos \left(\theta_{\mathbf{a}}\right)}\left(\frac{Y_{\mathbf{d}} C-X_{\mathbf{d}} D}{\cos \left(\theta_{\mathbf{d}}\right)}\right)+\frac{Y_{\mathbf{a}}}{\cos \left(\theta_{\mathbf{a}}\right)}\left(\frac{X_{\mathbf{d}} C+Y_{\mathbf{d}} D}{\cos \left(\theta_{\mathbf{d}}\right)}\right)+\tau\left(\mathcal{N}_{\mathbf{V}, \theta}\right)_{\mathbf{a}}^{n} \equiv R_{v} .
\end{aligned}
$$

Definindo a matriz $\mathbf{C}$ por

$$
\mathbf{C}=\left(\begin{array}{cc}
1 & -F \\
F & 1
\end{array}\right)
$$

podemos escrever novamente as duas equações anteriores na forma vetorial, como

$$
(\mathbf{C V}+\tau \nabla G)_{\mathbf{a}}^{n+1}=R_{\mathbf{V}}
$$

$\operatorname{com} R_{\mathbf{V}}=\left(R_{u}, R_{v}\right)^{T}$, ou ainda mais

$$
\mathbf{V}_{\mathbf{a}}^{n+1}+\tau\left(\mathbf{C}^{-1} \nabla G\right)_{\mathbf{a}}^{n+1}=\mathbf{C}_{\mathbf{a}}^{-1} R_{\mathbf{V}}
$$

Ao aplicarmos o divergente na equação (1.38), obtemos 


$$
\nabla \cdot \mathbf{V}_{\mathbf{a}}^{n+1}+\tau \nabla \cdot\left(\mathbf{C}^{-1} \nabla G\right)_{\mathbf{a}}^{n+1}=\nabla \cdot\left(\mathbf{C}_{\mathbf{a}}^{-1} R_{\mathbf{V}}\right)
$$

Agora discretizamos a equação da continuidade (1.12) obtendo

$$
\begin{aligned}
\left(l^{\prime}+\tau \int_{0}^{1} \nabla \cdot \mathbf{V} d \sigma\right)_{\mathbf{a}, 2}^{n+1}= & \left(l^{\prime}-\tau \int_{0}^{1} \nabla \cdot \mathbf{V} d \sigma\right)_{\mathbf{d}, 2}^{n-1} \\
& +\tau \mathcal{N}_{l^{\prime}, \mathbf{a}}^{n}+\tau \mathcal{N}_{l^{\prime}, \mathbf{d}}^{n}=R_{l^{\prime}}
\end{aligned}
$$

$\operatorname{com} \mathcal{N}_{l^{\prime}}=\frac{1}{R T_{0}}\left(\overline{\mathbf{V}} \cdot \nabla \Phi_{s}\right)$. Finalmente, a discretização da equação da termodinâmica (1.14) resulta em

$$
\left(T-\tau \mathcal{L}_{T}\right)_{\mathbf{a}}^{n+1}=\left(T+\tau \mathcal{L}_{T}\right)_{\mathbf{d}}^{n-1}+\tau \mathcal{N}_{T, \mathbf{a}}^{n}+\tau \mathcal{N}_{T, \mathbf{d}}^{n}=R_{T}
$$

em que

$$
\mathcal{L}_{T}=\frac{\kappa T_{0}}{\sigma}\left(-(1-\sigma) \int_{0}^{1} \nabla \cdot \mathbf{V} d \sigma+\int_{\sigma}^{1} \nabla \cdot \mathbf{V} d \sigma\right)-\kappa T_{0} \int_{0}^{1} \nabla \cdot \mathbf{V} d \sigma
$$

$\mathrm{e}$

$$
\begin{aligned}
\mathcal{N}_{T}= & \kappa T^{\prime}\left(\frac{\dot{\sigma}}{\sigma}-\int_{0}^{1} \nabla \cdot \mathbf{V} d \sigma\right)+\kappa T\left(-\int_{0}^{1} \mathbf{V} \cdot \nabla P d \sigma+\mathbf{V} \cdot \nabla P\right) \\
& +\frac{\kappa T_{0}}{\sigma}\left(-(1-\sigma) \int_{0}^{1} \mathbf{V} \cdot \nabla P d \sigma+\int_{\sigma}^{1} \mathbf{V} \cdot \nabla P d \sigma\right) .
\end{aligned}
$$

\subsection{Discretização vertical}

Dividimos a atmosfera em $N_{\sigma}$ camadas verticais numeradas de 1 a $N_{\sigma}$, desde a superfície terrestre até o topo da atmosfera, obtendo na $k$-ésima camada uma largura $\Delta \sigma_{k}$. Representamos cada variável do modelo no ponto do meio de cada camada, com exceção da velocidade vertical $\dot{\sigma}$, que é definida nos extremos das camadas, tal como podemos observar na Figura 1.3. Para aproximar as integrais verticais utilizamos a regra do ponto médio com repetição. Nesta seção, consideramos as variáveis do modelo como vetores de campos bidimensionais, sendo cada componente desse vetor o valor da variável na correspondente camada vertical. 


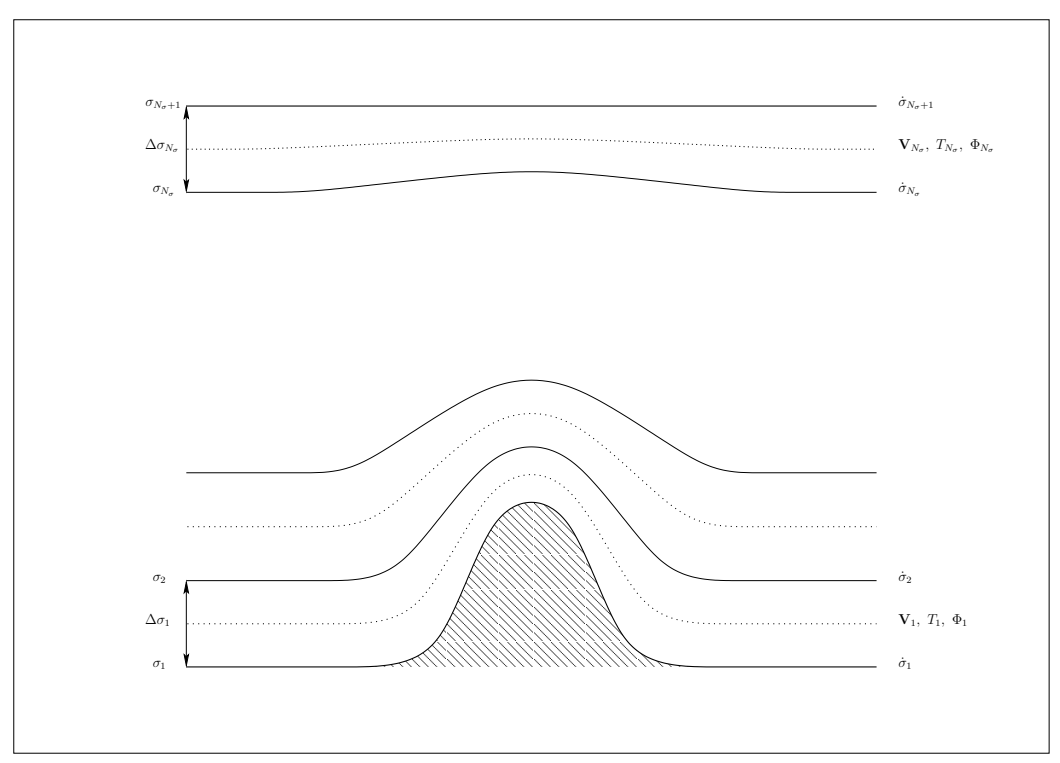

Figura 1.3: Divisão da atmosfera em camadas e posição das variáveis nas mesmas.

\section{Discretização vertical da equação do momento}

Expressando a equação (1.39) em cada camada e mantendo contínua a variação na horizontal, temos

$$
D_{\mathbf{a}, k}^{n+1}=-\tau \nabla \cdot\left(\mathbf{C}^{-1} \nabla G_{k}\right)_{\mathbf{a}}^{n+1}+\nabla \cdot\left(\mathbf{C}_{\mathbf{a}}^{-1} R_{\mathbf{V}, k}\right), \quad k=1, \ldots, N_{\sigma}
$$

e definindo os vetores $N_{\sigma}$-dimensionais $\mathbf{D}, \mathbf{G}$ e $\mathbf{R}_{\mathbf{V}}$ cujos elementos são os termos $D_{k}, G_{k}$ e $R_{\mathbf{V}, k}$, respectivamente, a equação anterior fica da seguinte forma

$$
\mathbf{D}_{\mathbf{a}}^{n+1}=-\tau \nabla \cdot\left(\mathbf{C}^{-1} \nabla \mathbf{G}\right)_{\mathbf{a}}^{n+1}+\nabla \cdot\left(\mathbf{C}_{\mathbf{a}}^{-1} \mathbf{R}_{\mathbf{V}}\right)
$$

\section{Discretização vertical da equação hidrostática}

Discretizando a equação (1.7), obtemos

$$
\sum_{k=1}^{N_{\sigma}} \Phi_{k} \Delta \sigma_{k}=\Phi_{s}+R \sum_{k=1}^{N_{\sigma}} T_{k} \sigma_{k}
$$

e ao discretizarmos a equação (1.8) nos extremos de cada camada, temos que

$$
\left(\Phi_{k-1}-\Phi_{k}\right)+C_{p}\left(\frac{\Theta_{k}+\Theta_{k-1}}{2}\right)\left(\Pi_{k-1}-\Pi_{k}\right)=0, \quad k=1, \ldots, N_{\sigma} .
$$

Logo, 


$$
\begin{aligned}
\Phi_{k-1}-\Phi_{k} & =\frac{C_{p}}{2}\left(\Theta_{k}+\Theta_{k-1}\right)\left(\Pi_{k}-\Pi_{k-1}\right) \\
& =C_{p}\left[\frac{\Theta_{k}}{2}\left(\Pi_{k}-\Pi_{k-1}\right)+\frac{\Theta_{k-1}}{2}\left(\Pi_{k}-\Pi_{k-1}\right)\right] \\
& =C_{p}\left[\frac{\Theta_{k} \Pi_{k}}{2}\left(1-\frac{\Pi_{k-1}}{\Pi_{k}}\right)+\frac{\Theta_{k-1} \Pi_{k-1}}{2}\left(\frac{\Pi_{k}}{\Pi_{k-1}}-1\right)\right] \\
& =C_{p}\left[T_{k-1} h_{1}(k-1)+T_{k} h_{2}(k-1)\right],
\end{aligned}
$$

em que

$$
h_{1}(k-1)=\frac{1}{2}\left(\frac{\Pi_{k}}{\Pi_{k-1}}-1\right), \quad h_{2}(k-1)=\frac{1}{2}\left(1-\frac{\Pi_{k-1}}{\Pi_{k}}\right) .
$$

Definimos os vetores $N_{\sigma}$-dimensionais $\boldsymbol{\Phi}$ e $\mathbf{T}$ de forma análoga ao vetor $\mathbf{D}$ e ao juntarmos as equações (1.45) e (1.46), obtemos

$$
\mathbf{A}_{\mathbf{1}} \mathbf{\Phi}=C_{p} \mathbf{H} \mathbf{T}+\widetilde{\boldsymbol{\Phi}}_{\boldsymbol{s}}
$$

onde $\widetilde{\boldsymbol{\Phi}}_{\boldsymbol{s}}=\left(0, \ldots, 0, \Phi_{s}\right)^{t}$

$$
\mathbf{A}_{\mathbf{1}}=\left(\begin{array}{cccccc}
1 & -1 & & & & \\
& & & & \\
& & & & \\
& & \ddots & \ddots & & \\
& & & & & \\
& & & & 1 & -1 \\
\Delta \sigma_{1} & \ldots & & & & \Delta \sigma_{N_{\sigma}}
\end{array}\right)
$$

e

$$
\mathbf{H}=\left(\begin{array}{cccccc}
h_{1}(1) & h_{2}(1) & & & & \\
& & & & \\
& & & & & \\
& & \ddots & \ddots & & \\
& & & & & \\
& & & & h_{1}\left(N_{\sigma}-1\right) & h_{2}\left(N_{\sigma}-1\right) \\
\kappa \Delta \sigma_{1} & \ldots & & & & \kappa \Delta \sigma_{N_{\sigma}}
\end{array}\right)
$$

$\mathrm{Ou}$

$$
\Phi=\mathbf{A T}+\boldsymbol{\Phi}_{s}
$$

$\operatorname{com} \mathbf{A}=C_{p} \mathbf{A}_{\mathbf{1}}{ }^{-1} \mathbf{H}, \quad \Phi_{s}=\mathbf{A}_{\mathbf{1}}{ }^{-1} \widetilde{\boldsymbol{\Phi}}_{\boldsymbol{s}}=\left(\Phi_{s}, \ldots, \Phi_{s}\right)^{t} \mathrm{e}$ 


$$
\mathbf{A}_{1}^{-1}=\left(\begin{array}{ccccccc}
\sigma_{2} & \sigma_{3} & \cdots & \sigma_{k} & \cdots & \sigma_{N_{\sigma}} & 1 \\
\sigma_{2}-1 & & & & & & 1 \\
\vdots & & & & & & \vdots \\
& & & \sigma_{k} & & & \\
& & & \sigma_{k}-1 & \ddots & & \\
& & & & \ddots & N_{\sigma} & 1 \\
\sigma_{2}-1 & \ldots & & \sigma_{k}-1 & & \sigma_{N_{\sigma}}-1 & 1
\end{array}\right)
$$

\section{Discretização vertical da equação da continuidade}

Discretizando a equação (1.40) obtemos

$$
l_{\mathbf{a}, 2}^{\prime n+1}+\tau \sum_{k=1}^{N_{\sigma}} D_{k}^{n+1} \Delta \sigma_{k}=R_{l^{\prime}}
$$

Com o objetivo de termos uma equação matricial, repetimos a equação anterior $N_{\sigma}$ vezes e definimos os vetores $N_{\sigma^{-}}$dimensionais $\mathbf{I}^{\prime}$ e $\mathbf{R}_{\mathbf{l}^{\prime}}$, de forma análoga ao vetor $\mathbf{D}$. Definimos também a matriz diagonal $\boldsymbol{\Delta}$ tal que $\boldsymbol{\Delta}_{k, k}=\Delta \sigma_{k}$ e a matriz $\mathbf{1}$, cujos elementos são iguais a 1. Assim, a equação anterior será dada por

$$
\mathbf{l}_{\mathbf{a}, 2}^{n+1}+\tau \mathbf{1} \Delta \mathbf{D}_{\mathbf{a}, 2}^{n+1}=\mathbf{R}_{\mathbf{l}^{\prime}}
$$

\section{Discretização vertical da equação termodinâmica}

Discretizando a equação (1.41), no ponto do meio de cada camada, temos

$$
T_{\mathbf{a}, k}^{n+1}-\tau \mathcal{L}_{T}\left(T_{\mathbf{a}, k}^{n+1}\right)=R_{T, k}, \quad k=1, \ldots, N_{\sigma}
$$

$\operatorname{com} \mathcal{L}_{T}$ definido como anteriormente. Da definição da pressão normalizada, observamos que

$$
\Pi=\left(\frac{p}{p_{0}}\right)^{\kappa}=\left(\frac{p / p_{s}}{p_{0} / p_{s}}\right)^{\kappa}=\left(\frac{\sigma}{\sigma_{0}}\right)^{\kappa},
$$

dado que

$$
\frac{\partial \Pi}{\partial \sigma}=\kappa\left(\frac{\sigma}{\sigma_{0}}\right)^{\kappa-1} \frac{1}{\sigma_{0}},
$$

com o qual

$$
\frac{1}{\Pi} \frac{\partial \Pi}{\partial \sigma}=\left(\frac{\sigma_{0}}{\sigma}\right)^{\kappa}\left(\frac{\sigma}{\sigma_{0}}\right)^{\kappa-1} \frac{\kappa}{\sigma_{0}}=\frac{\kappa}{\sigma} .
$$

Desta forma, o termo linear $\mathcal{L}_{T}$ da equação da termodinâmica, para uma camada arbitrária, fica como 


$$
\begin{aligned}
\mathcal{L}_{T}\left(T_{k}\right)= & -\kappa T_{0} \int_{0}^{1} \nabla \cdot \mathbf{V} d \sigma+\frac{T_{0}}{\Pi_{k} \Delta \sigma_{k}}(\Delta \Pi)_{k} \times \\
& \left(-\left(1-\sigma_{k+\frac{1}{2}}\right) \int_{0}^{1} \nabla \cdot \mathbf{V} d \sigma+\int_{\sigma_{k+\frac{1}{2}}}^{1} \nabla \cdot \mathbf{V} d \sigma\right), \quad k=1, \ldots, N_{\sigma} .
\end{aligned}
$$

Aproximamos o segundo termo do lado direito da equação acima pela média de seus valores nos extremos da camada, assim, a equação anterior será dada por (veja [16] para maiores detalhes)

$$
\begin{aligned}
\mathcal{L}_{T}\left(T_{k}\right)= & -\kappa T_{0} \sum_{j=1}^{N_{\sigma}} D_{j} \Delta \sigma_{j}+ \\
& \frac{T_{0}}{\Delta \sigma_{k}}\left[\left(\left(1-\sigma_{k}\right) h_{2}(k-1)+\left(1-\sigma_{k+1}\right) h_{1}(k)\right) \sum_{j=1}^{N_{\sigma}} D_{j} \Delta \sigma_{j}-\right. \\
& \left.\left(h_{2}(k-1) \sum_{j=1}^{k-1} D_{j}+h_{1}(k) \sum_{j=1}^{k} D_{j} \Delta \sigma_{j}\right)\right] .
\end{aligned}
$$

Observamos que

$$
\left(\mathbf{H}_{\mathbf{1}} \mathbf{S}_{\mathbf{1}} \mathbf{1} \boldsymbol{\Delta} \mathbf{D}\right)_{k}=\left(\left(1-\sigma_{k}\right) h_{2}(k-1)+\left(1-\sigma_{k+1}\right) h_{1}(k)\right) \sum_{j=1}^{N_{\sigma}} D_{j} \Delta \sigma_{j}, \quad k=1, \ldots, N_{\sigma}
$$

e que

$$
\left(\mathbf{H}_{\mathbf{1}} \mathbf{1}_{\mathbf{L}} \mathbf{\Delta} \mathbf{D}\right)_{k}=h_{2}(k-1) \sum_{j=1}^{k-1} D_{j}+h_{1}(k) \sum_{j=1}^{k} D_{j} \Delta \sigma_{j}, \quad k=1, \ldots, N_{\sigma}
$$

em que

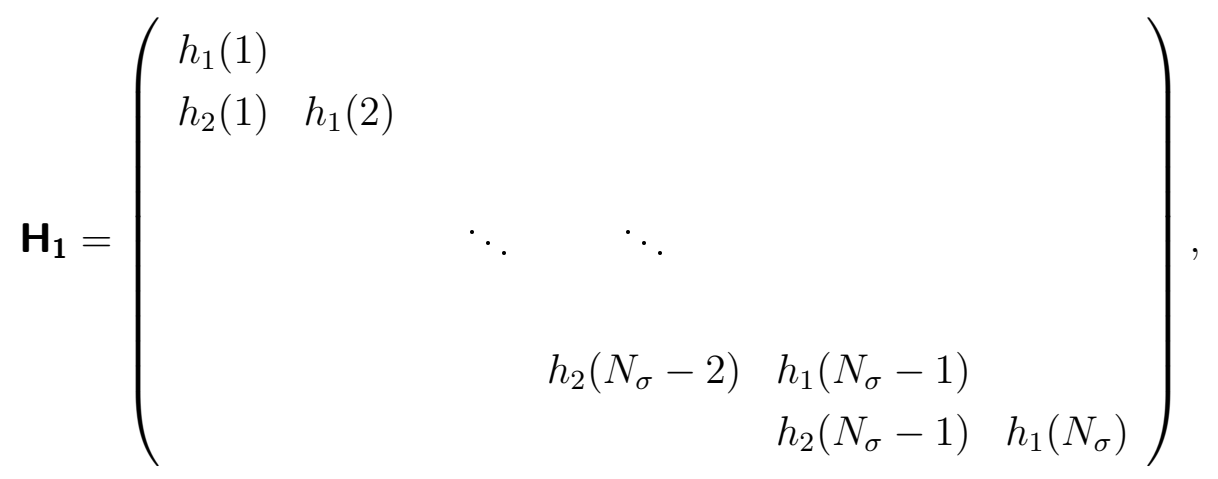




$$
\mathbf{S}_{\mathbf{1}}=\left(\begin{array}{ccc}
1-\sigma_{2} & & \\
& \ddots & \\
& & \\
& & 1-\sigma_{N_{\sigma}+1}
\end{array}\right),
$$

e $\mathbf{1}_{\mathbf{L}}$ é a matriz triangular inferior com elementos iguais a 1, pois temos que

$$
\begin{aligned}
\mathcal{L}_{T}(\mathbf{T}) & =T_{0}\left[-\kappa \mathbf{1} \boldsymbol{\Delta} \mathbf{D}+\boldsymbol{\Delta}^{-1}\left(\mathbf{H}_{\mathbf{1}} \mathbf{S}_{\mathbf{1}} \mathbf{1} \boldsymbol{\Delta} \mathbf{D}-\mathbf{H}_{\mathbf{1}} \mathbf{1}_{\mathbf{L}} \boldsymbol{\Delta} \mathbf{D}\right)\right] \\
& =T_{0} \boldsymbol{\Delta}^{-1}\left[-\kappa \boldsymbol{\Delta} \mathbf{1}+\mathbf{H}_{\mathbf{1}}\left(\mathbf{S}_{\mathbf{1}} \mathbf{1}-\mathbf{1}_{\mathbf{L}}\right)\right] \boldsymbol{\Delta} \mathbf{D}
\end{aligned}
$$

Definindo as matrizes

$$
\mathbf{A}_{\mathbf{2}}=\left(\begin{array}{c|c} 
& \kappa \Delta \sigma_{1} \\
0_{N_{\sigma} \times\left(N_{\sigma}-1\right)} & \vdots \\
\\
\\
& \kappa \Delta \sigma_{N_{\sigma}}
\end{array}\right)
$$

$\mathrm{e}$

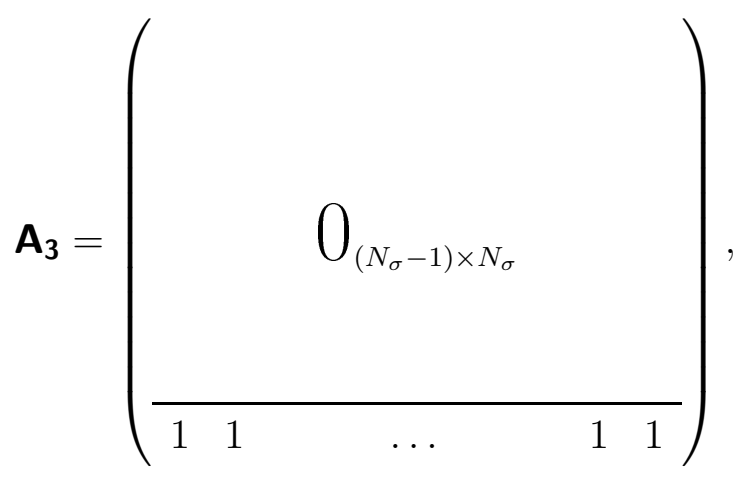

observamos que
a) $\kappa \Delta \mathbf{1}=\mathbf{A}_{\mathbf{2}} \mathbf{A}_{\mathbf{3}}$
b) $\mathbf{H}_{\mathbf{1}}=\mathbf{H}^{t}-\mathbf{A}_{\mathbf{2}}$
c) $\mathbf{S}_{\mathbf{1}} \mathbf{1}-\mathbf{1}_{\mathbf{L}}=\mathbf{A}_{\mathbf{3}}-\left(\mathbf{A}_{\mathbf{1}}^{-1}\right)^{t}$.

Substituindo os valores acima na equação (1.50) temos 


$$
\mathcal{L}_{T}(\mathbf{T})=-T_{0} \boldsymbol{\Delta}^{-1} \mathbf{H}^{t}\left(\mathbf{A}_{\mathbf{1}}{ }^{-1}\right)^{t} \boldsymbol{\Delta} \mathbf{D},
$$

do qual finalmente obtemos a discretização vertical da equação da termodinâmica

$$
\mathbf{T}_{\mathbf{a}}^{n+1}+\tau \mathbf{B D}_{\mathbf{a}}^{n+1}=\mathbf{R}_{\boldsymbol{T}}
$$

em que $\mathbf{B}=T_{0} \boldsymbol{\Delta}^{-1} \mathbf{H}^{t}\left(\mathbf{A}_{\mathbf{1}}^{-1}\right)^{t} \boldsymbol{\Delta}$ é a matriz que acopla as equações nas camadas verticais. Assim, o sistema a ser resolvido é composto pelas equações

$$
\begin{aligned}
& \mathbf{D}_{\mathbf{a}}^{n+1}+\tau \nabla \cdot\left(\mathbf{C}^{-1} \nabla \mathbf{G}\right)_{\mathbf{a}}^{n+1}=\nabla \cdot\left(\mathbf{C}_{\mathbf{a}}^{-1} \mathbf{R}_{\mathbf{V}}\right) \\
& \mathbf{I}_{\mathbf{a}, 2}^{\prime n+1}+\tau \mathbf{1} \Delta \mathbf{D}_{\mathbf{a}, 2}^{n+1}=\mathbf{R}_{\boldsymbol{l}^{\prime}} \\
& \mathbf{T}_{\mathbf{a}}^{n+1}+\tau \mathbf{B D}_{\mathbf{a}}^{n+1}=\mathbf{R}_{\boldsymbol{T}} .
\end{aligned}
$$

Combinando as equações (1.11), (1.47) e (1.53) obtemos

$$
\mathbf{G}_{\mathbf{a}}^{n+1}-\mathbf{A} \mathbf{T}_{\mathbf{a}}^{n+1}+\tau R T_{0} \mathbf{1} \Delta \mathbf{D}_{\mathbf{a}}^{n+1}=R T_{0} \mathbf{R}_{\boldsymbol{l}^{\prime}}
$$

Utilizamos a equação (1.54) para substituir o termo $\mathbf{A T}_{\mathbf{a}}^{n+1}$ na equação acima, obtendo

$$
\mathbf{G}_{\mathbf{a}}^{n+1}+\tau \mathbf{E D}_{\mathbf{a}}^{n+1}=R T_{0} \mathbf{R}_{\boldsymbol{l}^{\prime}}+\mathbf{A R}_{\boldsymbol{T}}
$$

com $\mathbf{E}=\mathbf{A B}+R T_{0} \mathbf{1} \boldsymbol{\Delta}$. Utilizamos agora a equação (1.52) para substituir o divergente na equação anterior, obtendo a seguinte equação tridimensional (elíptica) escalar para o geopotencial generalizado

$$
\mathbf{G}_{\mathbf{a}}^{n+1}-\tau^{2} \mathbf{E} \nabla \cdot\left(\mathbf{C}^{-1} \nabla \mathbf{G}\right)_{\mathbf{a}}^{n+1}=\mathbf{R}_{\boldsymbol{G}},
$$

onde $\mathbf{R}_{\boldsymbol{G}}=-\tau \mathbf{E} \nabla \cdot\left(\mathbf{C}_{\mathbf{a}}^{-1} \mathbf{R}_{\mathbf{V}}\right)+R T_{0} \mathbf{R}_{\mathbf{l}^{\prime}}+\mathbf{A R}_{\boldsymbol{T}}$. Observamos que a matriz $\mathbf{E}$ determina o caráter desta equação.

Em [16] foi mostrado que a matriz $\mathbf{E}$ é simétrica e positiva definida, dada por um sistema completo de autovetores e autovalores reais positivos. Seja $\boldsymbol{\Lambda}$ a matriz diagonal contendo os autovalores de $\mathbf{E}$ e $\mathbf{L}$ a matriz ortogonal que diagonaliza $\mathbf{E}$. Ao multiplicarmos a equação (1.55) por $\mathbf{L}^{-1}$, obtemos

$$
\mathbf{L}^{-1} \mathbf{G}_{\mathbf{a}}^{n+1}-\tau^{2} \mathbf{\Lambda} \mathbf{L}^{-1} \nabla \cdot\left(\mathbf{C}^{-1} \nabla \mathbf{G}\right)_{\mathbf{a}}^{n+1}=\mathbf{L}^{-1} \mathbf{R}_{\boldsymbol{G}}
$$

$\mathrm{Ou}$

$$
\widehat{\mathbf{G}}_{\mathbf{a}}^{n+1}-\tau^{2} \boldsymbol{\Lambda} \nabla \cdot\left(\mathbf{C}^{-1} \nabla \widehat{\mathbf{G}}\right)_{\mathbf{a}}^{n+1}=\mathbf{R}_{\widehat{\mathbf{G}}},
$$

onde $\widehat{\mathbf{G}}=\mathbf{L}^{-1} \mathbf{G}$ e $\mathbf{R}_{\widehat{\mathbf{G}}}=\mathbf{L}^{-1} \mathbf{R}_{\boldsymbol{G}}$. 
Observamos de (1.56) que a multiplicação da equação (1.55) pela matriz $\mathbf{L}^{-1}$ desacopla essa equação tridimensional em um sistema de $N_{\sigma}$ equações bidimensionais do tipo Helmholtz, as quais são elípticas uma vez que os autovalores de $\mathbf{E}$ são positivos. Uma vez resolvido o sistema de equações (1.56), calculamos o potencial generalizado $\mathbf{G}$ fazendo $\mathbf{G}=\mathbf{L} \widehat{\mathbf{G}}$ e utilizamo-lo para calcular o vento horizontal e a divergência a partir das equações (1.38) e (1.52), respectivamente. Finalmente utilizamos a divergência para calcular a temperatura e o logaritmo de pressão, respectivamente, a partir das equações (1.53) e (1.54).

\subsection{Discretização horizontal}

Utilizamos uma discretização em diferenças finitas de segunda ordem sobre uma malha uniforme $(\lambda, \theta)$ longitude/latitude do tipo C de Arakawa [8] (veja a Figura 1.4). Discretizamos cada uma das $N_{\sigma}$ equações de tipo Helmholtz de (1.56) em coordenadas esféricas. Trabalhamos a singularidade no polo por meio de uma forma integral e o uso de quadraturas, como dado em [15].

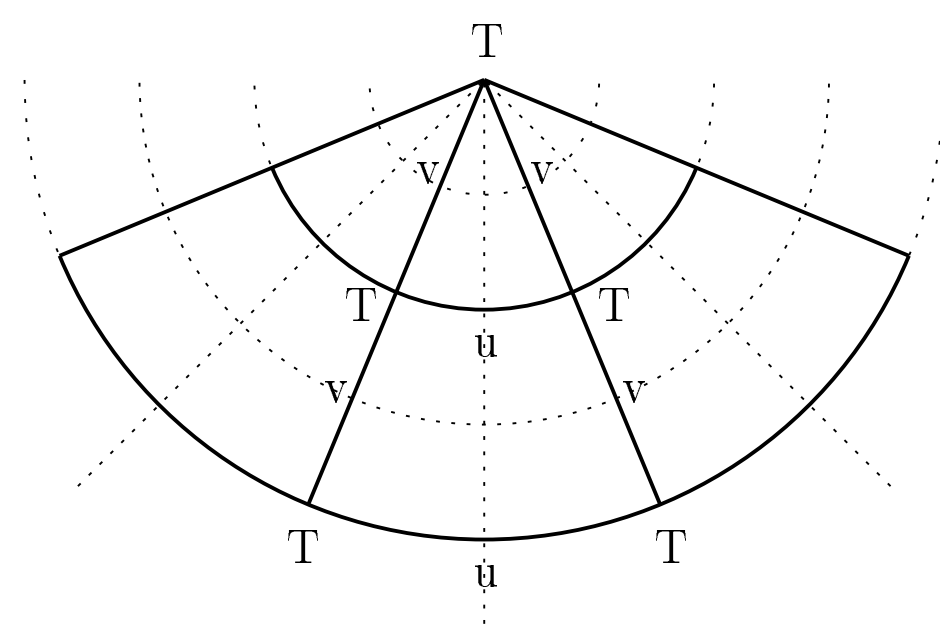

Figura 1.4: Malha $C$ e variáveis do modelo perto do polo.

\subsection{Solução numérica}

\subsubsection{Método Multigrid}

Resolvemos as equações (1.56) através de um método multigrid similar ao de [13]. Para cada ciclo utilizamos um esquema FAS (Full Approximation Scheme), o qual é compatível com a utilização de malhas com refinamento local. Como método de suavização empregamos um por linhas do tipo Zebra e o ciclo é o do tipo $\mathrm{V}(1,1)$. Para obter uma boa aproximação 
inicial utilizamos o modo FMG. Para obter maior informação sobre o método multigrid e seus componentes, veja [21], [52], [27] e [50].

\subsubsection{Resumo de um passo no tempo}

Conhecidos os campos do modelo na malha computacional para os tempos $t^{n-1}$ e $t^{n}$, precisamos efetuar os seguintes passos para determinar os valores dos campos para o tempo $t^{n+1}$ :

1. Calcular a velocidade vertical $\dot{\sigma}$ em cada ponto de malha de acordo com a forma discreta da equação (1.13).

2. Determinar os pontos de partida para cada ponto de malha através do processo iterativo (1.28)-(1.30). Os detalhes sobre a geometria esférica são tratados como em [40]. Observe que devido ao uso da malha C é necessário o cálculo do ponto de partida para os pontos $G, u$ e $v$. Porém, somente é calculado o ponto de partida correspondente ao ponto $G$. Os outros dois pontos de partida, correspondentes aos pontos $u$ e $v$ são calculados por interpolação linear.

3. Calcular a velocidade horizontal média (na vertical) e os correspondentes pontos de partida bidimensionais através de um processo iterativo análogo a (1.23), mas com o vento independente de $\sigma$.

4. Calcular os lados direito das equações (1.38), (1.40) e (1.41). Observamos que é necessário interpolar os termos nos pontos de partida.

5. Transformar os lados direito das equações do momento para ter as mesmas na forma por componentes (para mais detalhes ver [41]).

6. Calcular o lado direito da equação (1.55) seguida da multiplicação pela (pré-calculada) inversa da matriz $\mathbf{L}$, formada pelos autovalores de $\mathbf{E}$.

7. Resolver pelo método multigrid as $N_{\sigma}$ equações bidimensionais que resultam da equação (1.56), associadas aos autovalores da matriz $\mathbf{E}$.

8. Multiplicar as soluções de (1.56) por $\mathbf{L}$ para obter o geopotencial generalizado $G$.

9. Calcular o vento horizontal a partir da equação (1.38).

10. Calcular o divergente, $l^{\prime}$ e a temperatura $T$ a partir de (1.52)-(1.54).

Observamos que a matriz $\mathbf{E}$ independe do tempo e espaço, e a calculamos com antecedência, assim como os seus autovalores e autovetores (matrizes $\boldsymbol{\Lambda}$ e $\mathbf{L}$ ). 


\subsubsection{Malhas com refinamento local}

Perante a impossibilidade (memória e tempo de execução) de executar um modelo global com malha uniforme em alta resolução, surge a necessidade de encontrar alternativas para contornar este problema. Uma delas é a utilização de malhas com refinamento local, que nos possibilita a obtenção de resultados em alta resolução em uma região específica (que chamamos de região de interesse) sem utilizar a mesma resolução no resto do globo. Esta técnica tem como vantagens a redução do custo computacional em relação a um modelo global com malha uniforme em alta resolução e evita o uso de tratamentos especiais junto a fronteira, como no caso de modelos regionais, uma vez que mantemos um modelo global.

Desta forma, utilizamos uma malha uniforme e relativamente grossa sobre toda a esfera, a qual chamamos de malha base e a utilizamos na integração global. A seguir, colocamos malhas retangulares cada vez mais finas (razão 2 entre os espaçamentos de duas malhas consecutivas) entre duas longitudes e duas latitudes dadas, sem encostar nestas últimas, englobando uma certa região de interesse (não consideramos os polos dentro da área de interesse). Assim, ficam determinadas uma malha base, uma malha fina e uma zona de transição, devendo ter as malhas desta última pelo menos três linhas/colunas. A função da zona de transição é a de permitir uma transição suave entre as malhas base e fina, como podemos observar na Figura 1.5. A forma em que definimos os refinamentos locais tem estreita relação com o método multigrid e o seu desempenho. Observe que os pontos que resultam da restrição de um refinamento local, no método multigrid, coincidem com pontos da malha imediatamente mais grossa.

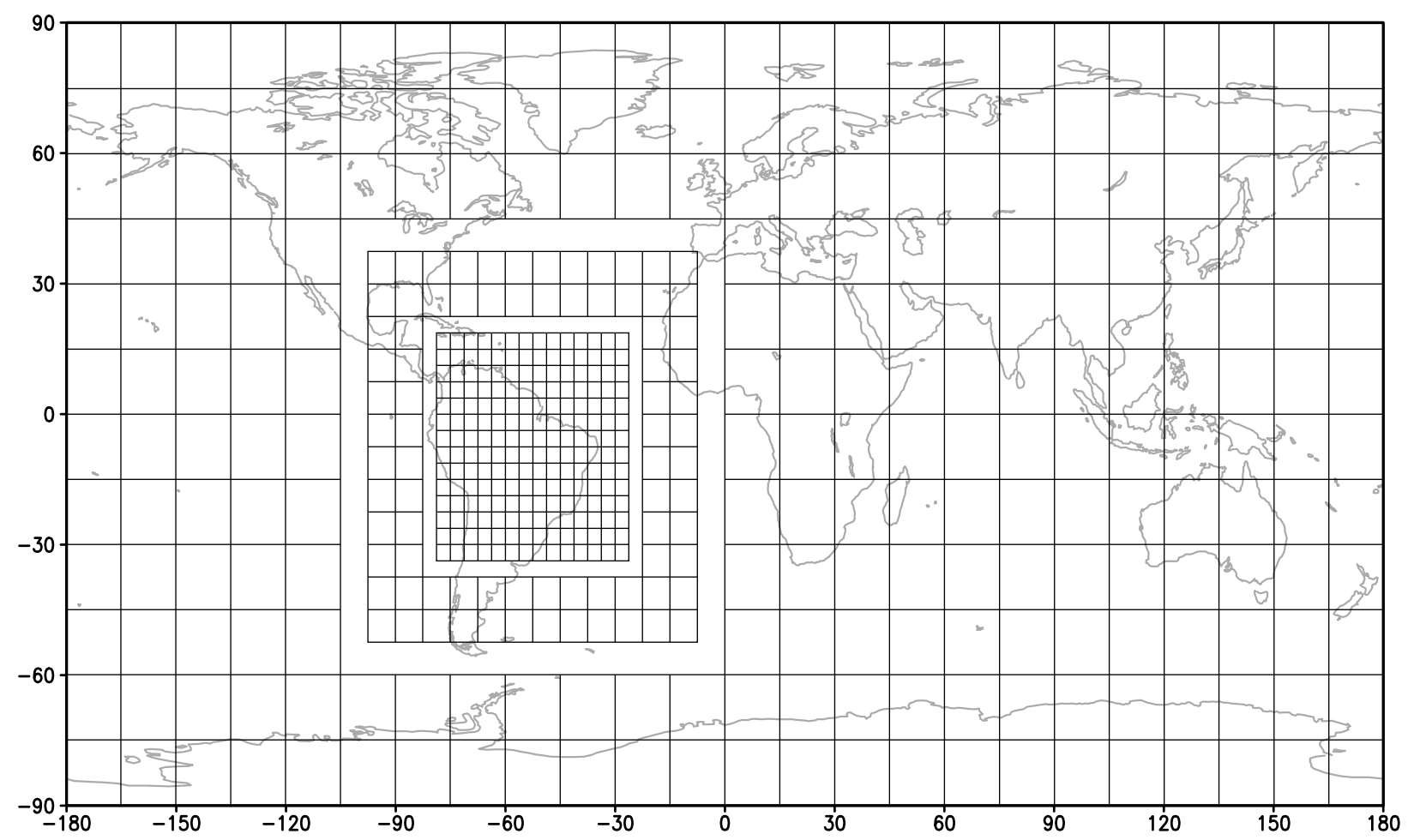

Figura 1.5: Malha base com dois niveis de refinamento. 
Em [28] e [15], cada malha possui adicionalmente duas linhas extras em cada direção, chamadas de pontos fantasmas, como podemos observar na Figura 1.6. Nestes pontos não é calculada a solução e são utilizados para armazenar valores interpolados de malhas de níveis mais grossos/finos, necessários para o cálculo de diferenças finitas no nível atual de refinamento. No entanto, neste trabalho, devido a restrições impostas pelo processo de paralelização, em geral não será possível manter essas duas linhas/colunas adicionais. Isto será tratado com maior detalhe no seguinte capítulo.

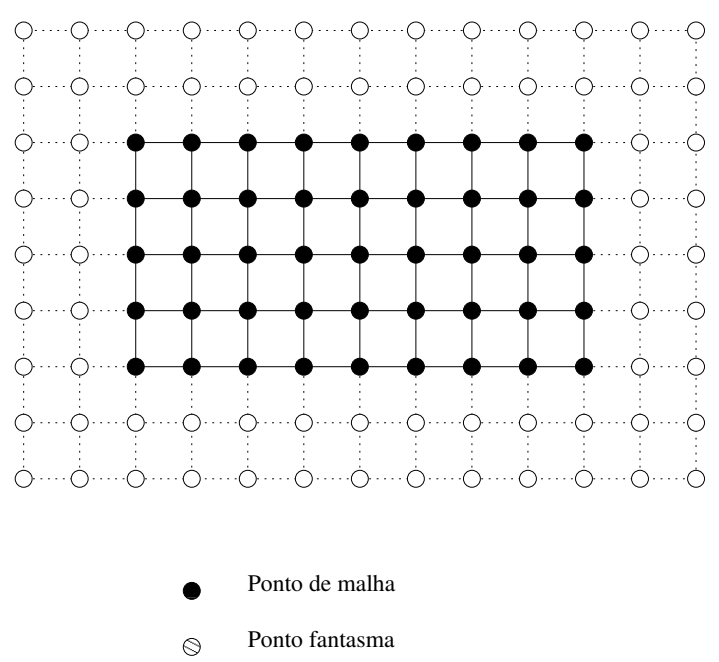

Figura 1.6: Malha refinada com pontos fantasmas. 


\section{Capítulo 2}

\section{Estratégia de paralelização}

Neste capítulo apresentamos a parte central deste trabalho, a qual é nossa estratégia de paralelização do modelo global em diferenças finitas com refinamento local aplicado em máquinas com memória distribuída e empregando passagem de mensagens. Embora a estrutura teórica do mesmo siga [16] e [15], a nossa implementação, realizada seguindo o padrão Fortran 90, é completamente independente. Nosso enfoque será centrado em obter uma distribuição balanceada do trabalho computacional entre os processadores, explorando as independências computacionais naturalmente disponíveis.

Em todo modelo de previsão do tempo podemos distinguir duas grandes componentes: a dinâmica e a física. Na dinâmica, as equações diferenciais parciais que descrevem o movimento de um fluido são integradas, e em modelos baseados em diferenças finitas existe apenas um acoplamento local com valores em um ponto de malha dependendo apenas de valores em pontos de malha em uma vizinhança. Já na física, há apenas um acoplamento vertical devido às rotinas de parametrizações dos fenômenos físicos, tais como: radiação, precipitação, evaporação, outros. Para que se tenha independência entre os processadores durante esta fase, é necessário que cada coluna vertical das variáveis correspondendo a um mesmo ponto de malha seja inteiramente alocada a um único processador. Por este motivo, trabalhamos em decompor horizontalmente o domínio, mantendo a direção vertical intacta.

Por outro lado, nosso modelo possui malhas localmente refinadas, o que dificulta o processo de divisão horizontal devido à mudança na resolução das mesmas. Do capítulo anterior, lembramos que surgem naturalmente três regiões: uma grossa, uma fina e uma de transição, como podemos observar na Figura 2.1, em que a grande maioria dos pontos concentram-se nas malhas grossa e fina. Notamos também que dividir a malha fina, a qual possui uma resolução uniforme, é uma tarefa que não demanda muito esforço. O mesmo para a malha grossa, com uma pequena dificuldade na divisão em uma região vazia em seu interior na qual é inserida a zona de transição. A dificuldade na divisão dessa zona de transição é devido ao fato de termos um conjunto de malhas com resoluções distintas, em que cada uma delas possui uma região vazia em seu interior e uma quantidade pequena de pontos. como pode ser visto na figura abaixo. 

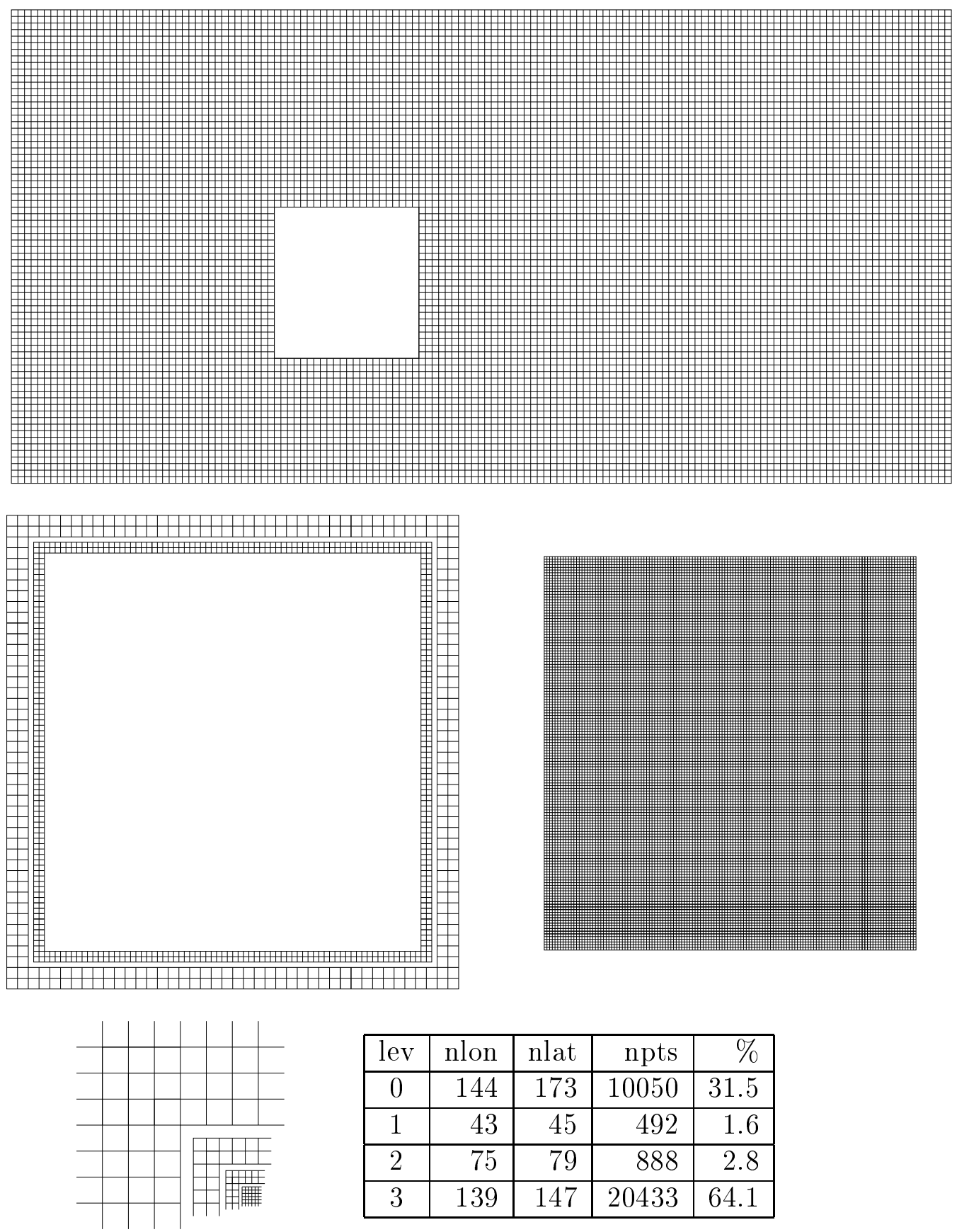

\begin{tabular}{|c|r|r|r|r|}
\hline lev & nlon & nlat & npts & $\%$ \\
\hline 0 & 144 & 173 & 10050 & 31.5 \\
\hline 1 & 43 & 45 & 492 & 1.6 \\
\hline 2 & 75 & 79 & 888 & 2.8 \\
\hline 3 & 139 & 147 & 20433 & 64.1 \\
\hline
\end{tabular}

Figura 2.1: Regióes grossa, de transição e fina para a tabela dada.

Dando prioridade à divisão das malhas grossa e fina, vamos adicionalmente dividir cada uma destas regiões de forma independente, enquanto que para dividir a zona de transição consideramos esta como um único bloco e efetuamos a sua divisão entre o número de processadores determinados para esta região.

No Capítulo 1 afirmamos que para a discretização horizontal utilizamos uma malha do tipo $\mathrm{C}$ de Arakawa [8], que define os campos escalares nos vértices da malha e que chamamos de malha $F$, a componente zonal do vento deslocada meia célula à direita e que chamamos de malha $U$, e a componente meridional do vento deslocada meia célula para cima denominada de malha $V$. Descreveremos brevemente como definimos as malhas $\mathrm{U}$ e $\mathrm{V}$ a partir da malha F. O modelo lê as dimensões das malhas F de um arquivo de configurações 
chamado model. par:

! Number of refinement levels

nref $=2$

! Grids locations

imin_ref $=0,30,3,3,3$

imax_ref $=127,90,117,225,441$

jmin_ref $=0,33,3,3,3$

jmax_ref $=64,61, \quad 53, \quad 97,185$

Figura 2.2: Dimensões das malhas no arquivo de configuração.

No exemplo da Figura 2.2, a variável nref indica que serão considerados dois níveis de refinamento e os vetores imin_ref, imax_ref, jmin_ref e jmax_ref, cujos índices começam em zero, indicam as dimensões da malha base e a posição dos refinamentos locais em relação à malha imediatamente mais grossa. Precisamos indicar que os índices para a malha base começam em zero enquanto para os refinamentos locais começam em um. Logo, para o exemplo acima temos que a malha base (malha de nível 0) possui 128 longitudes (de 0 a 127) e 65 latitudes (de 0 a 64), mas desconsidera a região contida entre as longitudes 30 e 90 e as latitudes 33 e 61, na qual se encontra localizado o primeiro refinamento local (lembre que os refinamentos não encostam na malha grossa onde estão inseridos). Desta forma, o primeiro refinamento local (malha de nível 1) possui $2 \times(90-30)-1=119$ longitudes e $2 \times(61-33)-1=55$ latitudes, mas por sua vez desconsidera a região contida entre as longitudes 3 e 117 e latitudes 3 e 53 , em que temos localizado o segundo refinamento local (malha de nível 2), possuindo $2 \times(117-3)-1=227$ longitudes e $2 \times(53-3)-1=99$ latitudes. Observe que, mesmo tendo informações para processar quatro níveis de refinamento, somente dois níveis de refinamento serão processados, devido ao valor da variável nref.

Uma vez definidas as malhas $\mathrm{F}$, em função destas definimos as malhas $\mathrm{U}$ e as malhas $\mathrm{V}$. Como citado anteriormente, os pontos das malhas U são definidos deslocando meia célula à direita os pontos das malhas F, mas é necessário efetuar algumas considerações. Primeiro, buscamos uma simetria na fronteira entre duas malhas U. Segundo, impomos que a interpolação cúbica em um ponto de partida durante o transporte Semi-Lagrangiano seja efetuada empregando pontos de malhas de até dois níveis diferentes, como máximo. Fazemos isto para minimizar a comunicação entre os processadores. Terceiro, o vento zonal não está definido nos polos, e na malha global (nível 0) não é necessário definir pontos para a malha U nos polos e, pois embora estejam definidos em nossa implementação, eles não são utilizados. Deste modo, seja $\mathrm{k}<$ nref o nível de uma malha e sejam $I_{1}^{k}, J_{1}^{k}$ e $J_{2}^{k}$ os correspondentes valores de imin_ref $(k+1)$, jmin_ref $(k+1)$ e jmax_ref $(k+1)$. Considerando os pontos da malha $\mathrm{F} F_{i, j}$, com $i=I_{1}^{k}$ e $j=J_{1}^{k}+1, \ldots, J_{2}^{k}-1$, seus correspondentes pontos da malha $\mathrm{U}$ são $U_{i+\frac{1}{2}, j}$, deslocados meia célula à direita. Porém, estes pontos invadem a região da malha mais fina de nível $k+1$ e por conseguinte não são definidos. De forma análoga, considerando 
a malha fina de nível $\mathrm{k}+1$ e supondo que tenha $m$ longitudes e $n$ latitudes, para o ponto da malha $\mathrm{F} F_{m, j}, j=1, \ldots, n$, seus correspondentes pontos da malha $\mathrm{U}$ são $U_{n+\frac{1}{2}, j}$, mas estes pontos estão fora da malha e portanto também não são definidos. A Figura 2.3 mostra a distribuição dos pontos U próximos da fronteira entre duas malhas com as decisões acima mencionadas, garantindo uma simetria entre as bordas esquerda e direita de duas malhas $\mathrm{U}$ e gerando uma distância lateral entre as mesmas de 1.25 vezes o espaçamento longitudinal da malha grossa, mostrado na região sombreada (note que os pontos U do refinamento mais fino não são mostrados). Fazendo considerações análogas para as malhas $\mathrm{V}$ obtemos uma simetria entre as bordas inferior e superior de duas malhas $\mathrm{V}$.

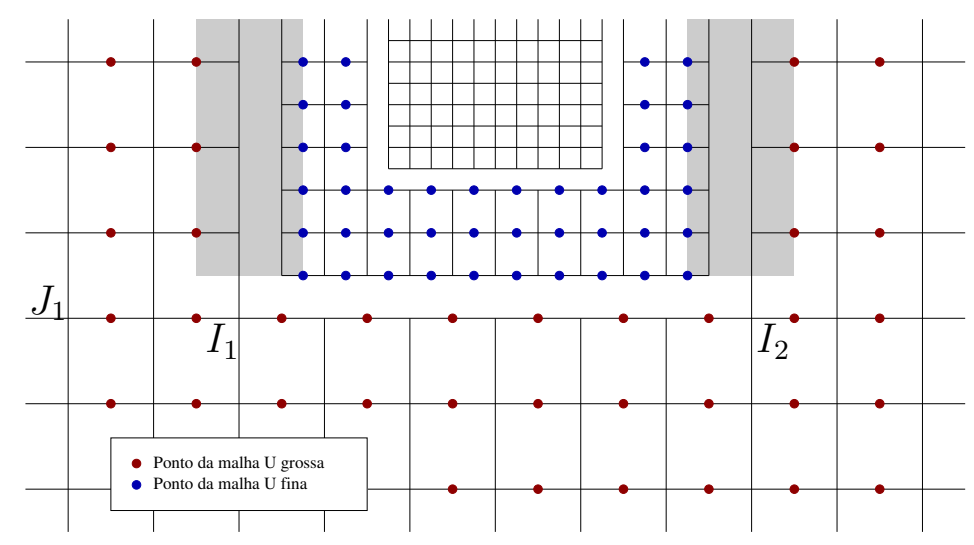

Figura 2.3: Pontos U próximos da fronteira entre duas malhas (versão original).

Durante grande parte do desenvolvimento do modelo as malhas $\mathrm{U}$ e $\mathrm{V}$ foram definidas desta forma. Contudo, durante a etapa de testes do transporte Semi-Lagrangiano verificamos o surgimento de ruído nas fronteiras das malhas que contaminavam rapidamente o interior das mesmas, comprometendo desta forma os resultados. Após testes adicionais e repetidas verificações do código concluimos que este ruído foi causado pela distância entre duas malhas U (ou V), mostrado na Figura 2.3 pela região sombreada. Conseguimos resolver este problema alterando a definição da malha U nos refinamentos locais, compensando a perda dos pontos U na malha grossa, na fronteira com a malha fina, com a adição de pontos U na malha fina que foram gerados deslocando meia célula à esquerda e à direita a primeira e última coluna de pontos $\mathrm{F}$ da malha fina, respectivamente. A Figura 2.4 mostra a nova distribuição dos pontos U próximos da fronteira entre duas malhas. Adicionalmente mostra que a distância lateral entre as mesmas é agora 0.75 vezes o espaçamento longitudinal da malha grossa. Alterações análogas foram feitas para a malha $\mathrm{V}$. 


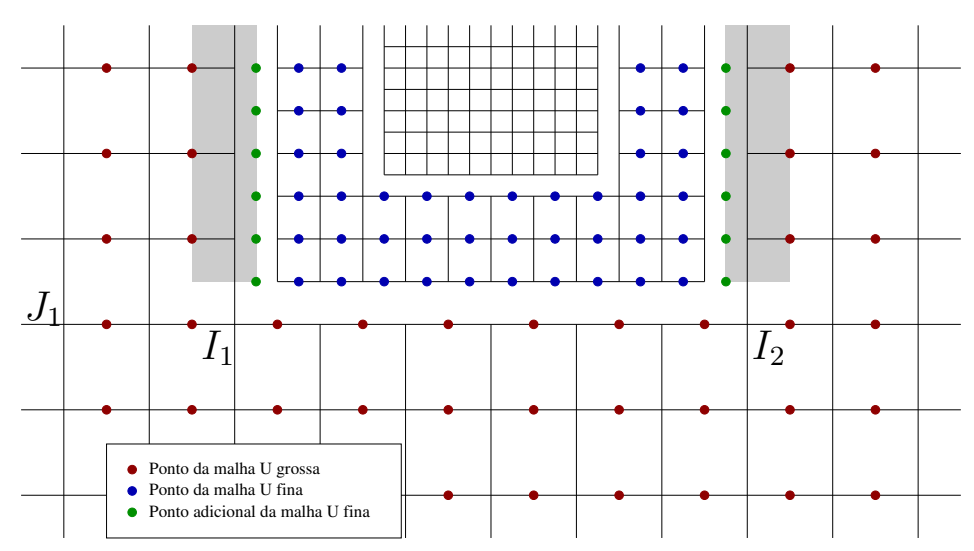

Figura 2.4: Pontos U próximos da fronteira entre duas malhas (versão final).

\section{$2.1 \quad$ Faixas e remendos}

Supondo que temos uma única malha para dividir entre um certo número de processadores, talvez a forma mais simples de fazer isto seja dividindo primeiro a malha, digamos horizontalmente, em faixas ou strips, e em seguida dividir, agora verticalmente, cada uma destas faixas em remendos ou patches. Inicialmente podemos considerar que cada uma destas faixas tem um número completo de linhas e que cada remendo (dentro da faixa) tem um número completo de colunas (ver Figura 2.5).

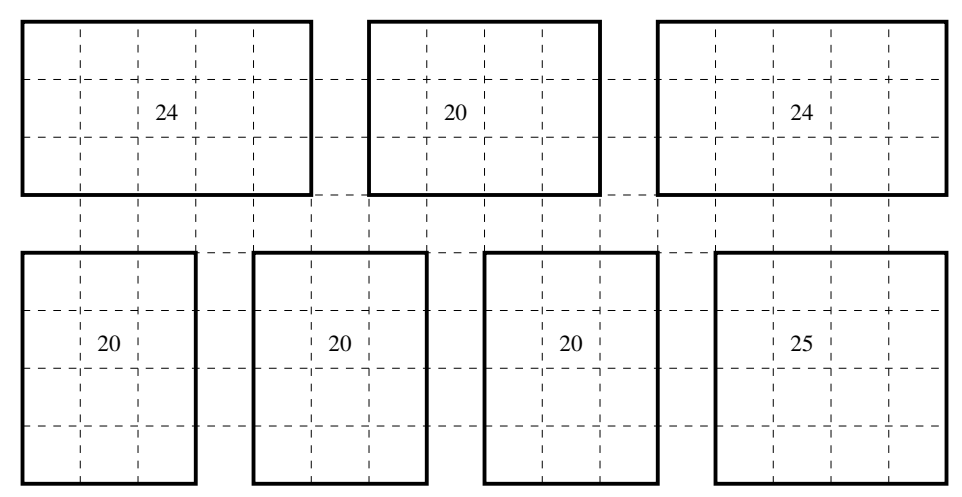

Figura 2.5: Remendos com linhas/colunas completas.

Aprimorando o procedimento descrito acima, podemos permitir que parte de uma linha pertença a uma faixa e o restante dela à faixa seguinte. De forma similar, dentro de cada faixa, permitimos que parte de uma coluna pertença a um remendo e o restante dela ao remendo seguinte. Desta forma, teríamos uma divisão mais balanceada da malha, no sentido em que a diferença entre o número de pontos de cada remendo tem como máximo um (ver Figura 2.6). Disto surgem algumas questões, como por exemplo: 
- Quantas faixas devemos considerar?

- Em quantos remendos devemos dividir cada faixa?

- Como representamos faixas/remendos com linhas/colunas incompletas?

- Se a malha tiver uma região vazia no interior, como ficam as faixas/remendos?

Portanto, essa é a nossa estratégia de divisão de malhas, isto é, primeiro dividimos a malha horizontalmente em faixas e em seguida dividimos verticalmente cada faixa em remendos. As questões levantadas serão respondidas ao longo das seguintes seções.

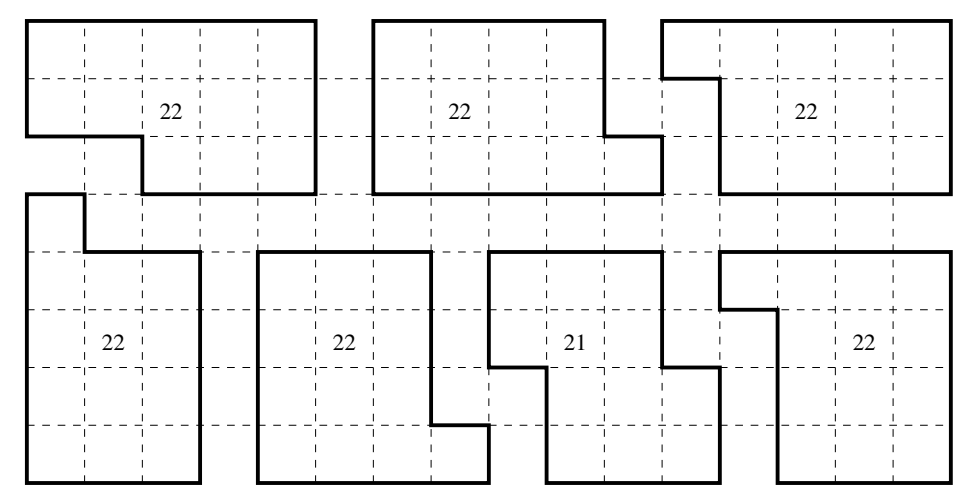

Figura 2.6: Remendos com quebra de linhas/colunas.

\subsection{Representação de malhas irregulares}

Agora, precisamos desenvolver uma estrutura de dados que nos permita descrever, tanto zonal como meridionalmente, malhas (logicamente) irregulares (Figura 2.7), embora na implementação cada malha seja um arranjo bi(tri)-dimensional. Isto se torna necessário pois em nossa estratégia permitimos que uma malha tenha linhas (latitudes) ou colunas (longitudes) incompletas ou, ainda mais, consideramos a possibilidade da malha ter uma região vazia no seu interior.

Em [57] é apresentada uma estrutura de dados para a manipulação de malhas escalonadas (stair-step grid) na resolução de equações diferenciais parciais hiperbólicas utilizando malhas adaptativas compostas (CAG). Esta estrutura, implementada em $\mathrm{C} / \mathrm{C}++$, consiste de uma lista ligada de segmentos de dados, cada um destes contendo os índices iniciais e finais da coluna do segmento, um apontador ao vetor de dados e um apontador ao seguinte segmento na mesma fila. Deste trabalho aproveitamos a forma de representar uma malha, isto é, por segmentos e, considerando também métodos para representar matrizes esparsas CRS/CCS (Compressed Row/Column Storage) [12], desenvolvemos o nosso descritor de malhas irregulares, com o objetivo de realizar a varredura de uma malha (logicamente) irregular, por segmentos, sejam estes linhas ou colunas. Para isto, nosso descritor consiste basicamente de 


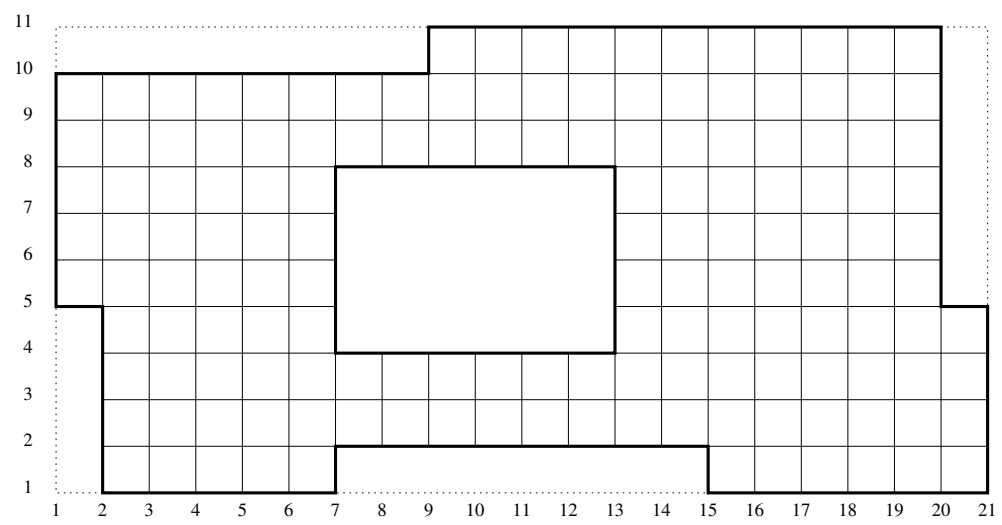

Figura 2.7: Malha (logicamente) irregular.

um número e três vetores. O número indica quantos segmentos tem a malha, dois vetores servem para guardar as posições iniciais e finais das longitudes (latitudes) de cada segmento e o terceiro guarda as latitudes (longitudes) dos mesmos. Para completar o descritor, temos variáveis que indicam o número total de pontos e a caixa onde está inserida a malha, como mostrado abaixo:

! GRID_DESC definition

type grid_desc

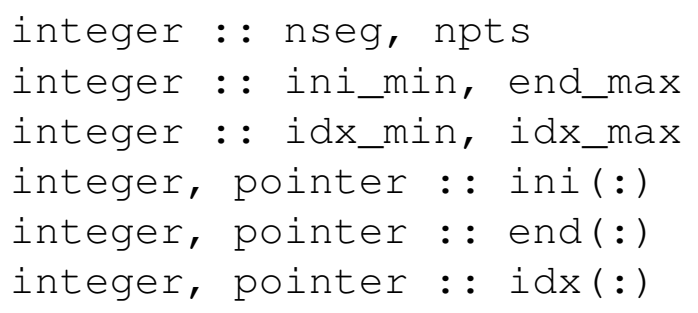

Figura 2.8: Estrutura do descritor de malhas irregulares.

Assim, tomando como exemplo a Figura 2.7, temos que a Figura 2.9 mostra os valores do descritor zonal, enquanto a Figura 2.10 mostra os valores correspondentes ao descritor meridional.

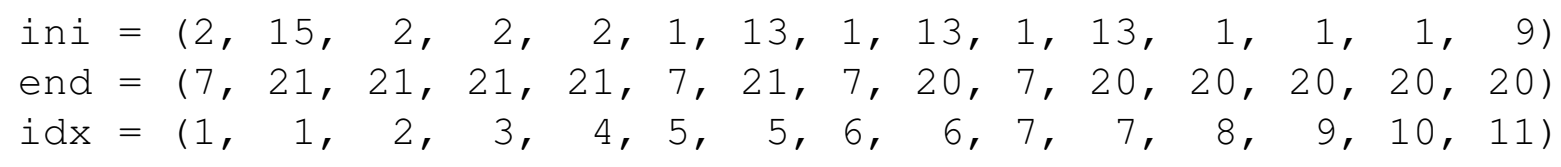

Figura 2.9: Descritor zonal para a Figura 2.7. 


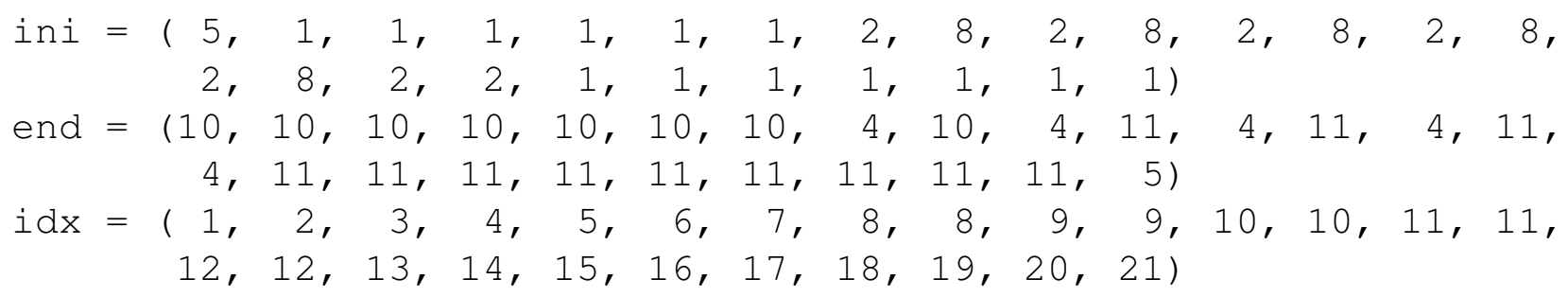

Figura 2.10: Descritor meridional para a Figura 2.7.

Uma vez que o descritor é criado, podemos processar a malha por segmentos, da seguinte forma:

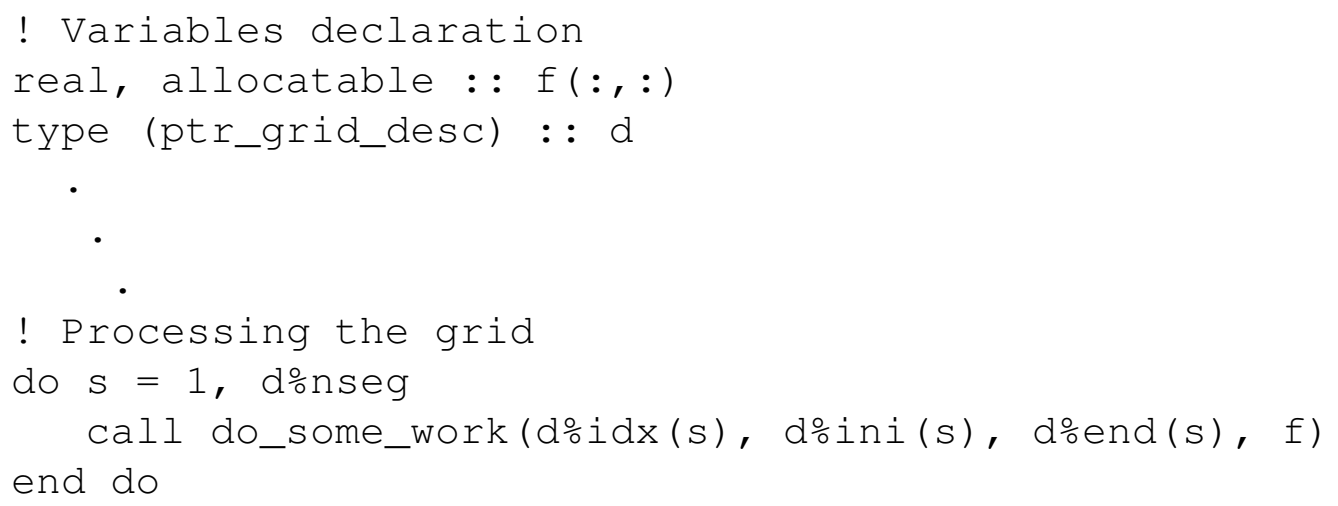

Figura 2.11: Varredura de uma malha irregular por segmentos.

\subsection{Definição dos campos}

Nosso modelo possui malhas localmente refinadas, pelo que faz-se necessário ter alocados os diversos campos em níveis diferentes. A forma tradicional de se fazer isto em FORTRAN 77 é alocando um vetor contendo todos os campos e acessando eles por indexação indireta. No entanto, novas características no padrão Fortran 90, como módulos, tipos derivados e apontadores, permitem uma implementação mais clara e segura, onde cada aspecto do modelo pode ser representado utilizando abstrações familiares. Em [11] é desenvolvida uma estrutura de dados, em Fortran 90 e OpenMP [7], para a manipulação de malhas com refinamentos adaptativo (AMR) na resolução de problemas elíptivos e hiperbólicos em máquinas com memória distribuída compartilhada (DSM). Esta estrutura consiste de um tipo derivado encapsulando a informação sobre as dimensões e localização da malha assim como apontadores aos campos, dinâmicamente alocados. Embora não necessitemos criar e eliminar dinâmicamente malhas, deste trabalho aproveitamos a ideia de definir os campos como apontadores a arranjos bi(tri)-dimensionais alocados dinâmicamente. Ainda mais, desde que o nosso modelo pode ter vários níveis de refinamento e que nem todos eles serão empregados por todos os processadores, definimos cada campo como um vetor de apontadores a arranjos bi(tri)-dimensionais e adicionalmente definimos um vetor de inteiros 
para indicar quais níveis estão ativos (valor positivo) ou inativos (valor zero) no processador. A estrutura de dados para um campo tridimensional é mostrada na seguinte figura:

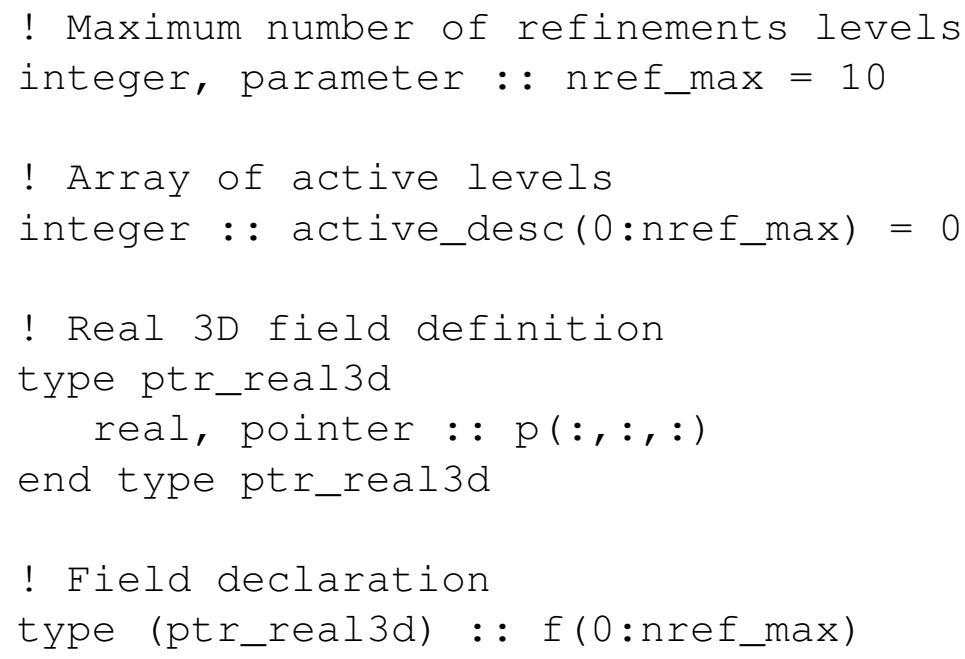

Figura 2.12: Estrutura de dados para um campo tridimensional.

Considerando estas novas estruturas, a Figura 2.13 mostra a varredura de um conjunto de malhas, em diferentes níveis, por segmentos (note a semelhança com a Figura 2.11):

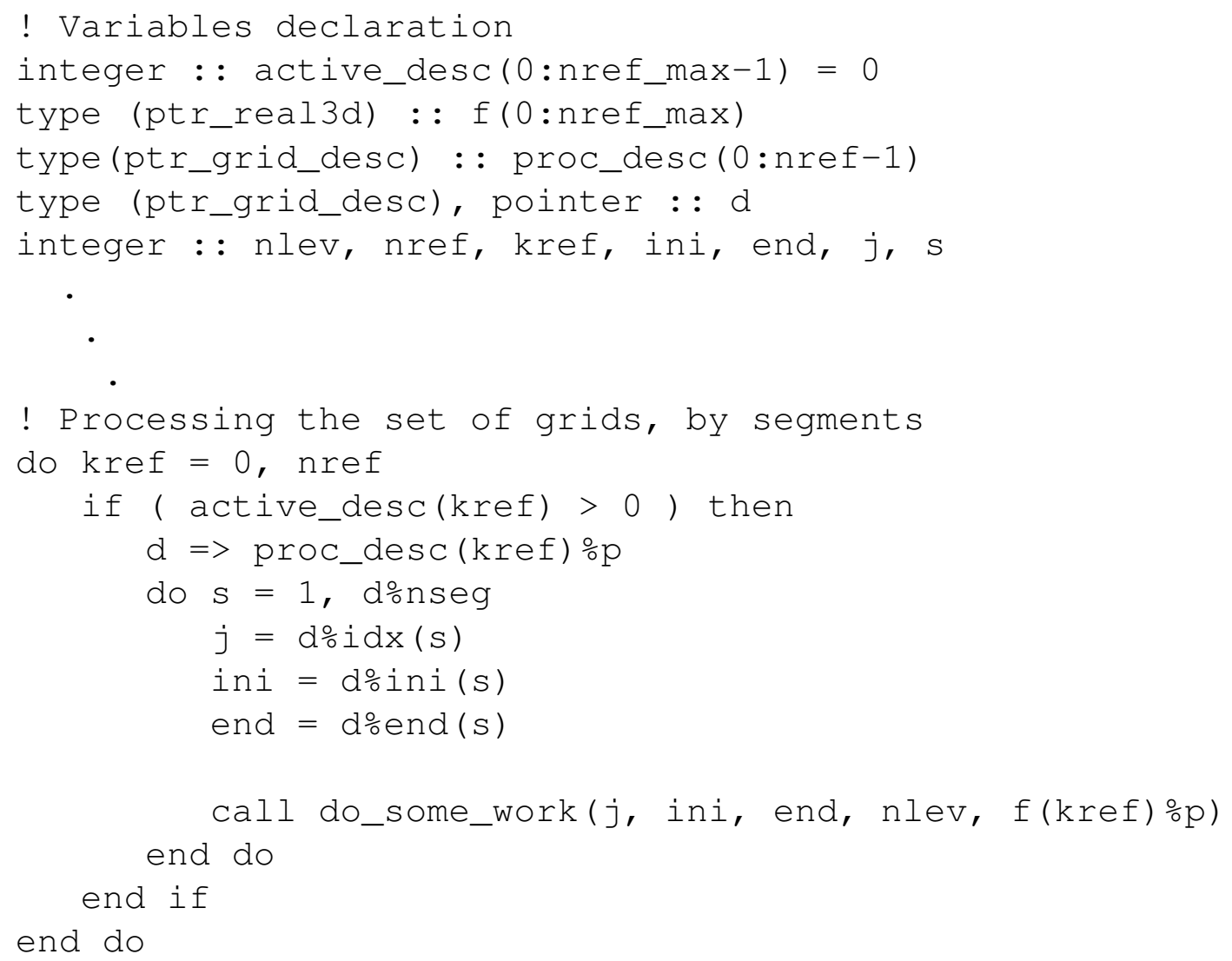

Figura 2.13: Varredura de um conjunto de malhas irregulares por segmentos. 


\subsection{Decomposição do domínio}

Uma vez obtida a representação de malhas irregulares, tanto no sentido zonal como meridional, iniciamos o processo de decomposição bidimensional do domínio, lembrando que cada uma das regiões (grossa, fina e de transição) será dividida de forma independente entre um número de processadores determinado de forma proporcional ao número de pontos destas, sendo o processo para as malhas grossa e fina praticamente igual. Nossa estratégia resume-se em dividir uma malha em faixas (strips) na direção longitudinal e posteriormente dividir cada faixa em remendos (patches) na direção meridional.

Determinamos o número de faixas e de processadores por faixa em que uma malha será dividida, efetuando primeiro uma decomposição contínua da mesma, calculando a sua área (considerando uma esfera unitária) e em seguida dividindo este valor entre o número de processadores, obtendo assim a área correspondente a um processador. Atribuindo a cada um dos processadores uma parcela da malha com esta área, da forma mais quadrada possível, obtemos o número de faixas e de processadores em cada uma destas. Uma vez determinados estes valores, os utilizamos na decomposição discreta da malha, para o qual calculamos o número de pontos da malha, o número de pontos por processador e o número de pontos para cada faixa, de forma proporcional ao número de processadores que cada uma destas contém. Antes de proceder com a divisão das faixas, no sentido meridional, necessitamos de uma estrutura que nos permita diferenciar elas dentro da malha. Em [36] é desenvolvida a biblioteca RSL (Runtime System Library), para ser implementada com modelos de previsão do tempo, utilizando malhas aninhadas regulares (retângulos ou a união deles), em máquinas paralelas com memória distribuida. Nele, é empregada uma matriz de inteiros para mostrar, no texto, a decomposição horizontal de uma malha. Aproveitamos esta ideia para definir o mapa de distribuição de faixas/processadores como uma matriz de inteiros em que o valor em cada ponto indica o número de faixa/processador a que o mesmo pertence. Uma vez calculados os números de pontos das faixas (regiões da malha não consideradas por ser irregular ou por conter regiões vazias no seu interior são previamente marcadas no mapa com um valor especial, normalmente negativo) as escrevemos no mapa na direção longitudinal, para em seguida calcular o número de pontos para cada processador dentro de cada faixa e substituir, por meio de uma varredura meridional, o valor desta no mapa pelo número do respectivo processador. Cada processador armazena um cópia deste mapa.

\subsubsection{Decomposição da malha fina}

Começamos dividindo a malha fina por ser uma divisão mais simples. Se a malha fina é determinada pelas longitudes $\lambda_{1}$ e $\lambda_{2}$ e pelas latitudes $\theta_{1}$ e $\theta_{2}$ então a área desta região, considerando uma esfera unitária, é dada por

$$
s=\left(\lambda_{2}-\lambda_{1}\right)\left(\sin \left(\theta_{2}\right)-\sin \left(\theta_{1}\right)\right) .
$$


Surgindo a necessidade de dividir esta região em $n$ processadores, a área correspondente a cada um destes será

$$
s_{1}=s / n
$$

Criamos agora um vetor auxiliar $v$ com $n+1$ entradas tendo como valores as latitudes correspondentes a sucessivos incrementos de $s_{1}$, isto é,

$$
v(k+1):=\theta_{k}=\arcsin \left(\frac{k \times s_{1}}{\lambda_{2}-\lambda_{1}}+\sin \left(\theta_{1}\right)\right), \quad k=0, \ldots, n .
$$

Utilizando os valores do vetor $v$ construímos as faixas. Mostramos, como exemplo, a construção da primeira delas. Seja $p$ um número natural, observe que a região delimitada pelas longitudes $\lambda_{1}$ e $\lambda_{2}$ e pelas latitudes $v(1)$ e $v(1+p)$ possui uma área igual a $p \times s_{1}$, isto é, a área correspondente a $p$ processadores, mais ainda, a altura desta região é igual a $v(1+p)-v(1)$ e a largura media é igual a $\left(\lambda_{2}-\lambda_{1}\right) \cos \left(\theta_{m}\right)$, em que $\theta_{m}$ é uma latitude média obtida a partir de $v(1)$ e $v(1+p)$ e que neste trabalho é considerada como a latitude que divide a área da região pela metade,

$$
\theta_{m}=\arcsin \left(\frac{\sin (v(1))+\sin (v(1+p))}{2}\right) .
$$

Lembrando que a região em que estamos trabalhando corresponde à área de $p$ processadores, definimos a razão entre a largura e a altura da mesma por

$$
r(p)=\frac{\left(\lambda_{2}-\lambda_{1}\right) \cos \left(\theta_{m}\right)}{p(v(1+p)-v(1))}
$$

Assim, a ideia é começar com $p=1$, calcular $\theta_{m}$ e $r(1)$ e em seguida avaliar se esta razão é menor ou igual a uma razão padrão $r_{0}$. Em tal caso, teremos definido nossa primeira faixa, em caso negativo aumentamos gradativamente $p=2,3, \ldots$ até $r(p)$ ser menor ou igual a $r_{0}$. Neste trabalho, devido a que a latitude média $\theta_{m}$ da faixa e consequentemente sua largura média variam rapidamente conforme varia o número de processadores na mesma, principalmente em regiões próximas aos polos, consideramos $r_{0}=3$.

Todas as faixas são criadas utilizando o procedimento anterior, mas devemos fazer a seguinte observação: cada faixa ímpar é construída no sentido sul-norte, enquanto cada faixa par é construída no sentido norte-sul. Por exemplo, para construir a segunda faixa procuramos um número natural $q$ tal que a razão entre a largura e a altura da região delimitada pelas longitudes $\lambda_{1}$ e $\lambda_{2}$ e pelas latitudes $v(n+1-q)$ e $v(n+1)$ seja menor ou igual a $r_{0}$. Uma vez construídas todas as faixas e determinado o número de processadores para cada uma delas efetuamos um processo de correção de faixas, pois pode ocorrer que a última faixa tenha um número muito pequeno de processadores. Se este número é muito pequeno, então, estes processadores são alocados às faixas vizinhas e a última faixa é eliminada. Caso contrário, processadores de faixas vizinhas são alocados nesta última faixa até conseguirmos 
que a mesma tenha um número de processadores próximo aos dos seus vizinhos. Terminado este processo, as faixas são reordenadas para estarem no sentido sul-norte.

Conhecendo o número de faixas e de processadores para cada uma delas, podemos calcular com exatidão o número de pontos que cada faixa conterá, e dentro de cada uma delas, o número de pontos contidos em cada remendo. Uma vez determinados estes valores, escrevemos (horizontalmente) no mapa um valor identificando a faixa a que corresponde cada ponto do mesmo, e em seguida, dentro de cada faixa, escrevemos (verticalmente) o número do remendo a qual tal ponto pertence.

Após dividir a malha em remendos e gerar o mapa de distribuição, podemos extrair a partir deste último e utilizando o descritor de malhas descrito anteriormente, o descritor para cada um destes remendos e os armazenar em um repositório de malhas.
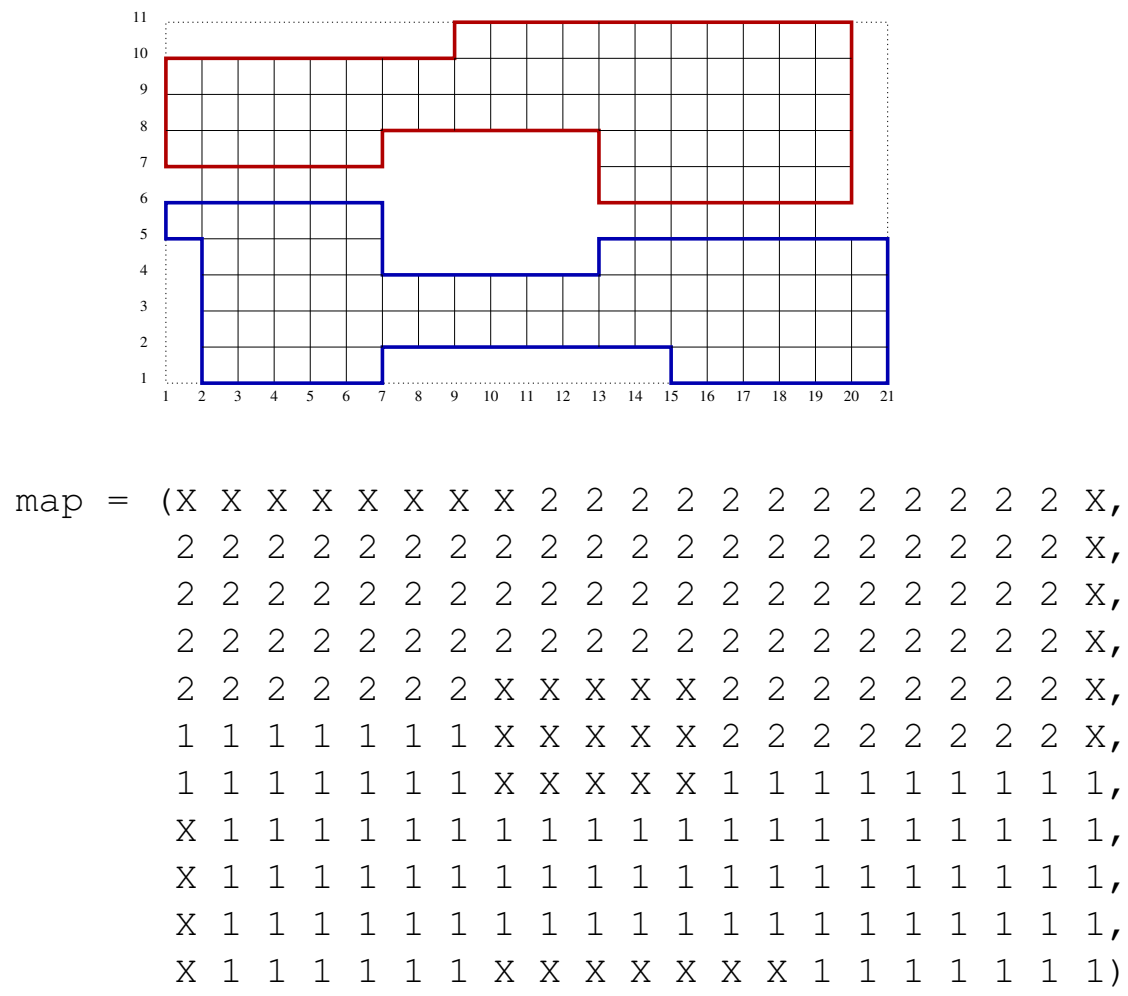

Figura 2.14: Divisão de uma malha irregular em faixas e conteúdo do mapa $(X<0)$.

\subsubsection{Decomposição da malha grossa}

Como dito anteriormente, o processo de divisão da malha grossa é praticamente igual ao da malha fina, mas com algumas diferenças. Primeiro, no momento de construir o vetor auxiliar de latitudes, as faixas e os remendos devemos considerar que temos uma região vazia onde estão inseridas a região de transição e a malha fina; segundo, devido à periodicidade da esfera, surge a possibilidade de desalinhar as faixas em relação à primeira coluna da malha com o intuito de minimizar a comunicação entre os processadores e por fim, devido à esfericidade da Terra, os remendos das faixas que contém os polos não são quadrados, o que 
nos faz considerar formas alternativas de decomposição da malha grossa.

Em relação à termos uma malha com uma região vazia, modificamos convenientemente as fórmulas para calcular o vetor auxiliar de latitudes. Uma vez determinado o número de faixas, o número de remendos por faixa e criado o mapa, marcamos neste último a região correspondente à zona de transição e malha fina com um valor especial, normalmente negativo, para que no momento de escrever as faixas e os remendos nele saibamos que não devemos considerar esta região. Já para o desalinhamento das faixas estabelecemos algumas condições: primeiro, toda faixa contendo um remendo não é desalinhada; segundo, de forma geral intercalamos o desalinhamento das faixas, isto é, uma sim, uma não; terceiro, o desalinhamento de faixas com mais de um remendo que estejam abaixo ou acima da região de transição corresponde à metade da largura de um remendo e por fim, faixas com mais de um remendo contendo parcial ou totalmente a região de transição são alinhadas com a coluna que divide, verticalmente, esta última pela metade.

\section{Decomposição IGLOO}

Como mencionamos anteriormente, devido à esfericidade da Terra, observamos que os remendos das faixas que contém os polos não são quadrados (Figura 2.23). Assim,devemos modificar nossa estratégia de decomposição da malha grossa para uma outra que considere este fato. Adotamos a técnica IGLOO, desenvolvida em [14], que consiste em definir uma calota polar, que é alocada a um único processador e que o número de remendos por faixa aumenta conforme a faixa esteja mais próxima do equador. No entanto, o uso desta técnica nos leva a uma restrição: cada calota polar deve ter como minimo um número de pontos igual a duas latitudes completas. Na Figura 2.24 podemos ver a aplicação desta técnica. Compare também as figuras 2.21 e 2.22 .

\subsubsection{Decomposição da zona de transição}

A divisão da zona de transição é semelhante, com algumas modificações. Primeiro, a zona é considerada como um único bloco, segundo; não é permitido que pontos de uma mesma linha (latitude) pertençam a duas faixas diferentes, terceiro; não é permitido que dentro de uma faixa, pontos de uma mesma coluna (longitude) pertençam a remendos diferentes e por fim, o algoritmo para determinar o número de faixas e de remendos por faixas deve evitar que um remendo tenha duas regiões disjuntas.

A seguir apresentamos três exemplos de divisão de malhas para uma mesma configuração, fornecida pela tabela abaixo:

A Tabela 2.1 mostra o número de pontos por região, o número de pontos por processador dentro de cada região e a porcentagem do desbalanço no número de pontos em relação ao caso ideal, mostrado na última linha, para uma divisão entre 20 processadores. Valores negativos para este desbalanço indicam um número menor de pontos por processador do que no caso ideal, enquanto valores positivos indicam um número maior de pontos por 
nref $=4$

imin_ref $=0,102, \quad 3,3,3$

imax_ref $=359,148, \quad 89,169,329$

jmin_ref $=0, \quad 52, \quad 3, \quad 3, \quad 3$

jmax_ref $=180,103, \quad 99,189,369$

Figura 2.15: Dados para uma malha grossa com 4 refinamentos locais.

processador. Os dados correspondem aos valores da Figura 2.15. As figuras 2.16 e 2.17 mostram a decomposição obtida para as malhas grossa e fina.

\begin{tabular}{|c|c|c|c|c|c|}
\hline região & npts & $\%$ & nproc/reg & npts/proc & $\%$ des \\
\hline grossa & 62910 & 11.52 & 3 & 20970 & -23.22 \\
\hline trans & 7428 & 1.36 & 1 & 7428 & -72.80 \\
\hline fina & 475881 & 87.12 & 16 & 29743 & 8.90 \\
\hline \hline (ideal) & 546219 & 100.00 & 20 & 27311 & - \\
\hline
\end{tabular}

Tabela 2.1: Pontos por processador para cada região e para o caso ideal, para 20 processadores.

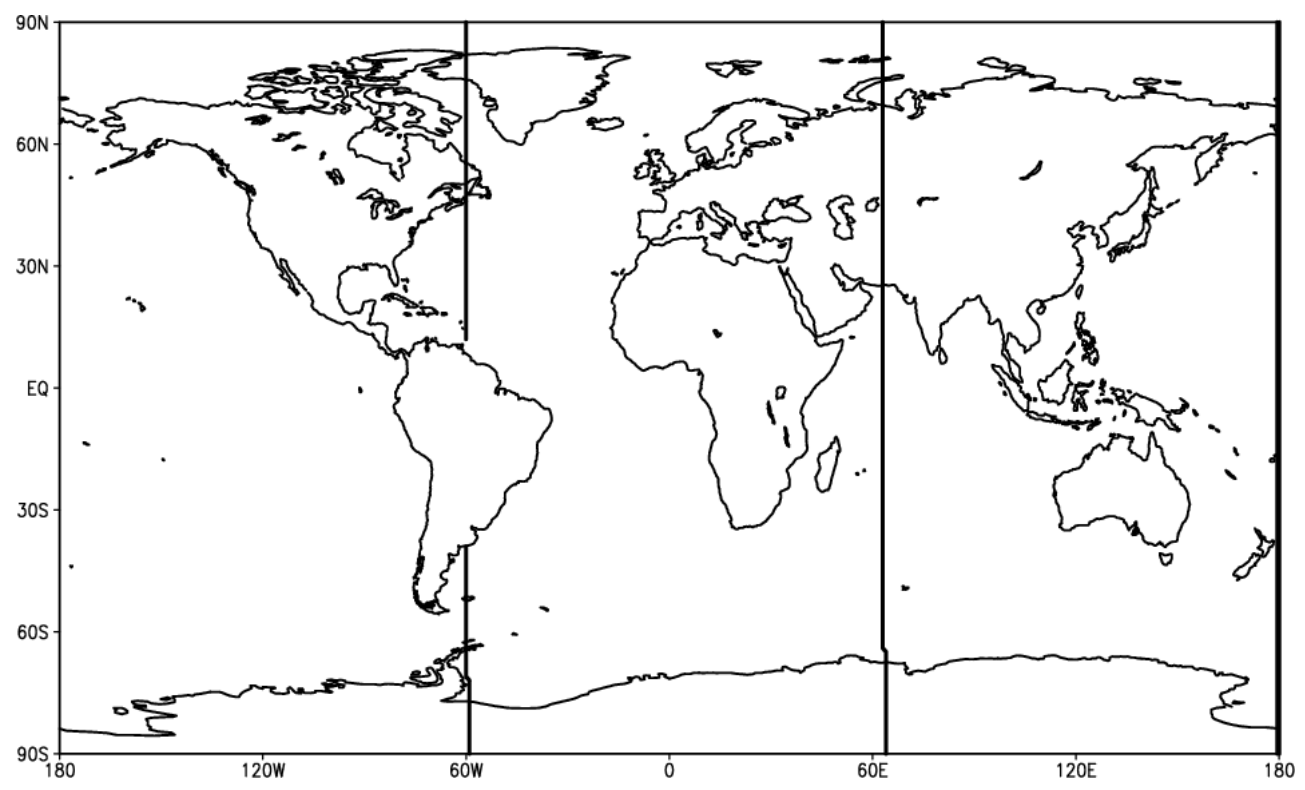

Figura 2.16: Decomposição da malha grossa para os dados da Tabela 2.1.

De forma similar, as figuras 2.18 e 2.19 mostram a decomposição obtida para as malhas grossa e fina, para os dados da Tabela 2.2, correspondentes a uma divisão entre 79 processadores. E a Figura 2.20 mostra a decomposição total do conjunto de malhas. 


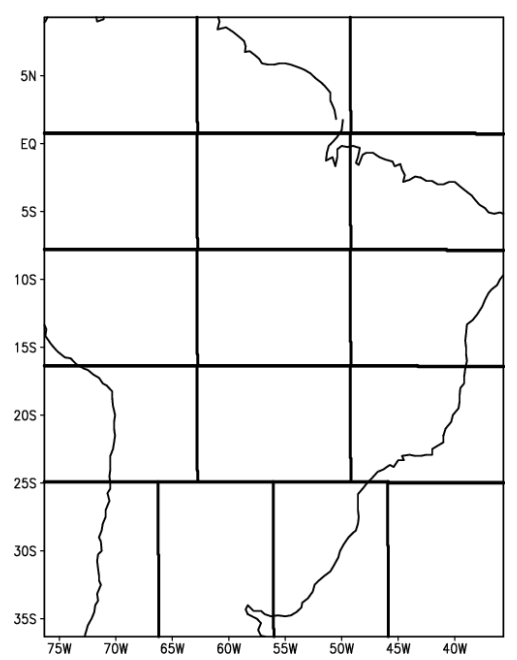

Figura 2.17: Decomposição da malha fina para os dados da Tabela 2.1.

\begin{tabular}{|c|c|c|c|c|c|}
\hline região & npts & $\%$ & $\mathrm{np} / \mathrm{reg}$ & $\mathrm{npts} / \mathrm{pr}$ & $\%$ des \\
\hline grossa & 62910 & 11.52 & 9 & 6990 & 1.08 \\
\hline trans & 7428 & 1.36 & 2 & 3714 & -46.29 \\
\hline fina & 475881 & 87.12 & 68 & 6999 & 1.21 \\
\hline \hline (ideal) & 546219 & 100.00 & 79 & 6915 & - \\
\hline
\end{tabular}

Tabela 2.2: Pontos por processador para cada região e para o caso ideal, para 79 processadores.

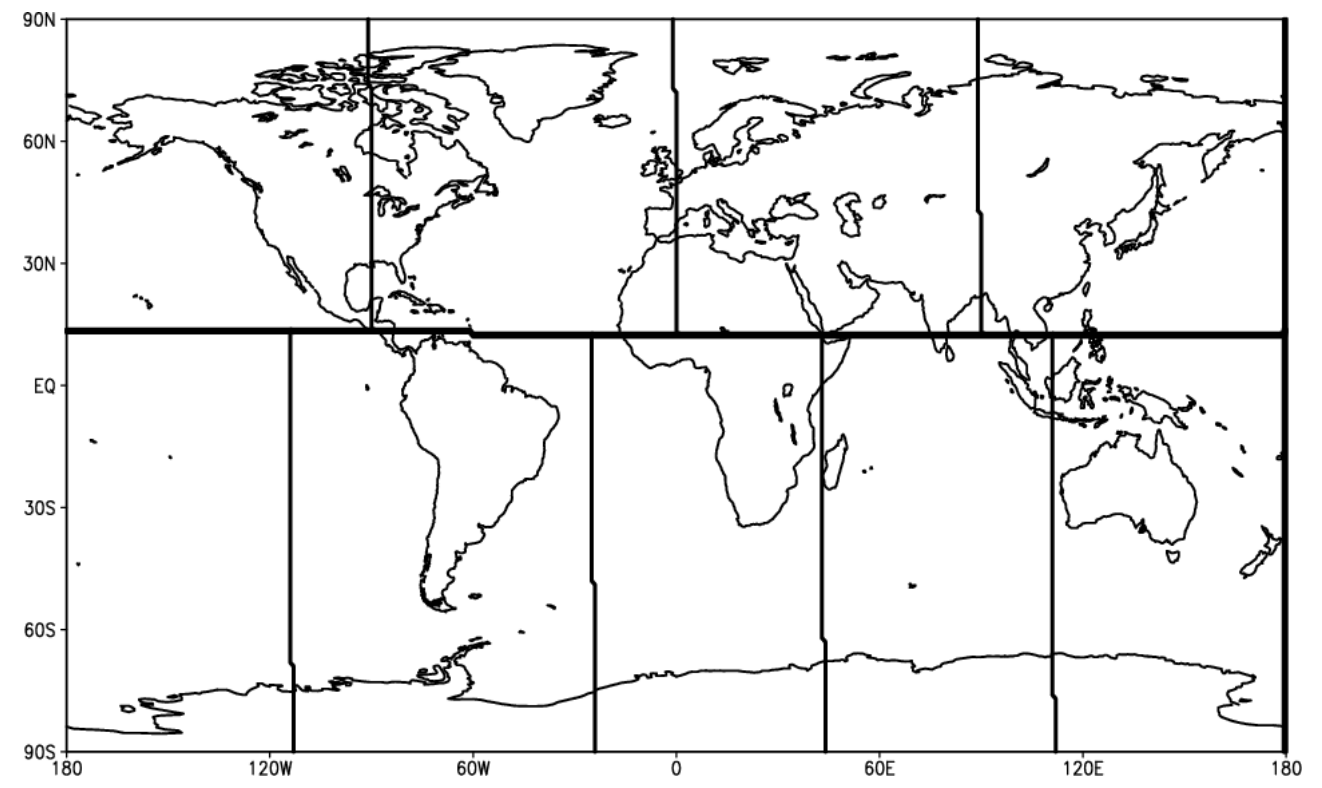

Figura 2.18: Decomposição da malha grossa para os dados da Tabela 2.2. 


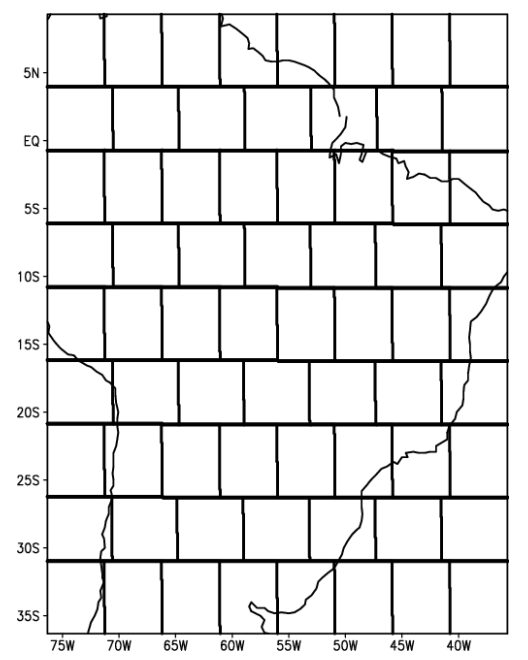

Figura 2.19: Decomposiçẫo da malha fina para os dados da Tabela 2.2.

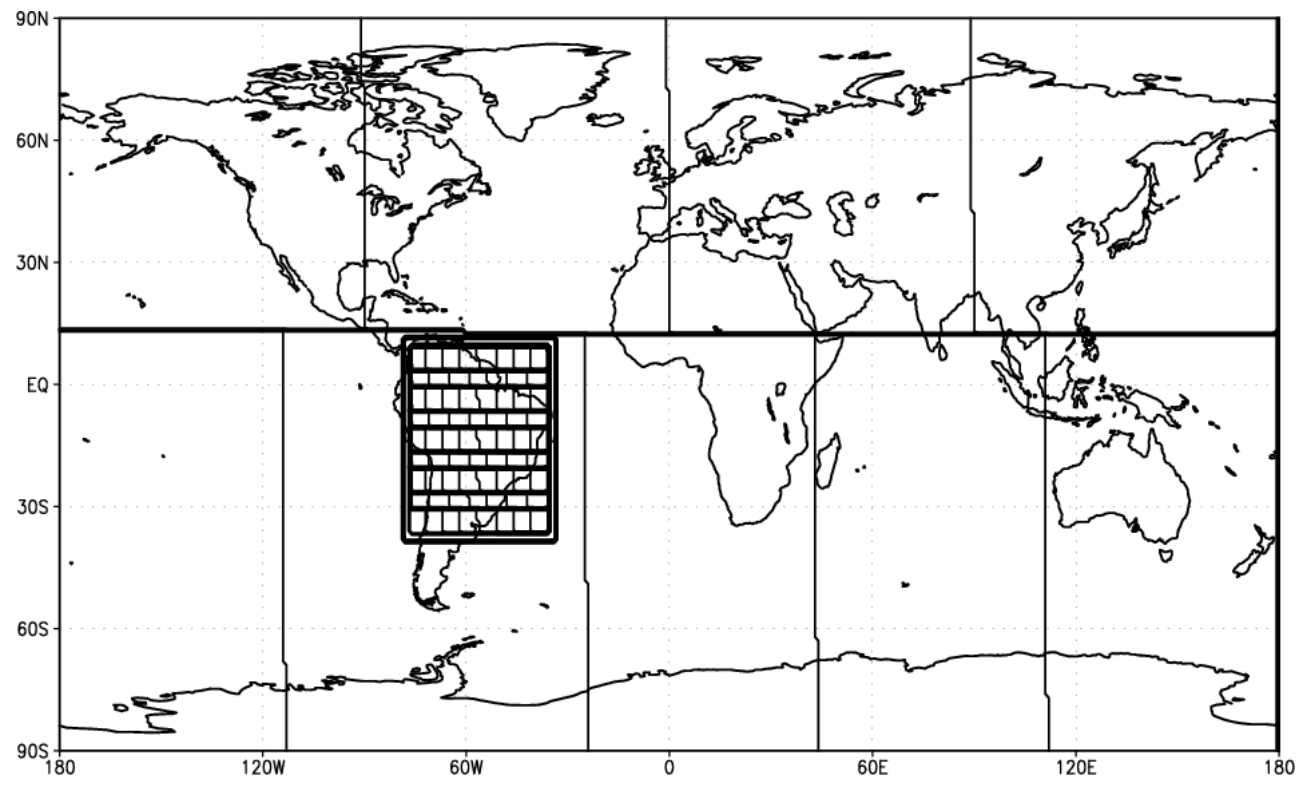

Figura 2.20: Decomposição das malhas para os dados da Tabela 2.2.

Como último exemplo, a Figura 2.21 mostra a partição obtida para a malha grossa, respectiva aos dados da Tabela 2.3, que correspondem a uma divisão entre 237 processadores. Destes exemplos observamos uma diminuição no desbalanço no número de pontos por processador conforme o número de processadores aumenta. 


\begin{tabular}{|c|c|c|c|c|c|}
\hline região & $\mathrm{npts}$ & $\%$ & $\mathrm{np} / \mathrm{reg}$ & $\mathrm{npts} / \mathrm{pr}$ & $\%$ des \\
\hline \hline grossa & 62910 & 11.52 & 28 & 2247 & -2.52 \\
\hline trans & 7428 & 1.36 & 4 & 1866 & -19.05 \\
\hline fina & 475881 & 87.12 & 205 & 2322 & 0.73 \\
\hline \hline (ideal) & 546219 & 100.00 & 237 & 2305 & - \\
\hline
\end{tabular}

Tabela 2.3: Pontos por processador para cada região e para o caso ideal, para 237 processadores.

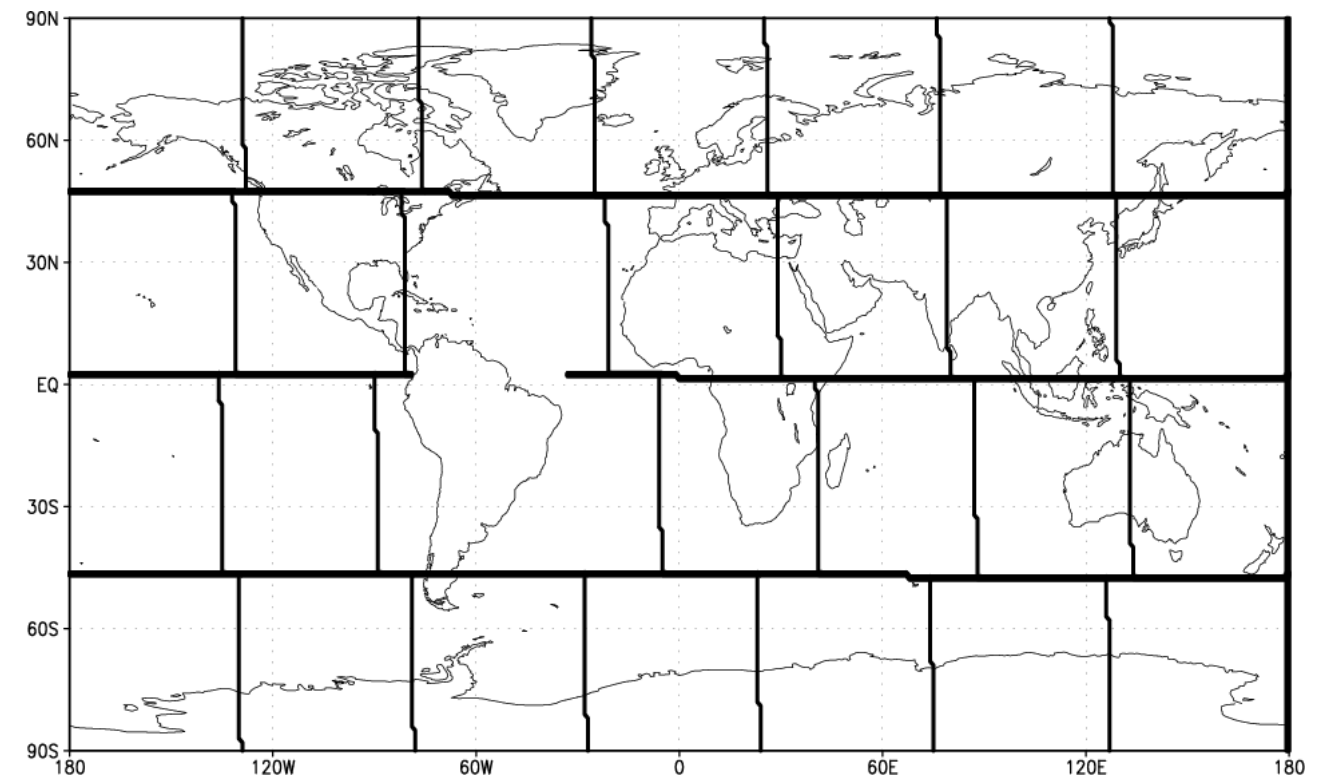

Figura 2.21: Decomposição da malha grossa para os dados da Tabela 2.3.

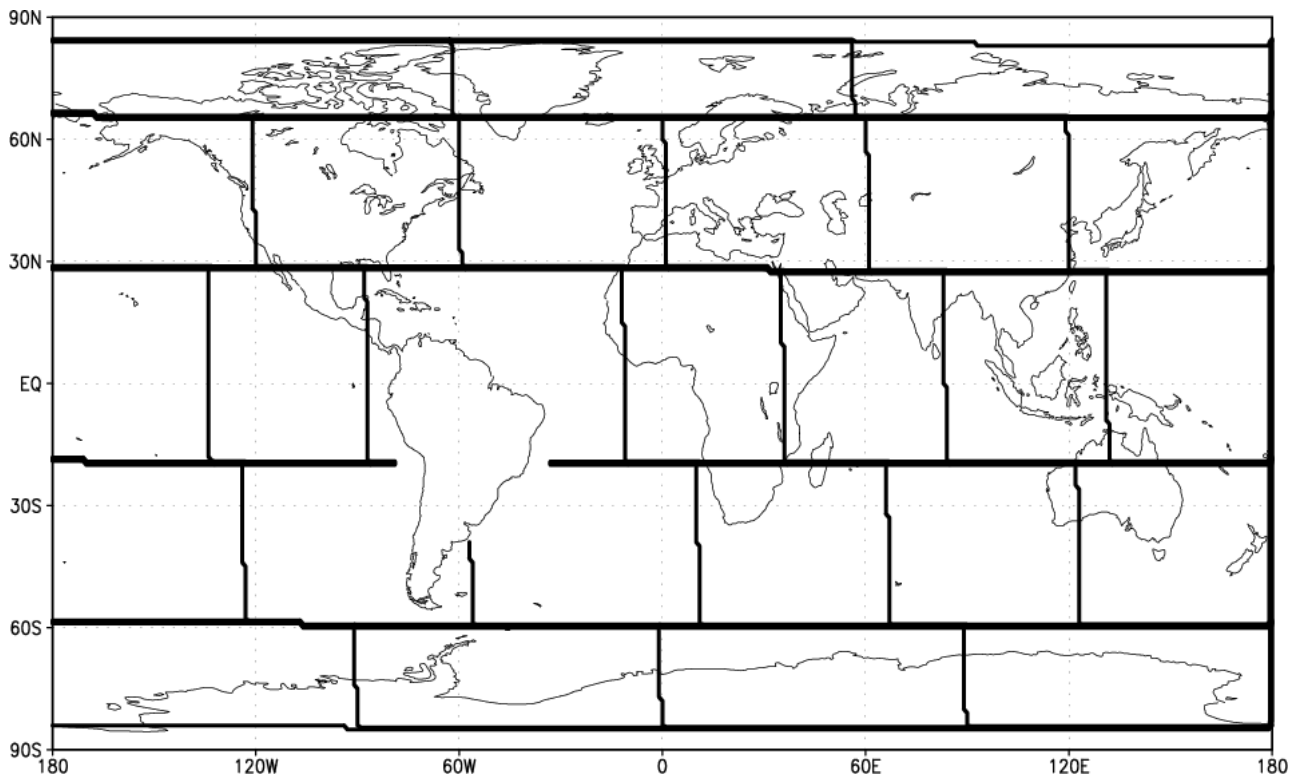

Figura 2.22: Decomposição da malha grossa para os dados da Tabela 2.3, utilizando a técnica IGLOO. 


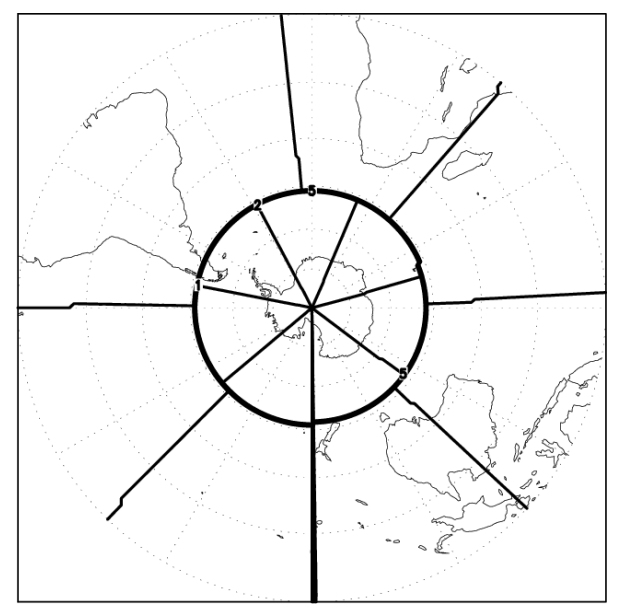

Figura 2.23: Projeção estereográfica da malha grossa, para a Tabela 2.3.

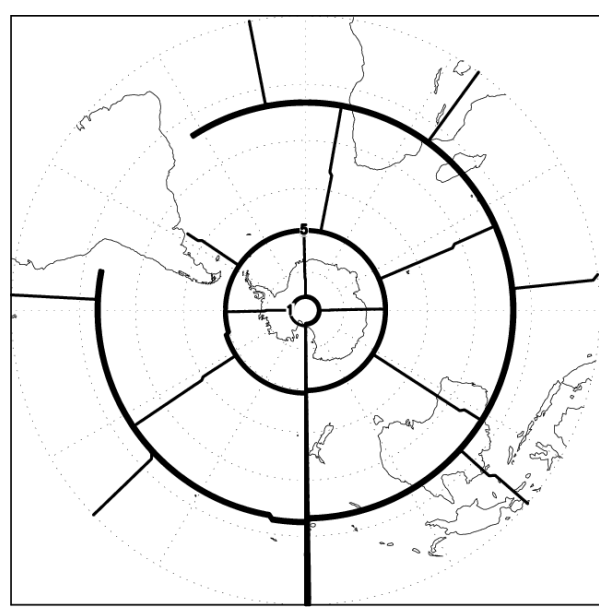

Figura 2.24: Projeção estereográfica da malha grossa para a Tabela 2.3, utilizando a técnica IGLOO. 


\subsection{Pontos inativos e comunicação}

O processo de decomposição de malhas apresentado anteriormente é para os pontos ativos, isto é, pontos de malha (F, U ou V) onde é calculada a solução das equações discretas do modelo. No entanto, faz-se necessária a utilização de pontos inativos, ou pontos onde não é calculada a solução das equações e que servem para armazenar valores, interpolados ou não, de pontos de malhas em níveis mais grossos/finos ou de pontos que estão alocados em outros processadores e que são necessários para o processamento dos pontos ativos do remendo alocados no processador. Distinguimos três tipos de pontos inativo:

1. Pontos do tipo ghost ou fantasmas: são pontos inativos que permitem o cálculo dos operadores discretos das equações (gradiente, divergente, etc.) e são determinados a partir das seguintes regras:

- Se $F_{i, j}$ é um ponto ativo, então precisamos dos pontos $F_{i, j-1}, F_{i-1, j}, F_{i+1, j}$ e $F_{i, j+1}$.

- Se $F_{i, j}$ é um ponto ativo, então precisamos dos pontos $U_{i-\frac{1}{2}, j}$ e $U_{i+\frac{1}{2}, j}$.

- Se $F_{i, j}$ é um ponto ativo, então precisamos dos pontos $V_{i, j-\frac{1}{2}}$ e $V_{i, j+\frac{1}{2}}$.

- Se $U_{i+\frac{1}{2}, j}$ é um ponto ativo, então precisamos dos pontos $V_{i, j-\frac{1}{2}}, V_{i+1, j-\frac{1}{2}}, V_{i, j+\frac{1}{2}} \mathrm{e}$ $V_{i+1, j+\frac{1}{2}}$.

- Se $V_{i, j+\frac{1}{2}}$ é um ponto ativo, então precisamos dos pontos $U_{i-\frac{1}{2}, j}, U_{i+\frac{1}{2}, j}, U_{i-\frac{1}{2}, j+1}$ e $U_{i+\frac{1}{2}, j+1}$.

Se algum destes pontos ghost estiver localizado em uma malha mais grossa ou mais fina, então são calculadas as suas dependências ou pontos desta malha grossa/fina que garantam a obtenção do valor do ponto por interpolação. Utilizamos interpolação cúbica para a malha $\mathrm{F}$ e interpolação linear para as malhas $\mathrm{U}$ e $\mathrm{V}$.

2. Pontos do tipo halo: são todos aqueles pontos inativos, normalmente alocados em processadores vizinhos, necessários para efetuar o transporte Semi-Lagrangiano, isto é, o cálculo dos pontos de partida dos pontos ativos e a interpolação (quase cúbica) das tendências das equações nos mesmos (veja a equação (1.18). A extensão do halo depende do tamanho do passo do tempo e do provável vento máximo.

3. Pontos do tipo sldep: são os pontos inativos que permitem o cálculo dos pontos de partida das malhas U e V a partir dos pontos de partida da malha F. Seja por exemplo o ponto $U_{i+\frac{1}{2}, j}$ da malha $\mathrm{U}$, seu correspondente ponto de partida é calculado como a média dos pontos de partida dos dois pontos da malha $\mathrm{F}$ mais próximos, isto é, $F_{i, j} \mathrm{e}$ $F_{i+1, j}$. De forma análoga, o ponto de partida para o ponto $V_{i, j+\frac{1}{2}}$ é obtido a partir da média dos pontos de partida dos dois pontos mais próximos da malha $\mathrm{F}$, isto é, de $F_{i, j}$ e $F_{i, j+1}$. Quando o valor para este tipo de ponto não estiver definido, ele é calculado utilizando extrapolação linear. 
Para cada um destes três tipos de pontos é realizado o seguinte procedimento: uma vez determinado o conjunto de pontos e as dependências que um processador necessita receber, este conjunto é dividido uma única vez durante uma etapa de pré-processamento, com o auxílio do mapa, segundo os processadores de procedência, e uma lista de vizinhos é criada, assim como um descritor de pontos por vizinho. Em seguida ocorre uma comunicação de descritores em duas etapas. Na primeira delas, cada processador envia aos outros uma mensagem contendo apenas o número de segmentos por nível de malha que necessita desse processador. Desta forma, cada processador recebe dos outros um vetor com números maiores ou iguais a zero. Se todos os números deste vetor forem iguais a zero, então o processador que enviou a mensagem e o que a recebeu não são vizinhos, concluindo assim o processo de comunicação. Caso contrário os processadores de origem e de destino são vizinhos e ocorre a segunda etapa, onde o processador de destino recebe do processador de origem a lista de segmentos por nível de malha em que o primeiro necessita deste último. Finalmente o processador de destino cria uma lista de vizinhos para os quais necessita enviar dados junto a um descritor de pontos por vizinho.

Durante a integração do modelo, a cada iteração e antes de serem utilizados, cada processador envia os dados que seus vizinhos necessitam e por sua vez recebe dos seus vizinhos os dados que ele necessita. Esta passagem de mensagens é realizada utilizando a biblioteca MPI (Message Passing Interface) [5].

\subsection{Paralelização do Multigrid}

Como mencionado no Capítulo 1, a equação (1.56) representa um sistema de $N_{\sigma}$ equações bidimensionais e independentes do tipo Helmholtz que resulta do desacoplamento vertical da equação tridimensional elíptica (1.55). A resolução desta equação (neste caso, do sistema de equações) é uma parte essencial de um modelo de previsão do tempo. Uma vez que estas equações são independentes, podemos explorar essa independência atribuindo um número igual de processadores a cada equação bidimensional e resolvê-las de forma independente. Ainda mais, como de modo geral a melhor eficiência de um método multigrid paralelo é obtida quando o problema é resolvido por um número de processadores igual a uma potência de dois, decidimos impor uma segunda restrição: o número total de processadores precisa ser o número de camadas verticais multiplicado por uma potência de dois.

Em [16] é mencionado que cada equação de (1.56), discretizada por diferenças finitas em uma malha uniforme longitude-latitude, conduz a um operador altamente anisotrópico na direção longitudinal próximo dos polos. Portanto, para termos um método multigrid eficiente, necessitamos utilizar um método de suavização por linhas [50], em que as variáveis para uma mesma latitude sejam atualizadas simultaneamente por meio da solução de um sistema tridiagonal em cada latitude (sistema tridiagonal periódico em caso de estarmos na malha base). Por estes motivos, decidimos mudar o sentido de paralelização, única e 
exclusivamente para resolver o sistema de equações bidimensionais (1.56), decompondo o domínio inicialmente na direção vertical e em seguida na direção latitudinal, mantendo intacta a direção longitudinal.

\subsubsection{Decomposição vertical}

Como o número de processadores dividido pelo número de camadas verticais é um número inteiro (potência de dois), digamos $p$, então criamos $N_{\sigma}$ grupos de $p$ processadores cada e o associamos a um intra-comunicador. Desta forma, a comunicação dentro de cada grupo é realizada de forma independente.

\subsubsection{Decomposição horizontal}

Uma vez feita a decomposição vertical podemos nos dedicar à paralelização de uma camada vertical, pois todas elas serão paralelizadas de forma idêntica. A seguir lembramos os principais componentes do método multigrid escolhido:

1. Formulação FAS (Full Approximation Scheme), compatível com o uso de malhas localmente refinadas.

2. Engrossamento de malhas padrão (standard coarsening), isto é, definida a malha base e os refinamentos locais, cada malha global grossa é definida dobrando sucessivamente o espaçamento horizontal da malha mais fina.

3. Suavização por linhas do tipo Zebra.

4. Restrição full-weighting padrão. Observamos que em [13] é desenvolvido um operador de restrição full-weighting transformado, que leva em consideração a geometria da esfera, mas que junto à suavização Zebra por linhas torna-se o operador full-weighting padrão. Nos polos (pontos singulares) utilizamos a definição especial do operador desenvolvida em [13]. Adicionalmente, devido à formulação FAS, utilizamos também o operador de injeção.

5. Interpolação bilinear.

6. Ciclo V(1,1) e 10 suavizações na malha mais grossa.

7. Modo FMG (full-multigrid), que nos permite obter uma aproximação inicial próxima da solução.

Embora os componentes do método multigrid sejam altamente paralelizáveis, o método como um todo não é, principalmente por dois motivos. Primeiro, as malhas precisam ser processadas sequencialmente e, segundo, o grau de paralelismo é diferente em cada nível (pequeno em malhas grossas). 
Inicialmente paralelizamos as malhas globais no sentido latitudinal. Dividimos o número de latitudes da malha global mais fina entre o número de processadores, impondo que cada processador tenha como mínimo $q_{0}$ latitudes para processar (neste trabalho, $q_{0}=4$ ). A divisão das malhas globais mais grossas é feita de modo similar, mas como o número de latitudes diminui conforme a malha torna-se mais grossa, avaliamos se cada processador possui como mínimo $q_{0}$ latitudes para processar. Se esse for o caso, a partição deste nível permanece da mesma forma que o nível global imediatamente superior. Caso contrário nos encontramos na situação em que os processadores possuem um número pequeno de latitudes para processar, levando a um aumento no custo de comunicação. Neste caso realizamos um processo de aglomeração [52], que consiste em agrupar as linhas em novas unidades de processo e em redistribuir estas entre um subconjunto de processadores, reduzindo desta forma a comunicação. Como exemplo, suponhamos que temos uma malha com 16 latitudes e 8 processadores, numerados de 0 a 7 . Desta forma, cada um deles teria $2<q_{0}$ latitudes para processar. Em vez disso, juntamos as primeiras quatro latitudes e as entregamos para serem processadas por ambos os processadores 0 e 1 . De forma análoga os processadores 2 e 3 processarão as 4 seguintes latitudes, os processadores 5 e 6 as seguintes 4 e finalmente os processadores 6 e 7 as últimas quatro latitudes. Embora possamos observar uma replicação do trabalho (os processadores 1, 3,5 e 7 fazem o mesmo trabalho que os processadores 0 , 2, 4 e 6, respectivamente), este trabalho é pequeno, pois estamos em uma malha grossa, comparado com custo de comunicação reduzido.

Uma vez divididas as malhas globais, dividimos as malhas locais, assumindo que para estas não será necessário o uso da técnica de aglomeração. O processo de paralelização ocorre de forma similar ao das malhas globais mas com uma diferença: implementamos um processo heurístico para reordenar os processadores de forma que, quando for possível, a restrição das suas respectivas latitudes sobre a malha global fiquem dentro das suas respectivas latitudes nestas últimas. Observamos que este reordenamento depende da posição dos refinamentos locais na malha base.

\section{Suavização Zebra}

O método de suavização por linhas do tipo Zebra consta de duas etapas. Na primeira, atualizamos as latitudes pares, para em seguida atualizar as latitudes impares. Estas atualizações resultam da resolução de um sistema tridiagonal (periódico em caso da malha ser global). Cada uma destas etapas ocorre da mesma forma que em uma versão sequencial, com a diferença que na versão paralela cada processador trabalha sobre seu conjunto de latitudes e que antes de realizar cada uma das etapas precisamos determinar se é necessário enviar/receber dados aos/dos processadores vizinhos ao norte e ao sul para poder montar os sistemas tridiagonais mencionados. 


\section{Operadores de restrição}

Transferimos os resíduos de uma malha fina para uma malha grossa utilizando o operador full-weighting, e a solução utilizando o operador injeção. Antes de calcular o resíduo nas linhas pares, pois nas ímpares o valor é zero devido à suavização Zebra, devemos verificar se necessitamos enviar/receber dados aos/dos processadores ao norte e ao sul. Uma vez realizado isto, a restrição do resíduo ocorre da mesma forma que em um programa sequencial. Já para a injeção da solução, não é necessária comunicação alguma.

\section{Operador de interpolação}

Transferimos as correções de uma malha grossa para uma malha fina utilizando interpolação bilinear. Mas antes de efetuar isto necessitamos determinar se é necessário enviar/receber dados de correções aos/dos processadores vizinhos ao norte e ao sul. Uma vez feito isto, a interpolação ocorre da mesma forma que em um programa sequencial. Se a interpolação é feita sobre uma malha local, então utilizamos interpolação cúbica no cálculo das correções na fronteira da mesma.

\section{Formulação FAS}

Quando utilizamos a formulação FAS, trabalhamos com a solução na malha grossa em vez de trabalhar com o erro, e necessitamos, após a restrição do resíduo e da solução, atualizar o lado direito da equação. Antes de realizar esta atualização, necessitamos determinar se é necessário enviar/receber a solução, na malha grossa, aos/dos processadores vizinhos ao norte e ao sul. Uma vez feito isto, a atualização ocorre da mesma forma que na versão sequencial.

\section{Modo FMG}

Utilizamos o modo FMG (full-multigrid) para obter aproximações iniciais em malhas finas a partir da interpolação (cúbica) da solução em malhas grossas. Antes de efetuar a interpolação devemos verificar se necessitamos enviar/receber a solução aos/dos processadores vizinhos ao norte e ao sul. Uma vez realizado isto a interpolação ocorre da mesma forma que em um programa sequencial.

\subsection{Transposição dos dados}

Como mencionamos anteriormente, mudamos o sentido do paralelismo única e exclusivamente para resolver o sistema de equações bidimensionais do tipo Helmholtz (1.56). Isto significa que uma vez calculado o lado direito de (1.56), necessitamos reordenar estes dados de forma que possam ser utilizados pelo método multigrid e, uma vez calculadas as soluções 
do sistema (1.56), necessitamos reordenar novamente estes dados de forma em que o modelo possa utilizá-los para calcular os novos valores dos campos e continuar com a seguinte iteração da integração. Realizamos isto aplicando a técnica de transposição de dados, desenvolvida em [14], que consiste em uma redistribuição total dos dados entre os processos por meio de passagens de mensagens. Desta forma, durante esta etapa é comunicado um único campo: o lado direito de (1.56) ou a sua solução.

Da mesma forma que cada processador cria um descritor de malhas para seus pontos ativos durante o processo de paralelização do modelo, e que aqui chamamos de descritor da dinâmica, durante a paralelização do multigrid é criado um descritor para esta etapa, que chamaremos nesta seção de descritor multigrid. Determinamos a relação de vizinhos e pontos do lado direito de (1.56) que um processador necessita receber antes de executar o método multigrid fazendo a interseção entre o descritor multigrid desse processador e os descritores da dinâmica de todos os processadores. No caso de a interseção destes descritores ser não nula, para um mesmo processador, a mesma é separada em um descritor especial para realizar uma cópia local em vez de comunicação. Esta lista também é utilizada para enviar a solução do sistema (1.56) aos processadores vizinhos.

De forma análoga, determinamos a relação de vizinhos e pontos do lado direito de (1.56) que um processador necessita enviar, antes que seja executado o método multigrid, calculando a interseção entre o descritor da dinâmica e os descritores multigrid de todos os processadores. Novamente, caso a interseção destes descritores seja não nula para um mesmo processador, a mesma é separada em um descritor especial para efetuar uma cópia local em vez de comunicação. Esta lista também é utilizada para receber dos processadores vizinhos a solução do sistema (1.56). 


\section{Capítulo 3}

\section{Testes computacionais}

Neste capítulo apresentamos os testes computacionais que comprovam o bom desempenho de nosso modelo paralelo. Apresentamos primeiro testes do método multigrid na resolução de um problema com solução conhecida. A seguir apresentamos os testes do modelo para malhas uniformes e para malhas com refinamento local. Finalmente apresentamos os testes de desempenho de nosso modelo no cluster tupã do CPTEC $^{1}$, onde foi executado utilizando até 1024 processadores.

\subsection{Validação do método multigrid}

Iniciamos a validação do nosso modelo resolvendo uma das equações do sistema (1.56) com o lado direito compelido de forma que a solução exata seja $G(\lambda, \theta)=\sin ^{2}(\lambda) \cos ^{3}(\theta)$ [13]. Utilizamos o valor $\tau=3600 \mathrm{~s}$ e os valores dos três maiores autovalores da matriz $\mathbf{E}$ (para os menores a convergência é ainda mais rápida) obtida por meio de uma discretização vertical uniforme de 26 níveis. A tabela abaixo mostra a norma do máximo do erro e os fatores de convergência, entre parênteses, obtidos após a execução de um ciclo de um método multigrid com suavização Zebra, ciclo V(1,1), esquema FAS e modo FMG para malhas uniformes em diferentes resoluções. Os resultados evidenciam uma ordem de convergência igual a 2.

\begin{tabular}{|c|c|c|c|}
\hline Malha & Maior autovalor & $2^{\circ}$ maior autovalor & $3^{\circ}$ maior autovalor \\
\hline $128 \times 65$ & $3.06 \mathrm{E}-04(-)$ & $2.51 \mathrm{E}-04(-)$ & $2.39 \mathrm{E}-04(-)$ \\
\hline $256 \times 129$ & $7.61 \mathrm{E}-05(4.02)$ & $6.22 \mathrm{E}-05(4.04)$ & $5.63 \mathrm{E}-05(4.25)$ \\
\hline $512 \times 257$ & $1.90 \mathrm{E}-05(4.01)$ & $1.54 \mathrm{E}-05(4.03)$ & $1.41 \mathrm{E}-05(4.00)$ \\
\hline $1024 \times 513$ & $4.74 \mathrm{E}-06(4.00)$ & $3.86 \mathrm{E}-06(4.00)$ & $3.49 \mathrm{E}-06(4.03)$ \\
\hline
\end{tabular}

Tabela 3.1: Fatores de convergência do multigrid para a equação (1.56) e os três maiores autovalores

\footnotetext{
${ }^{1}$ Centro de Previsão de Tempo e Estudos Climáticos - http://www.cptec.inpe.br/
} 
Uma vez garantida a ordem de convergência 2 do método multigrid em malhas uniformes, necessitamos garanti-la para malhas com refinamentos locais. Para tal, utilizamos uma malha base com três refinamentos locais, como mostra a seguinte figura:

\begin{tabular}{|c|c|c|c|c|}
\hline Grid loca & Eions & & & \\
\hline imin_ref $=$ & 0 & 32, & 3 & \\
\hline max_ref $=$ & 127 & 96, & 125, & 24 \\
\hline jmin_ref $=$ & 0 & 16, & 3 & \\
\hline jmax_ref $=$ & 64, & 48, & 61, & \\
\hline
\end{tabular}

Figura 3.1: Configuração de malhas para o teste de convergência do método multigrid.

Utilizando esta configuração de malhas e sucessivos refinamentos das mesmas, resolvemos a equação (1.56) para o terceiro maior autovalor da matriz $\mathbf{E}$ utilizando o método multigrid do teste anterior. A Tabela 3.2 mostra a norma do máximo do erro e os fatores de convergência entre parênteses. Os resultados mostram novamente uma ordem de convergência 2.

\begin{tabular}{|c|c|}
\hline Malha base & $\|e\|_{\infty}$ \\
\hline $128 \times 65$ & $5.42 \mathrm{E}-06(-)$ \\
\hline $256 \times 129$ & $1.37 \mathrm{E}-06(3.95)$ \\
\hline $512 \times 257$ & $3.41 \mathrm{E}-07(4.02)$ \\
\hline $1024 \times 513$ & $8.51 \mathrm{E}-08(4.01)$ \\
\hline
\end{tabular}

Tabela 3.2: Fatores de convergência do multigrid para a equação (1.56) em malhas localmente refinadas.

\subsection{Validação do modelo}

A validação de modelos que resolvem as equações primitivas é uma etapa importante no desenvolvimento de modelos de circulação geral da atmosfera (GCMs), pois revela a influência do desenho do modelo nas simulações do tempo e clima e indica se as variáveis do modelo são descritas com precisão pela aproximação numérica. Uma das principais dificultades da validação é a ausência de uma solução analítica conhecida. Em [39] é proposto um teste que consiste na integração, por um periodo de 12 dias, das equações primitivas não forçadas com condição inicial prescrita por um jato de latitudes médias baroclínicamente instável e especificado de forma analítica. Entretanto, este teste necessita a inclusão explícita de um termo difusivo no lado direito das equações da quantidade de movimento e da termodinâmica para dissipar processos de escala sub-malha e prevenir singularidades em um tempo finito.

Un outro teste, proposto em [33] e [32], consiste na evolução de uma onda baroclínica idealizada no hemisfério norte por um periodo de dez dias, com condição inicial descrita 
analíticamente junto a uma perturbação local não periódica. Esta condição inicial é uma solução analítica das equações primitivas hidrostáticas e em uma primeira etapa, quando utilizada sem a perturbação, permite avaliar a capacidade do modelo em manter dita solução ao longo de tempo antes de que os erros de truncamento e arredondamento, assim como as ondas de gravidade, degradem o estado em regime permanente. Já em uma segunda etapa é introduzida uma perturbação no vento zonal, em latitudes médias, que desencadeia o crescimento de uma onda baroclínica no hemisfério norte ao longo de vários dias, para a qual não é conhecida uma solução analítica.

Validamos o nosso modelo utilizando o teste proposto em [33] e [32], com os campos e parâmetros definidos em [33] e uma discretização vertical uniforme em coordenadas $\sigma=$ $p / p_{s}$ com 26 níveis. Adicionalmente, como nosso modelo utiliza uma discretização de três níveis no tempo (Leapfrog), ele possui um modo computacional, cujo crescimento evitamos utilizando o filtro proposto em [54] e [55], que é uma alternativa ao filtro de Robert-Asselin [9]. Não obstante, em nossos testes preliminares, obtivemos melhores resultados utilizando o parâmetro $\alpha=1$, que recupera o filtro original. Adicionalmente utilizamos o parâmetro $\nu=0.25$.

\subsubsection{Teste em regime permanente}

Nesta primeira etapa utilizamos a condição inicial definida em [33] (sem a perturbação do vento zonal) e consideramos únicamente malhas uniformes com resoluções horizontais $128 \times 65,256 \times 129,512 \times 257$ e $1024 \times 513$. A Figura 3.2 mostra a norma $l_{2} \mathrm{em} \mathrm{m} / \mathrm{s}$, definida em [33], avaliando a perda de simetria do vento zonal em relação à sua média zonal. Observamos que o crescimento da asimetria zonal aumenta ligeiramente conforme a resolução da malha aumenta. Isto pode ser explicado pelo aumento no número de cálculo de trajetórias e de interpolações cúbicas em malhas com maior resolução.

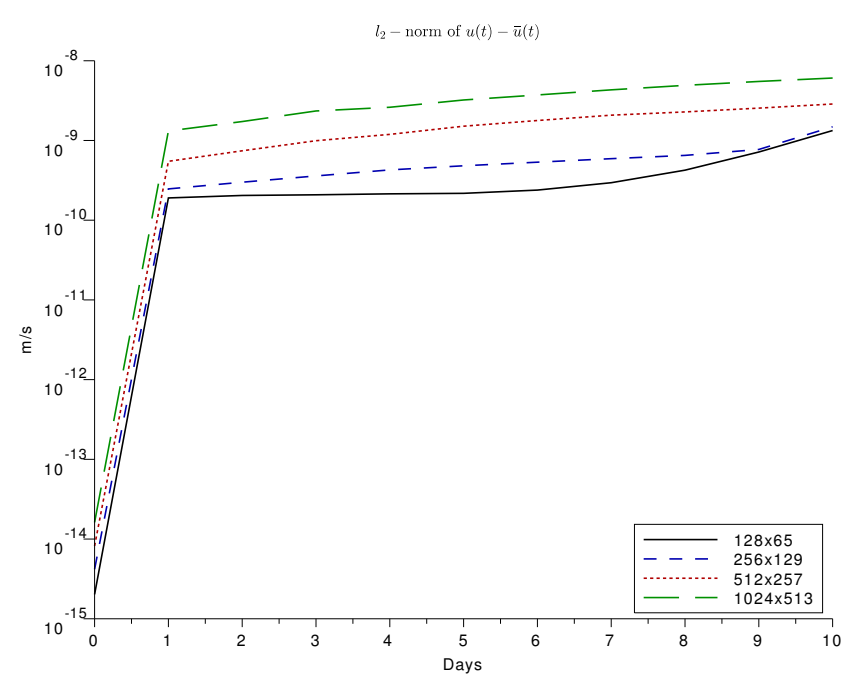

Figura 3.2: Norma $_{2}(\mathrm{~m} / \mathrm{s})$ da perda de simetria do vento zonal em relação à sua média zonal. 
Uma outra característica a ser avaliada é a degradação da média zonal do vento zonal em relação à solução exata, que mostramos na Figura 3.3. Observamos que esta degradação é pequena, aumenta pouco com o tempo e que praticamente não ha melhoras com o aumento da resolução horizontal.

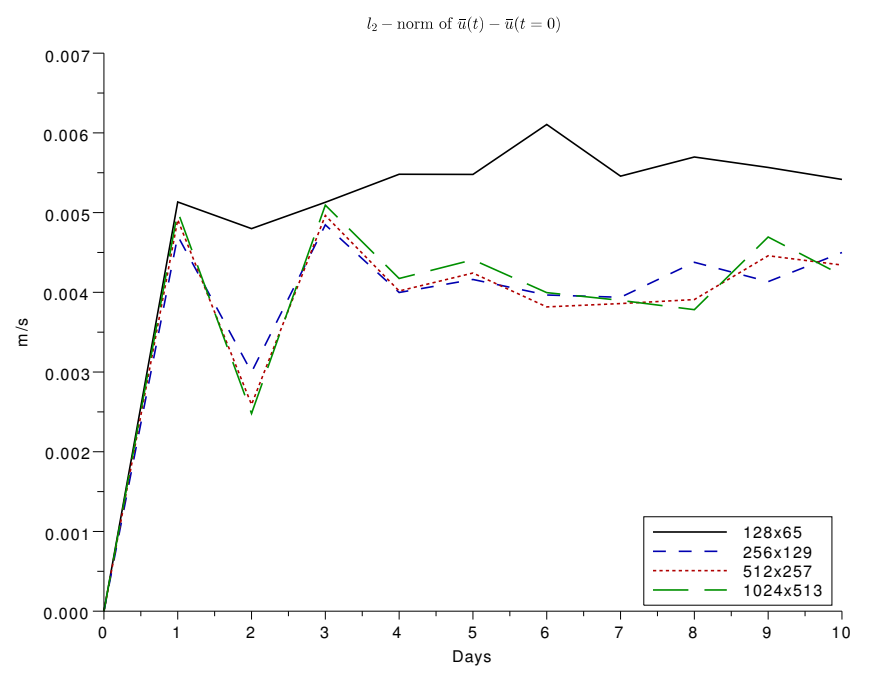

Figura 3.3: Norma $_{2}(\mathrm{~m} / \mathrm{s})$ da degradação da média zonal do vento zonal em relação à solução exata.

\subsubsection{Teste da onda baroclínica}

Nesta etapa de validação consideramos a perturbação do vento zonal junto à condição inicial, o que desencadeia o crescimento de uma onda baroclínica no hemisfério norte ao longo de vários dias. Como neste caso uma solução analítica é desconhecida, determinamos uma solução numérica de referência, utilizando uma malha com resolução horizontal de $1024 \times 513$ pontos, 26 níveis na vertical e passo de tempo igual a 450s. A Figura 3.4 mostra a evolução temporal da pressão de superfície e da temperatura em $850 \mathrm{hPa}^{2}$ para o hemisfério norte nos dias 4, 6, 7, 8, 9 e 10. Observamos que a onda baroclínica cresce muito lentamente até o dia 4. No dia 6 a pressão de superfície mostra dois sistemas de alta e baixa pressão (Figura 3.4c) enquanto a temperatura exibe duas ondas de pequena amplitude (Figura 3.4d). No dia 7 o estágio de rápido desenvolvimento da onda baroclínica é iniciado (note a mudança de escala). No dia 8 os centros das altas e baixas de pressão têm se acentuado significativamente (Figura $3.4 \mathrm{~g}$ ) e as ondas no campo da temperatura, onde se observa uma terceira à esquerda das anteriores, quase têm atingido seu ponto máximo e começam envolver em volta gerando frentes a partir delas. No dia 9 os centros das altas e baixas de pressão ficam ainda mais acentuadas (Figura 3.4i) assim como as ondas da temperatura (Figura 3.4j). No dia 10 ocorre a quebra da onda. Três células fechadas com temperaturas ligeiramente maiores têm

\footnotetext{
${ }^{2}$ A temperatura em $850 \mathrm{hPa}$ foi obtida por meio de uma interpolação linear vertical em coordenadas $\ln p$.
} 
se formado precedidas por uma frente muito acentuada. Observamos que os nossos resultados estão de acordo com os relatados em [33].

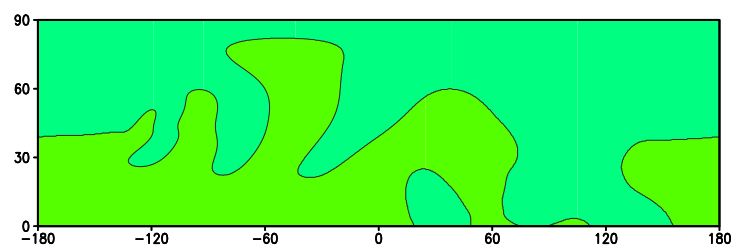

(a) $\operatorname{Dia} 4$

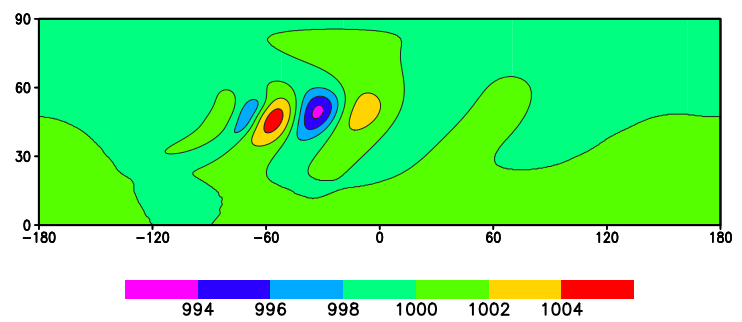

(c) Dia 6

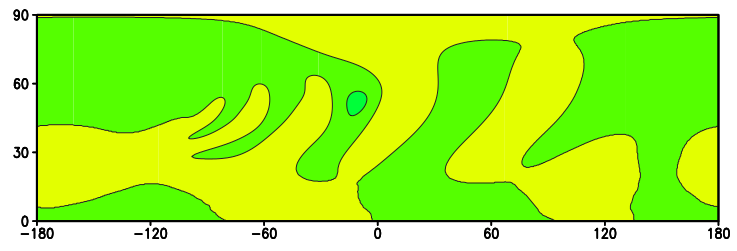

(e) Dia 7

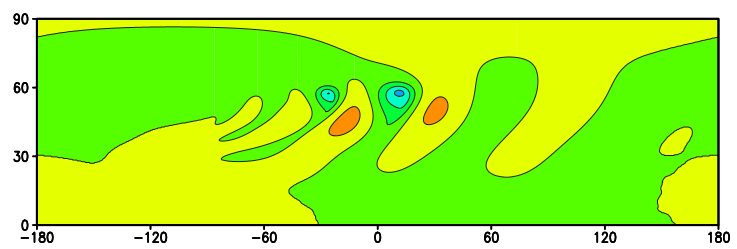

(g) Dia 8

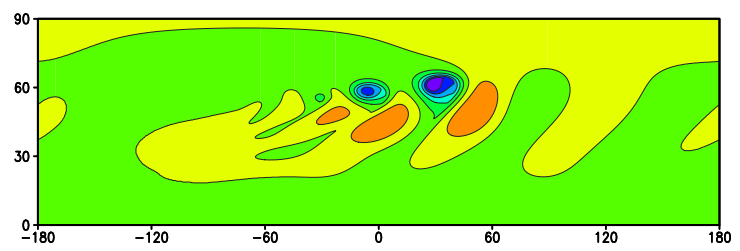

(i) Dia 9

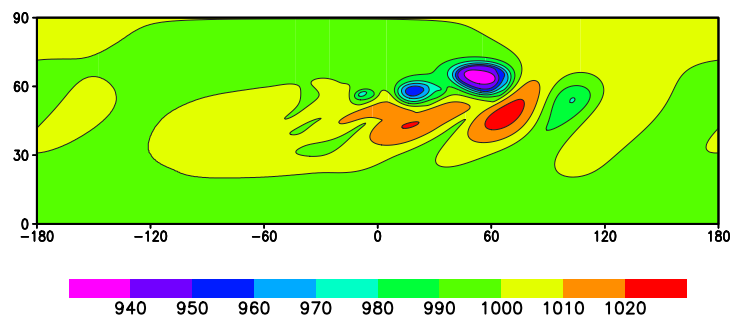

(k) Dia 10

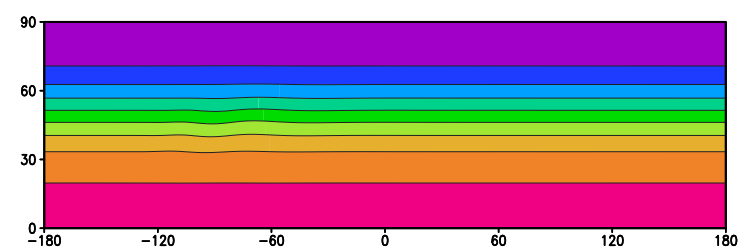

(b) $\operatorname{Dia} 4$

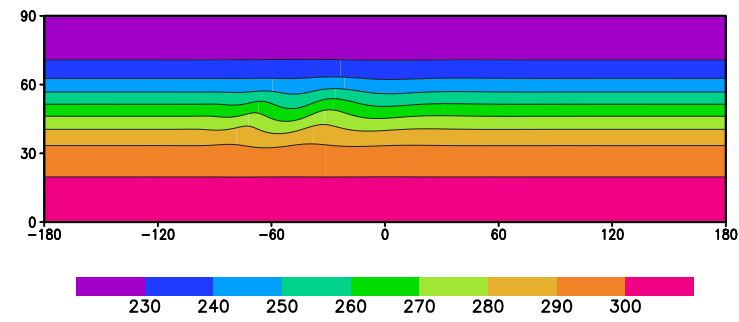

(d) Dia 6

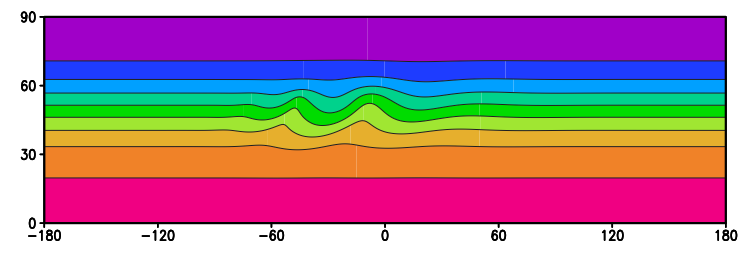

(f) Dia 7

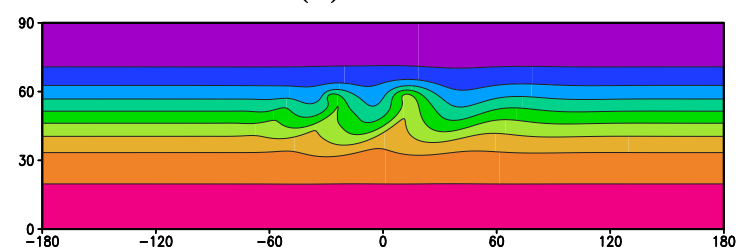

(h) Dia 8

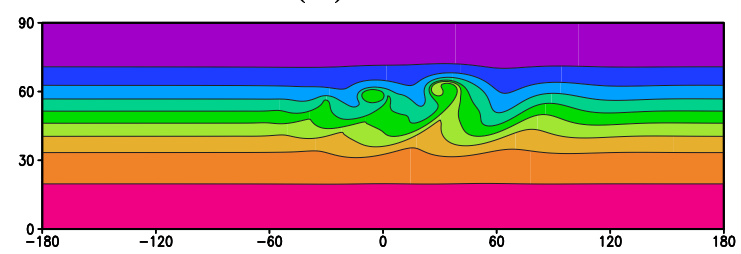

(j) Dia 9

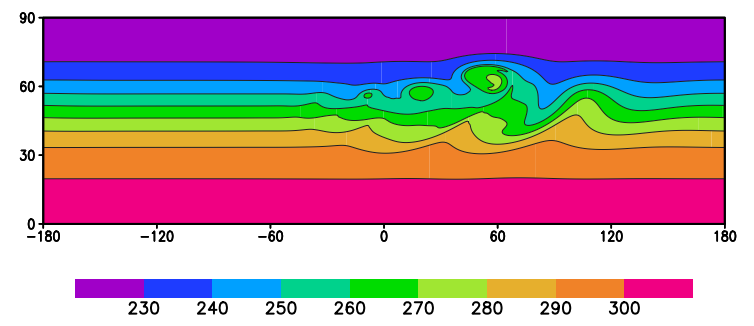

(1) Dia 10

Figura 3.4: Evolução da pressão de superfície (esquerda) e da temperatura em 850hPa (direita) para uma malha com resolução horizontal de $1024 \times 513$ pontos, 26 niveis verticais e $\Delta t=450$ s. 
Apresentamos a seguir os resultados obtidos com diferentes resoluções horizontais, os quais evidenciam uma convergência numérica das soluções conforme a resolução da malha aumenta. A Figura 3.5 mostra a pressão de superfície e a temperatura em $850 \mathrm{hPa}$ para o hemisfério norte após 9 dias, para as resoluções horizontais, de cima para baixo, $128 \times 65$, $256 \times 129,512 \times 257$ e $1024 \times 513$. Os tamanhos do passo de tempo utilizados foram 3600 s. 1800s, 900s e 450s, respectivamente. Em todos os casos utilizamos 26 níveis verticais. Observamos que para as duas resoluções mais baixas os resultados não capturam a intensidade das células fechadas na pressão de superfície. No entanto, os resultados das duas malhas mais finas são muito semelhantes.

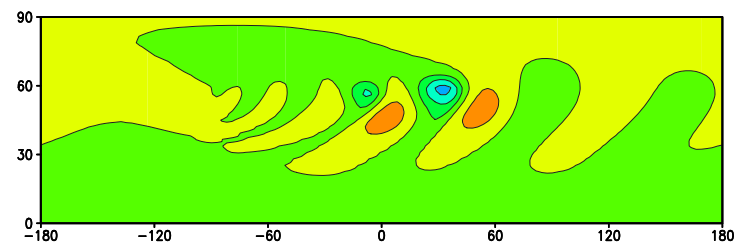

(a) Malha $128 \times 65$

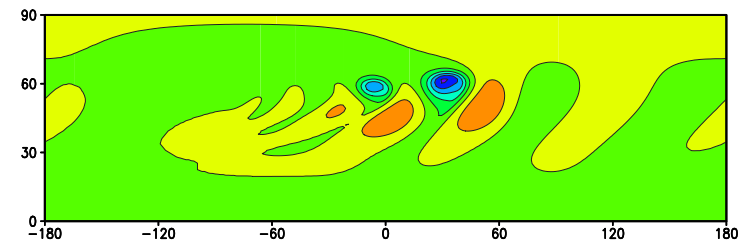

(c) Malha $256 \times 129$

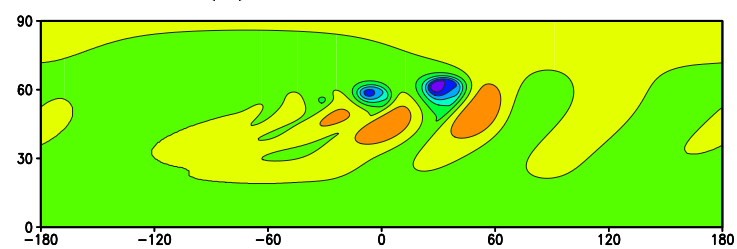

(e) Malha $512 \times 257$

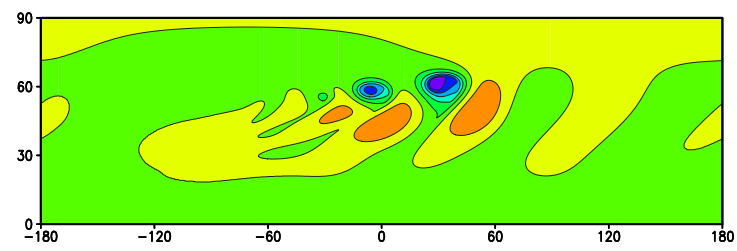

(g) Malha $1024 \times 513$

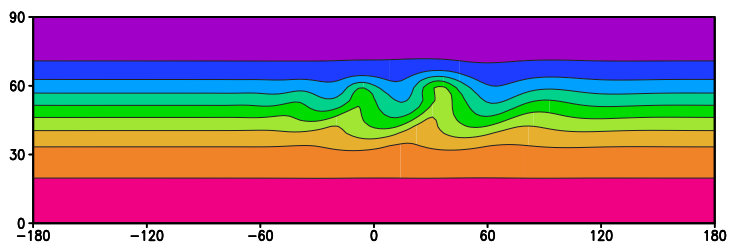

(b) Malha $128 \times 65$

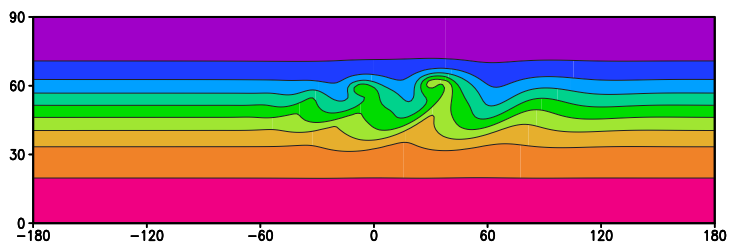

(d) Malha $256 \times 129$

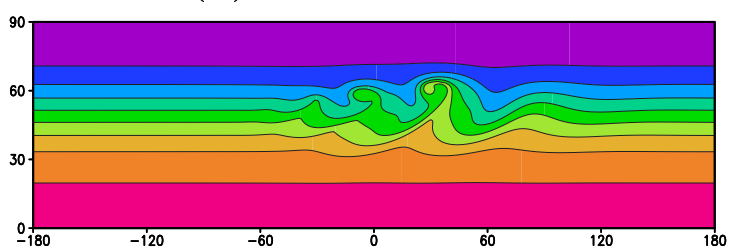

(f) Malha $512 \times 257$

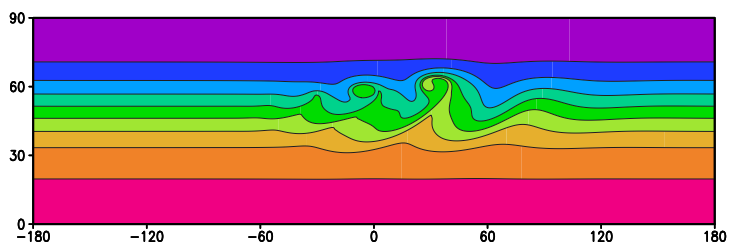

(h) Malha $1024 \times 513$

Figura 3.5: Pressão de superfície (esquerda) e temperatura em 850hPa (direita) após 9 dias.

De forma análoga, utilizando a mesma configuração, a Figura 3.6 mostra a pressão de superfície e a temperatura em $850 \mathrm{hPa}$ após 10 dias. Novamente observamos que para as duas resoluções mais baixas os resultados não capturam a intensidade das células fechadas na pressão de superfície ao passo que para as duas resoluções mais finas os resultados são muito semelhantes.

Para quantificar a ordem de convergência do modelo as Figuras 3.7 e 3.8 mostram a norma $l_{2}$ do erro na pressão de superfície e na temperatura para $\sigma=0.865$ respectivamente, em relação à solução de referência. Ambas evidenciam uma ordem de convergência 2. 


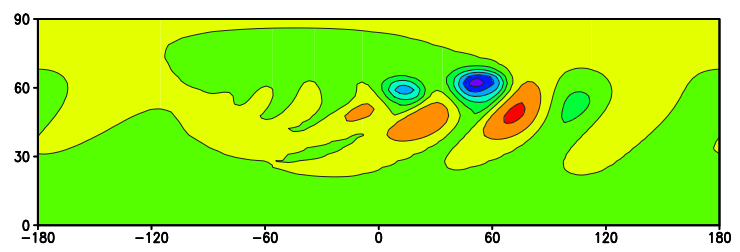

(a) Malha $128 \times 65$

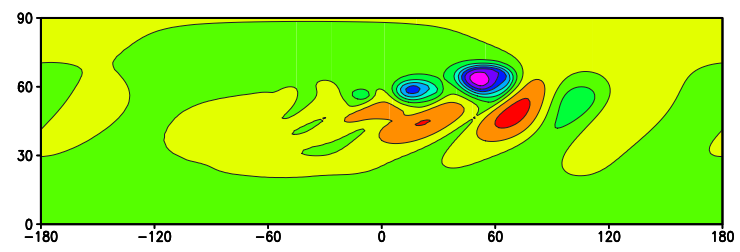

(c) Malha $256 \times 129$

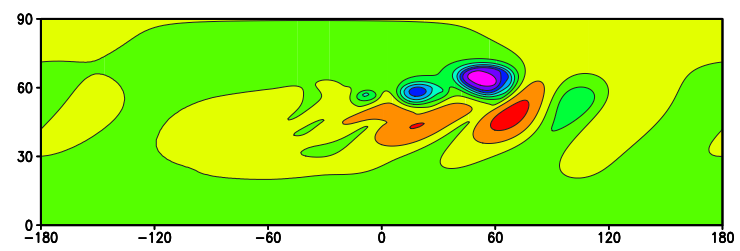

(e) Malha $512 \times 257$

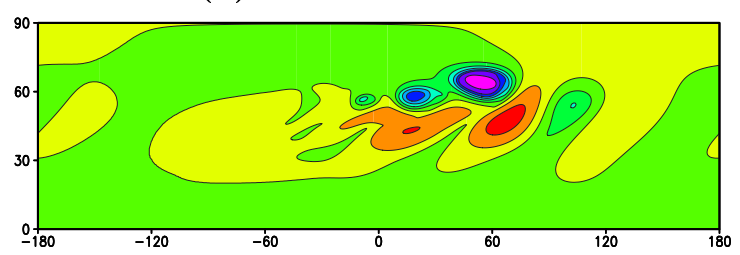

(g) Malha $1024 \times 513$

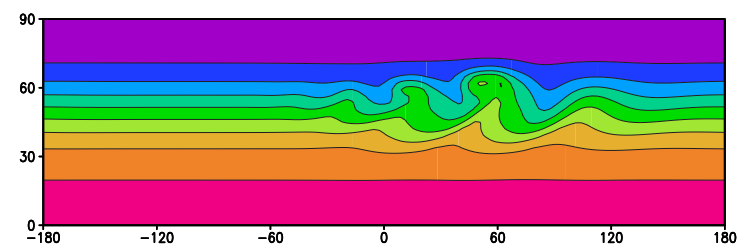

(b) Malha $128 \times 65$

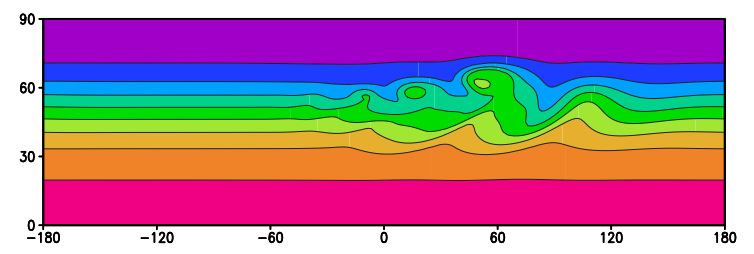

(d) Malha $256 \times 129$

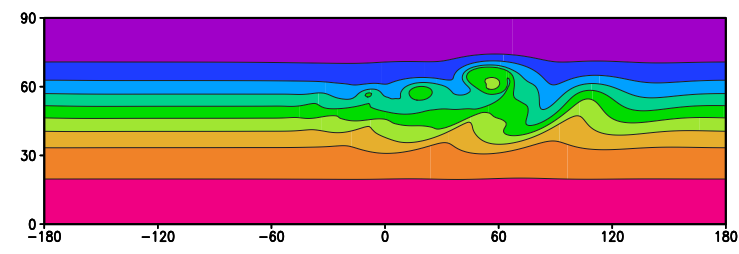

(f) Malha $512 \times 257$

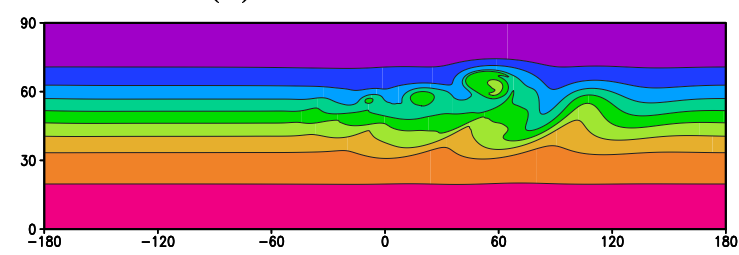

(h) Malha $1024 \times 513$

Figura 3.6: Pressão de superfície (esquerda) e temperatura em 850hPa (direita) após 10 dias.

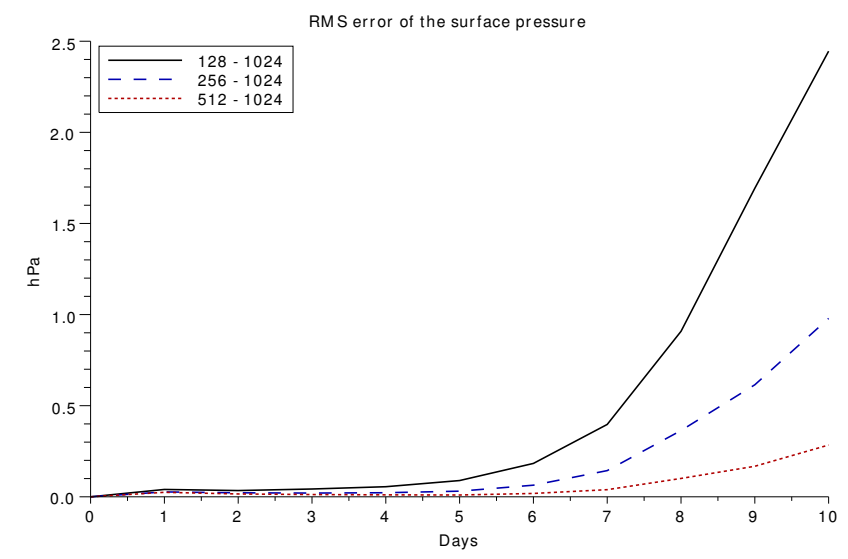

Figura 3.7: Norma $_{2}$ (hPa) da variação da pressão de superfície ao longo do tempo para varias resoluções, em relação à resolução de referência $1024 \times 513 \times 26$.

\subsubsection{Teste da onda baroclínica com refinamentos locais}

Uma vez validado o nosso modelo, em malhas uniformes, com o teste proposto em [33], procedemos a valida-lo utilizando refinamentos locais. Para efetuar isto consideramos a nossa solução de referência e um conjunto de malhas formado por uma malha grossa global e três 


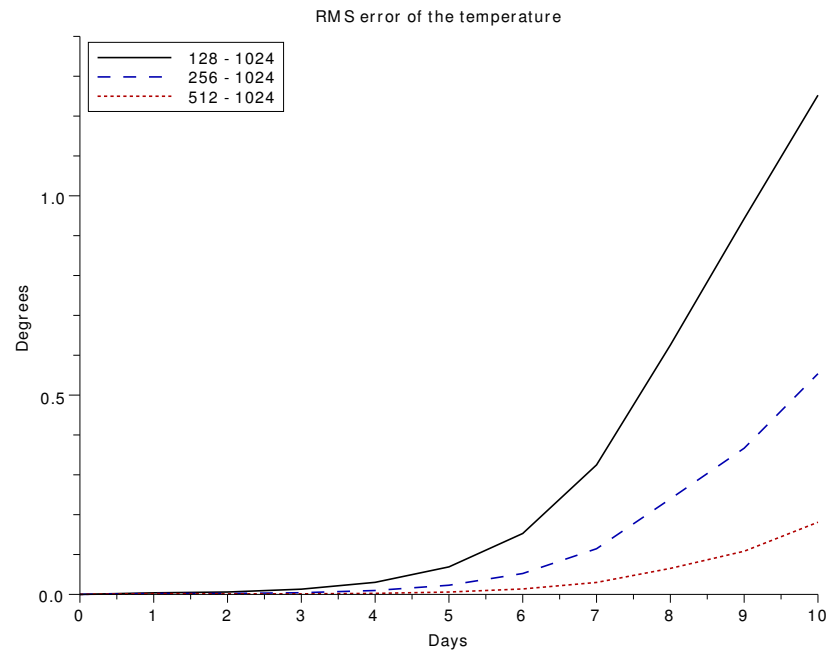

Figura 3.8: Norma $_{2}\left({ }^{\circ} \mathrm{K}\right)$ da variação da temperatura, no nível $\sigma=0.865$, ao longo do tempo para varias resoluções, em relação à resolução de referência $1024 \times 513 \times 26$.

refinamentos locais, com dimensões determinadas pela configuração mostrada na seguinte figura:

! Number of refinement levels

nref $=3$

! Grid locations

imin_ref $=0,30,3,3$

imax_ref $=127,90,117,225$

jmin_ref $=0,33,3,3$

jmax_ref $=64,61, \quad 53, \quad 97$

Figura 3.9: Configuraçẫo das malhas para o teste da onda baroclínica com refinamentos locais.

Da Figura 3.9 observamos que a resolução do refinamento mais fino é igual à resolução da nossa malha de referência, pelo que fácilmente podemos comparar as soluções obtidas em ambas as malhas. Para munir o nosso conjunto de malhas de uma condição inicial tomamos os campos da nossa solução de referência, para o dia 5, e os interpolamos cúbicamente para cada uma das malhas do conjunto (para o refinamento mais fino fazemos uma cópia simples). Realizamos este procedimento por dois motivos: difícilmente o conjunto de malhas conseguirá resultados precisos em uma integração de 10 dias e, como dizemos anteriormente, o desenvolvimento da onda baroclínica acontece principalmente a partir do dia 6 . 
A Figura 3.10 mostra a localização dos refinamentos locais e a pressão de superfície inicial para as mesmas. Já a Figura 3.11 mostra a pressão de supefície (à esquerda) e a temperatura em $850 \mathrm{hPa}$ (à direita) para o refinamento mais fino (em cores) e para a malha de referência (em preto). Observamos que as altas e baixas na pressão de superfície são muito bem reproduzidas pelo refinamento local e que pequenas diferenças ocorrem em torno do valor $1000 \mathrm{hPa}$. De forma análoga, a temperatura é muito bem representada pelo refinamento local e pequenas diferenças com relação à malha de referência são observadas únicamente no dia 5. Finalizando esta etapa de validação ss figuras 3.12 e 3.13 mostram, respectivamente, a norma $l_{2}$ do erro da pressão de superfície e da temperatura em $\sigma=0.865$ em relação à solução de referência.

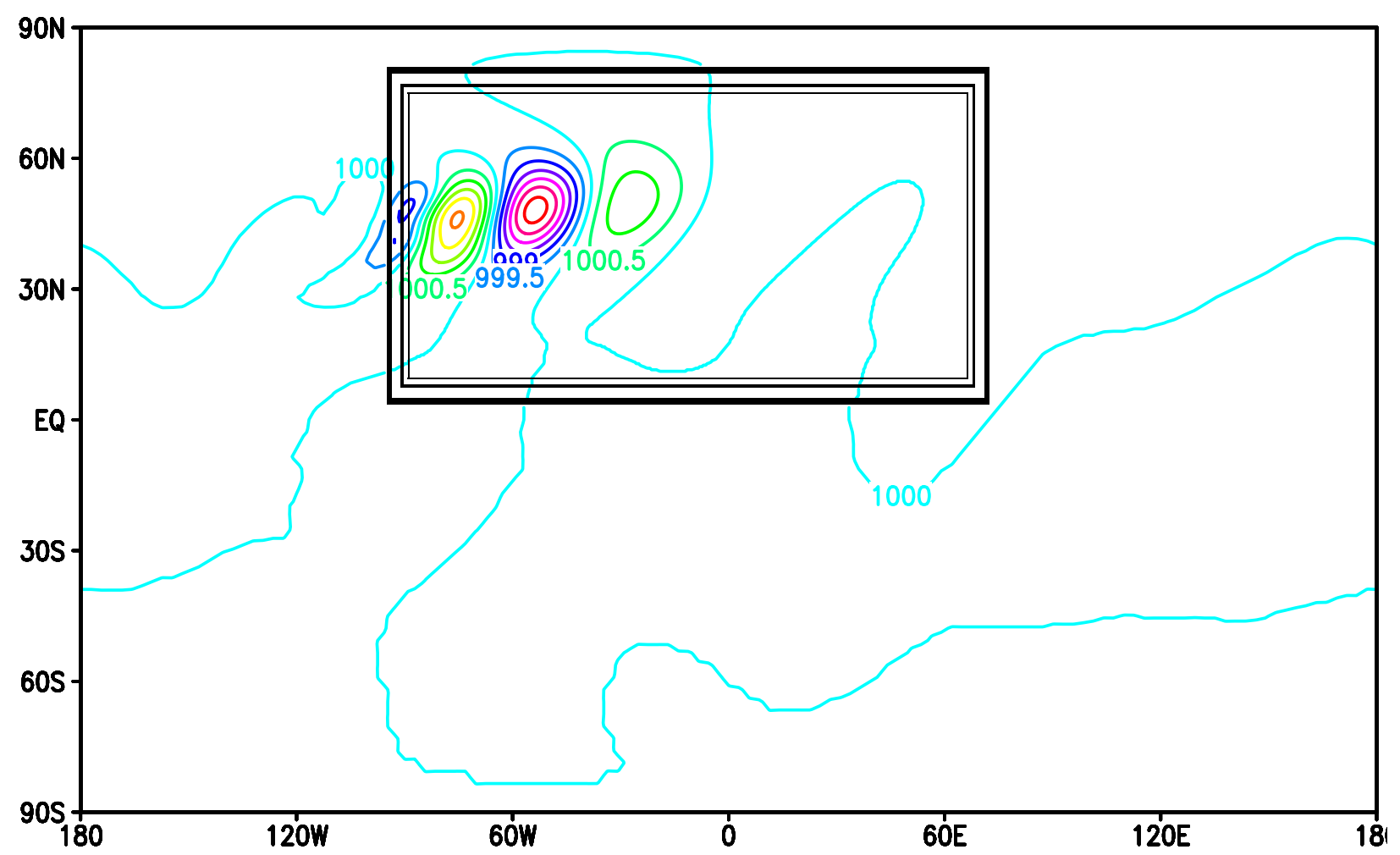

Figura 3.10: Localizaçao dos refinamentos locais e pressão de superfície inicial para o teste da onda baroclínica com refinamentos locais. 


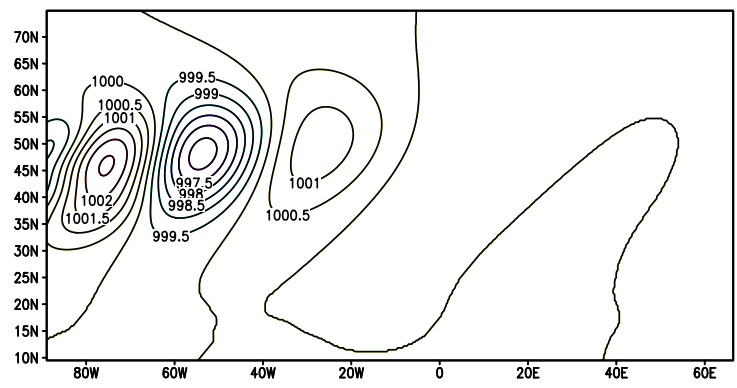

(a) Condição inicial (dia 5)

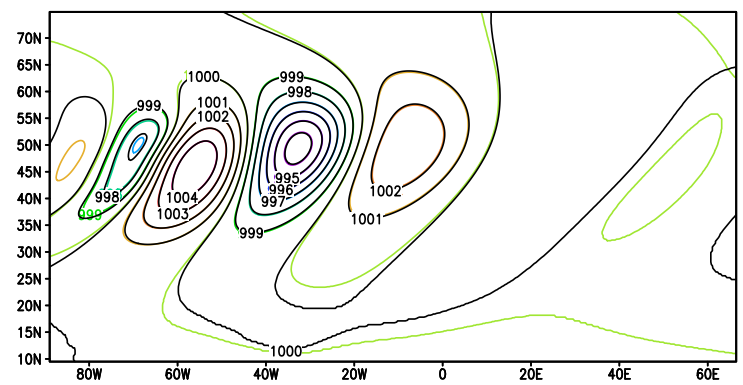

(c) Dia 1

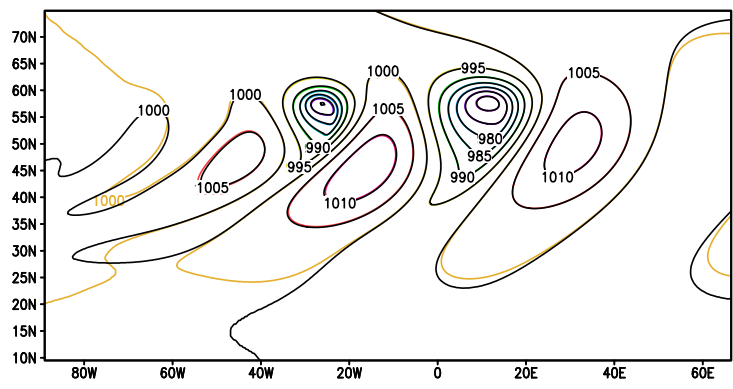

(e) Dia 3

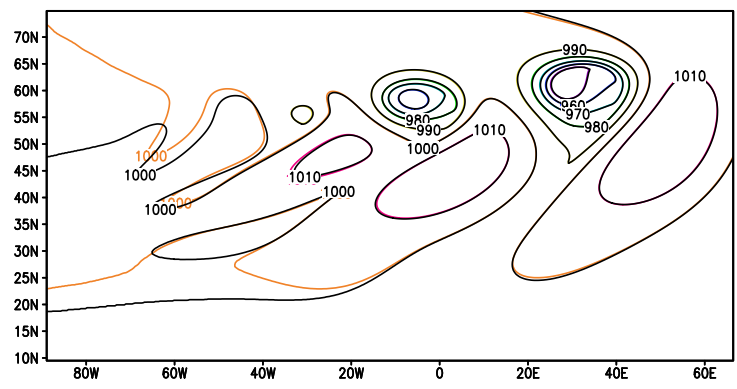

(g) Dia 4

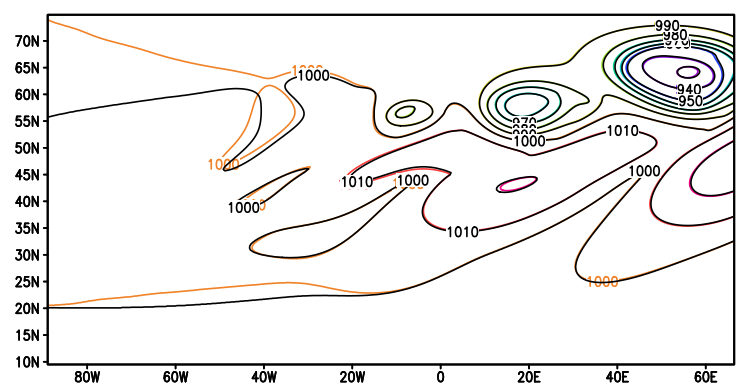

(i) Dia 5

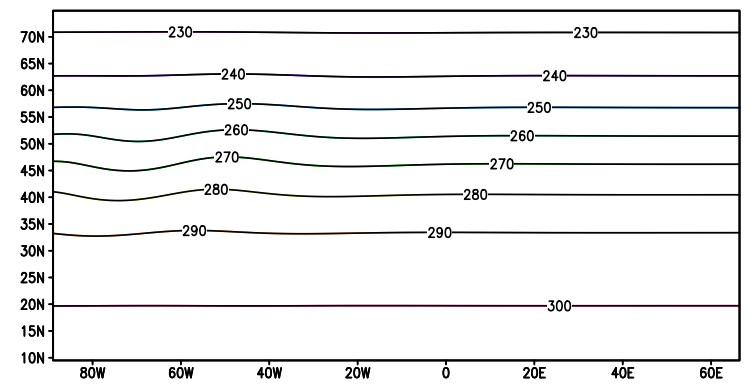

(b) Condição inicial (dia 5)

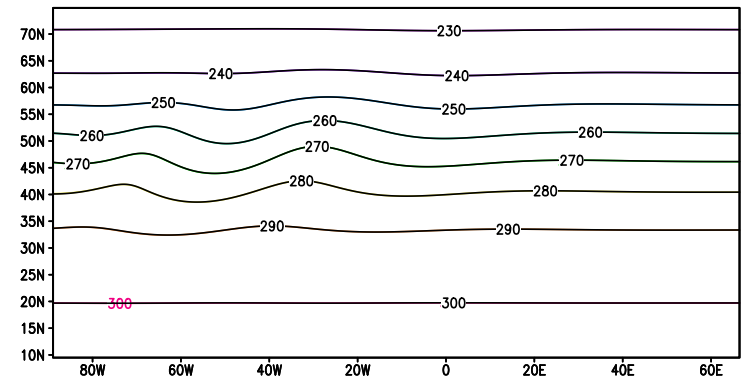

(d) Dia 1

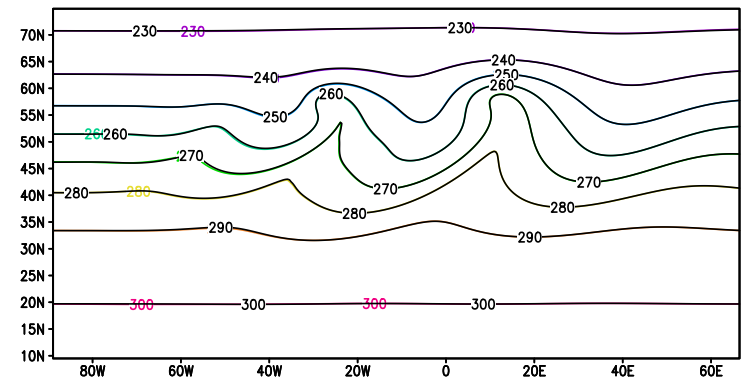

(f) Dia 3

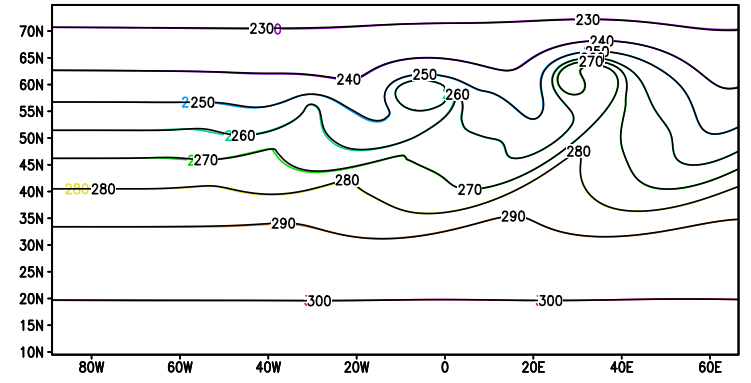

(h) Dia 4

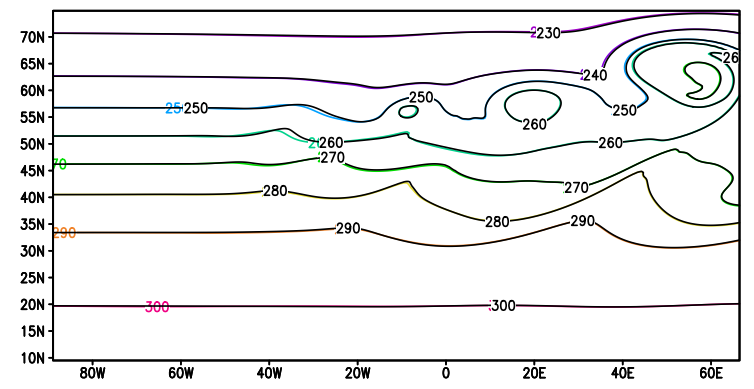

(j) Dia 5

Figura 3.11: Pressão de superfície (esquerda) e temperatura em 850hPa (direita) para o teste da onda baroclínica. Em cores os resultados do refinamento local mais fino e em preto os da malha de referência. 


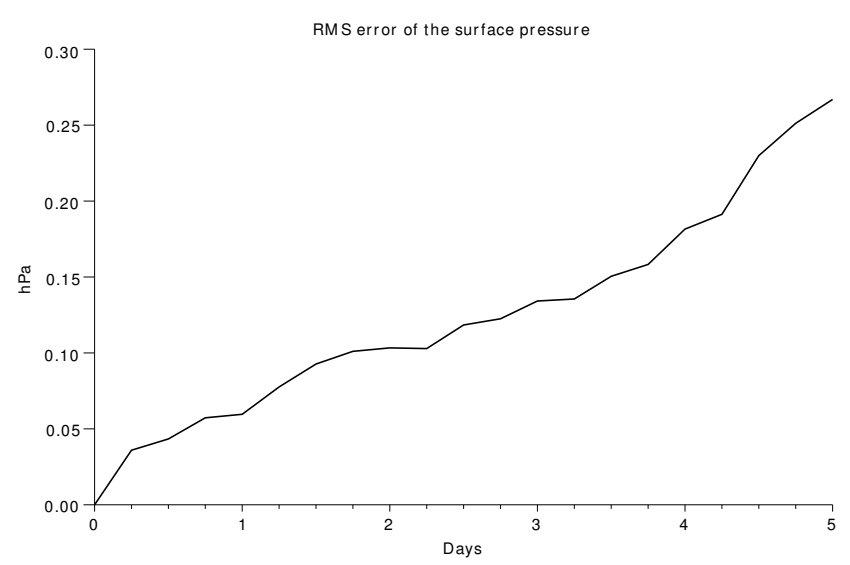

Figura 3.12: Norma $_{2}$ (hPa) sobre a área do refinamento local mais fino, da diferença da pressão de superfície entre a solução de referencia na malha uniforme e a solução calculada empregando refinamento local

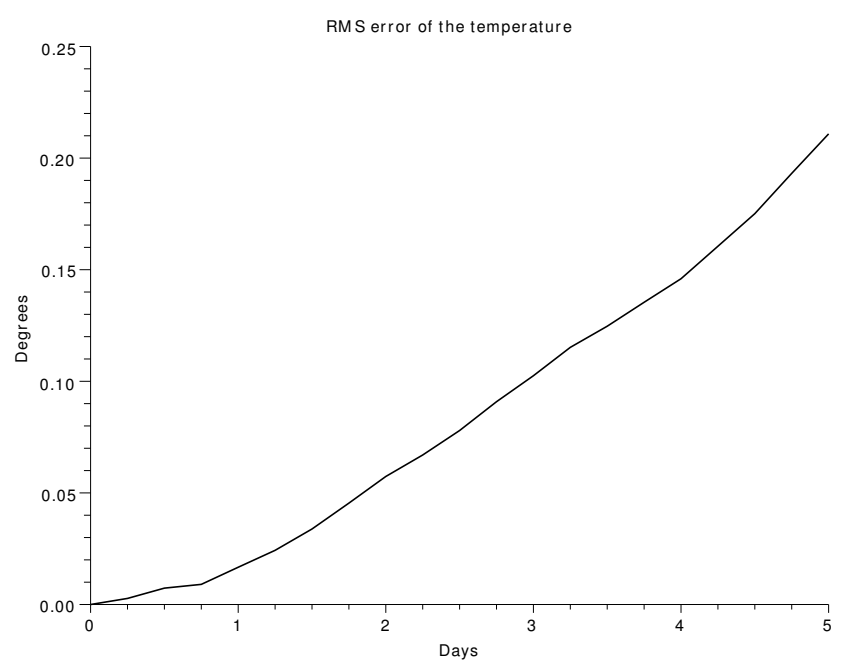

Figura 3.13: Norma $_{2}\left({ }^{\circ} \mathrm{K}\right)$ sobre a área do refinamento local mais fino, da diferença da temperatura entre a solução de referencia na malha uniforme e a solução calculada empregando refinamento local 


\subsubsection{Teste com dados reais e refinamentos locais}

Nesta etapa da validação utilizamos dados reais e malhas com refinamentos locais. Os dados reais, correspondentes ao dia 27 de março de 2004, tem uma resolução horizontal de $512 \times 256$ pontos e 42 níveis verticais e foram interpolados cúbicamente para uma malha uniforme com resolução horizontal de $1024 \times 513$ pontos e 42 níveis na vertical. Utilizamos estes dados interpolados como condição inicial para integrar o nosso modelo por 5 dias, com um tamanho de passo de 450s, obtendo a nossa solução de referência. Para a integração do modelo utilizando malhas refinadas, consideramos uma malha global e três refinamentos locais, cujas dimensões e posições são determinadas pelos valores da seguinte figura:

! Number of refinement levels

nref $=3$

! Grid locations

imin_ref $=0,47, \quad 3, \quad 3$

imax_ref $=127,86, \quad 75,141$

jmin_ref $=0,36,3,3$

jmax_ref $=64,56, \quad 37,65$

Figura 3.14: Configuração das malhas para o teste com dados reais e refinamentos locais.

Analogamente ao teste anterior, da Figura 3.14 observamos que a resolução do refinamento mais fino coincide com a resolução da nossa malha de referência, o que fácilmente permite a comparação das soluções obtidas em ambas as malhas. Como condição inicial para o conjunto de malhas tomamos os campos da nossa solução de referência após um dia de integração e os interpolamos cúbicamente para cada uma das malhas do conjunto (para o refinamento mais fino fazemos uma cópia simples). A Figura 3.15 mostra a localização dos refinamentos locais e a pressão de superfície inicial do teste. Já Figura 3.16 mostra a pressão de superfície (à esquerda) e a altura geopotencial em 500hPa (à direita) para o refinamento mais fino (em cores) e para a malha de referência (em preto). Observamos que no dia 1 os valores da pressão de superfície e da altura geopotencial do refinamento estão bem próximos dos da malha de referência (Figuras 3.16c e 3.16d), mas pequenas diferenças podem ser observadas na altura geopotencial ao noroeste. No dia 2, as diferenças na pressão de superfície aumentam um pouco mas os centros das altas e baixas continuam sendo bem representadas no refinamento (Figura 3.16e). Já para a altura geopotencial observamos diferenças maiores embora não sejam muito grandes (Figura 3.16f). No dia 3 a diferença entre os valores da pressão de superfície para o refinamento e a malha de referência aumentam mas a maioria dos centros de altas e baixas coincidem (Figura 3.16g) enquanto observamos diferenças maiores na altura geopotencial (Figura 3.16h), embora a estrutura da mesma seja mantida, indicando que fenômenos mal resolvidos nas malhas grossas tem contaminado o refinamento. No dia 4 alguns centros de altas e baixa pressão ainda são bem representados no refinamento (Figura 3.16i) mas a altura geoptencial exibe evidente diferenças (Figura 3.16j). 


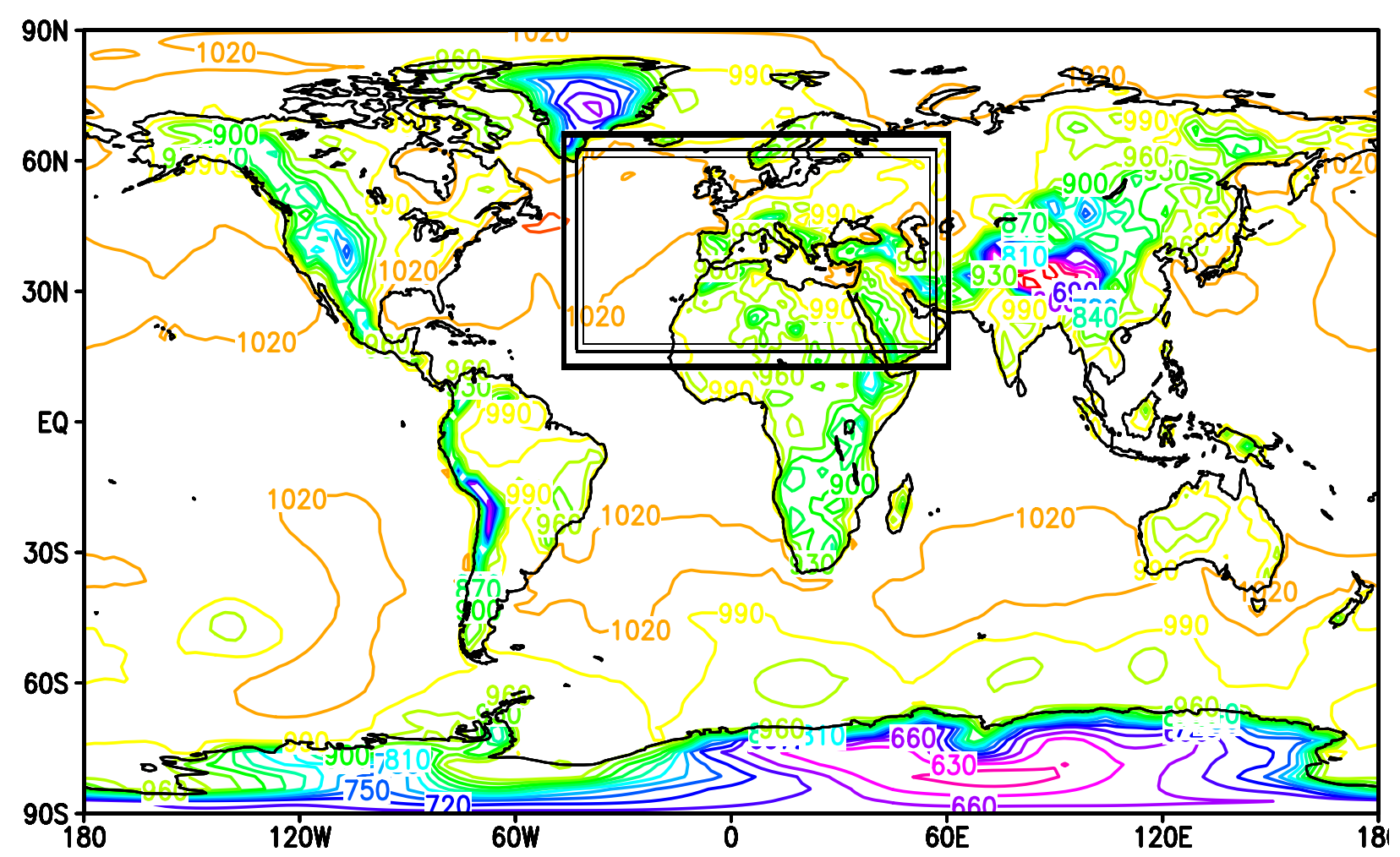

Figura 3.15: Localizaçao dos refinamentos locais e pressão de superfície inicial para o teste com dados reais e refinamentos locais. 


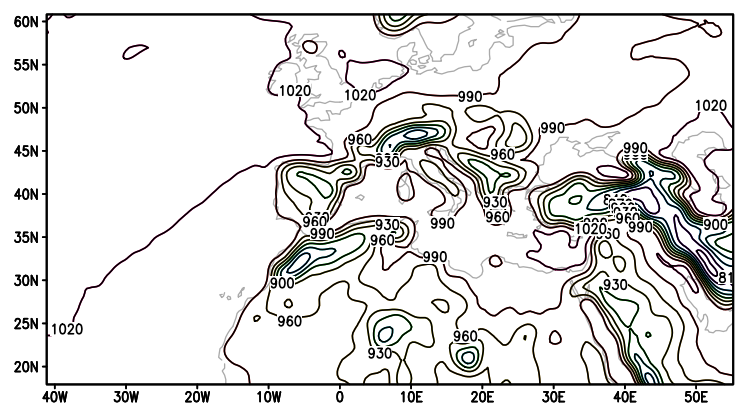

(a) Condiçẫo inicial (dia 1)

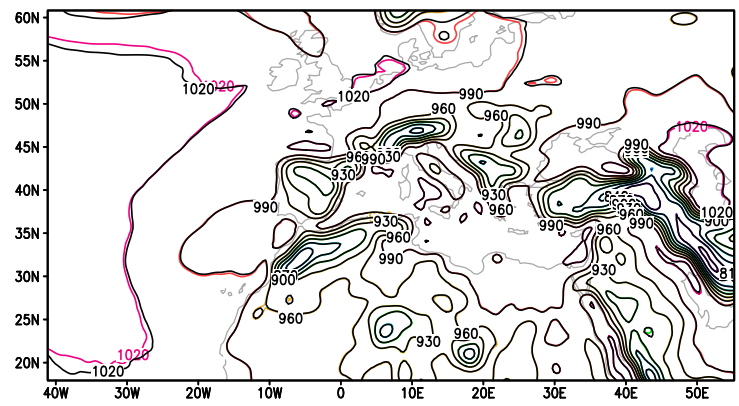

(c) Dia 1

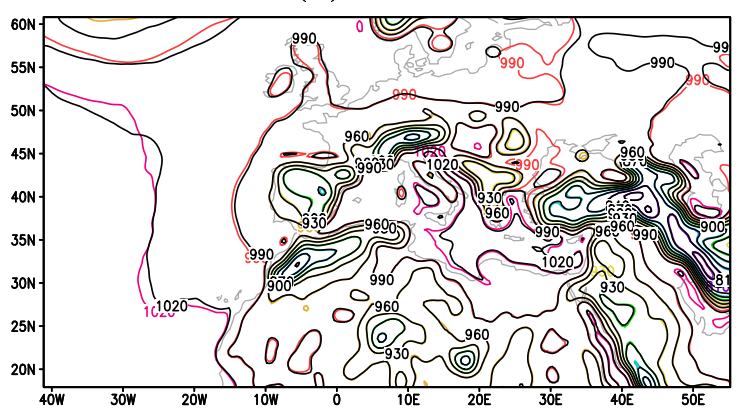

(e) Dia 2

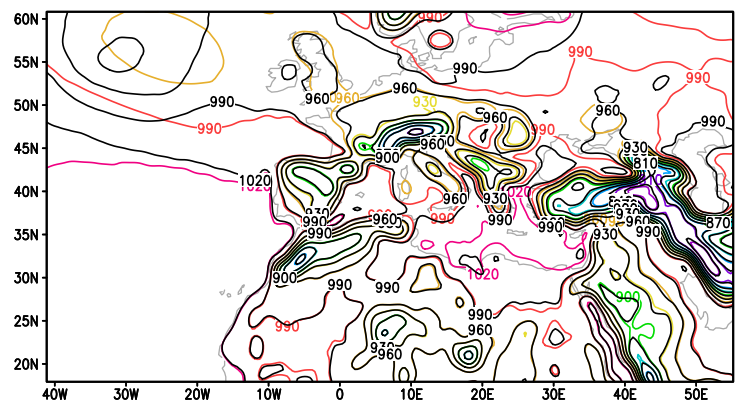

(g) Dia 3

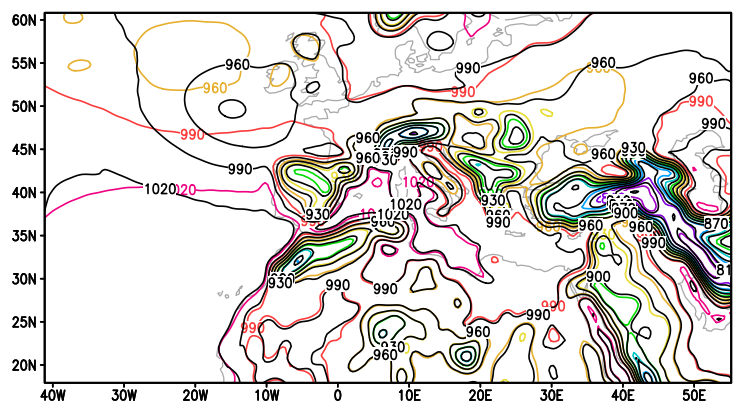

(i) $\operatorname{Dia} 4$

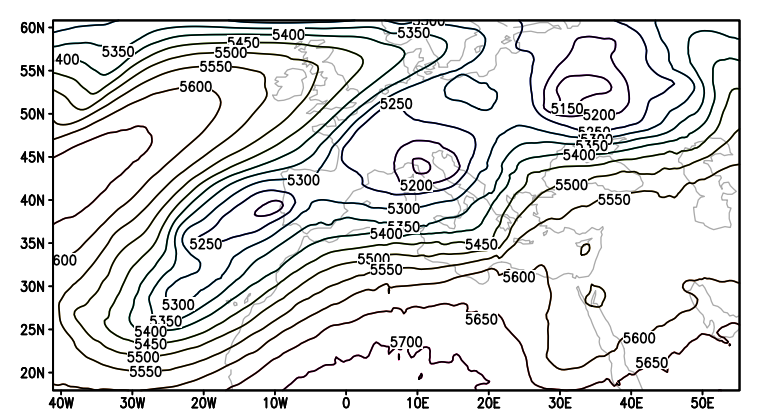

(b) Condição inicial (dia 1)

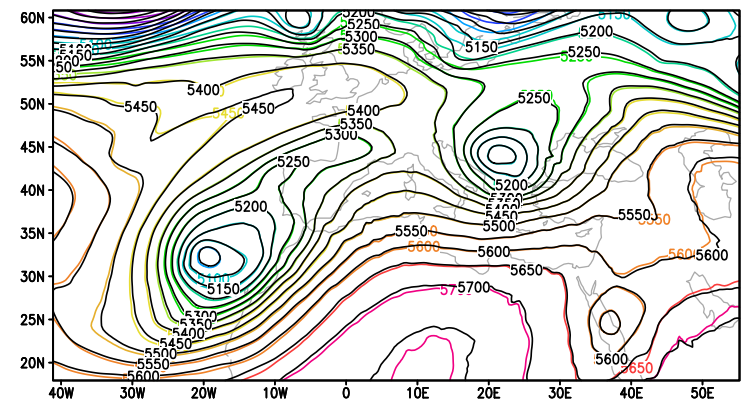

(d) Dia 1

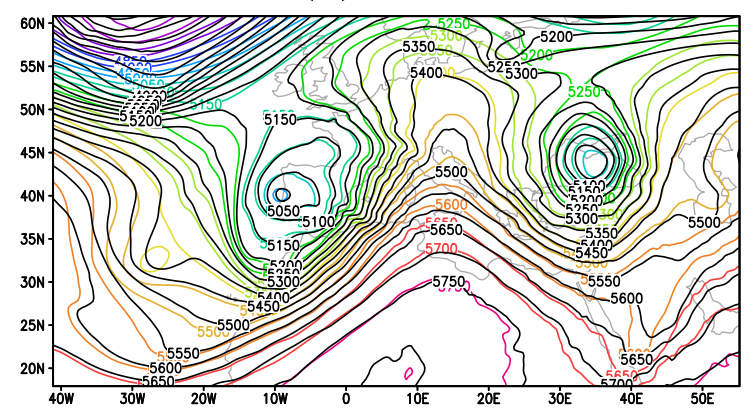

(f) Dia 2

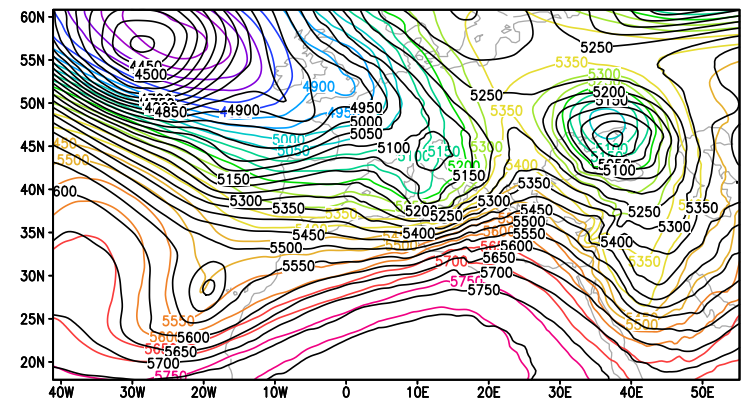

(h) Dia 3

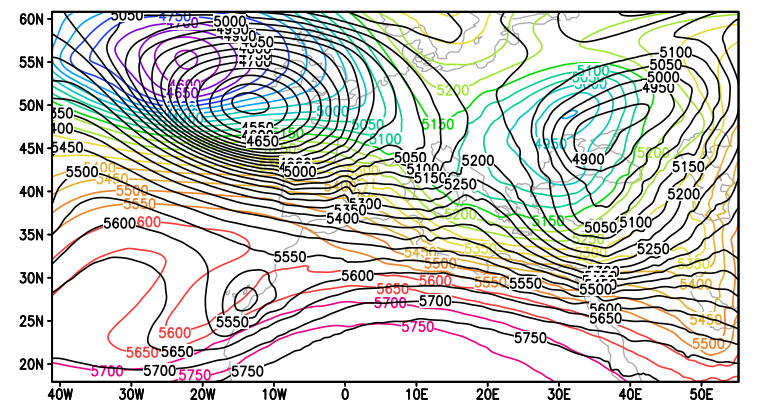

(j) Dia 4

Figura 3.16: Pressão de superfície (esquerda) e altura geopotencial em 500hPa (direita) para o teste com dados reais. Em cores os resultados do refinamento local mais fino e em preto os da malha de referência. 
Finalizando esta etapa, as figuras 3.17 e 3.18 mostram, respectivamente, a norma $l_{2}$ do erro da pressão de superfície e da temperatura em $\sigma=0.865$, em relação à solução de referência.

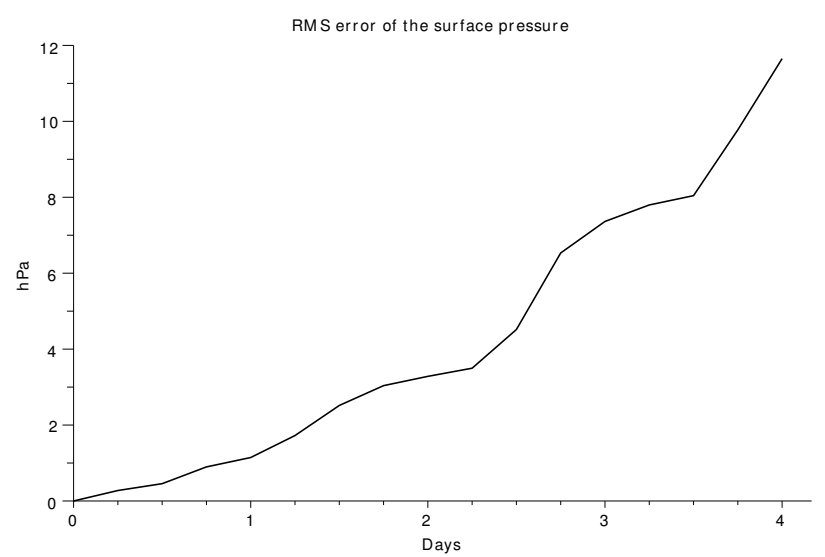

Figura 3.17: Norma $_{2}$ (hPa) da diferença da pressão de superfície entre o refinamento local mais fino e a resolução de referência $1024 \times 513 \times 26$, ao longo do tempo.

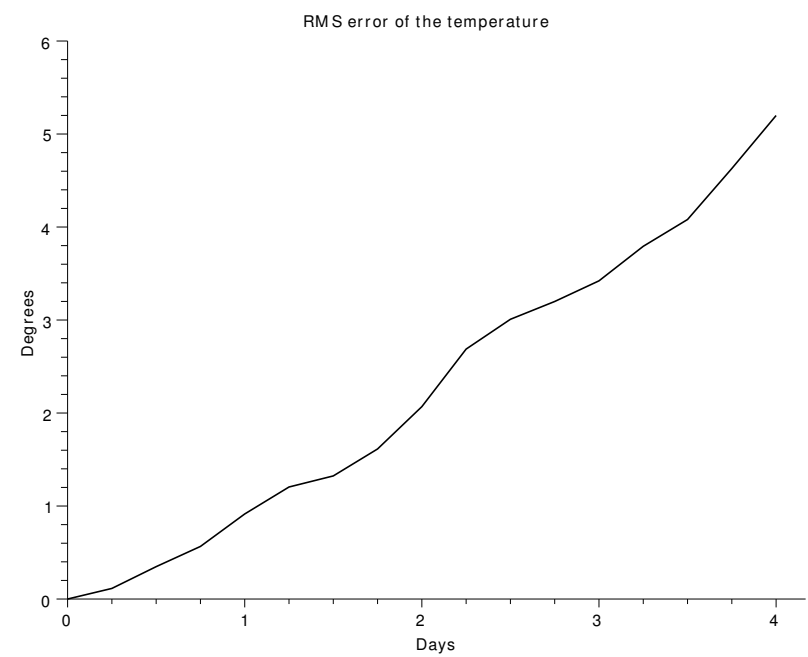

Figura 3.18: Norma $l_{2}\left({ }^{\circ} \mathrm{K}\right)$ da diferença da temperatura entre o refinamento local mais fino e a resolução de referência $1024 \times 513 \times 26$, ao longo do tempo. 


\subsection{Teste de desempenho}

Uma vez validado o modelo, o instrumentalizamos para que calcule o tempo empregado em várias etapas do mesmo. Distinguimos as seguintes:

1. Cálculo dos termos das equações.

2. Transporte Semi-Lagrangiano.

3. Cálculo do lado direito da equação do geopotencial generalizado.

4. Resolução da equação do geopotencial generalizado pelo método multigrid.

5. Cálculo dos novos valores dos campos

Para cada uma destas etapas calculamos

a) Tempo total utilizado

b) Tempo de comunicação

c) Tempo de cómputo nos pontos inativos

d) Tempo de cómputo nos pontos ativos

Adicionalmente calculamos o tempo utilizado na transposição dos dados, o tempo de I/O, o tempo total das iterações e o tempo total utilizado por cada processador. No final da integração do modelo, cada processador envia seus tempos ao servidor de I/O, que os salva em um arquivo.

Utilizamos o cluster tupa $a^{3}$ do CPTEC para executar nosso modelo, que consiste de um supercomputador Cray XE6, com sistema de interconexão Gemini, operando em sua capacidade total com 14 gabinetes, 1.304 nós computacionais e 31.296 processadores. Salientamos ainda que a nosso pedido o Prof. Saulo Barros gentilmente realizou o teste descrito a seguir no referido cluster.

O teste executado nesta máquina consiste na previsão de um dia do teste da onda baroclínica, utilizando um tamanho de passo do tempo $\Delta t=112.5 \mathrm{~s}$ e 32 níveis na vertical, totalizando 768 iterações. A configuração horizontal das malhas consiste em uma malha base contendo $256 \times 129$ pontos e quatro refinamentos locais, como é mostrada na Figura 3.19 .

Executamos o teste para 32, 64, 128, 256, 512 e 1024 processadores. A Tabela 3.3 mostra a distribuição de processadores, por região, para os números de processadores acima mencionado. Observamos a diminuição do desbalancemanto de carga, mostrado em porcentagem e em relação ao valor ideal, conforme aumenta o número de processadores, justificado pela diminuição no número de pontos por processador. Observamos também que a malha fina responde por aproximadamente $95 \%$ do total de pontos e que a zona de transição, com poucos pontos, utiliza mais de um processador somente a partir de 128 processadores.

\footnotetext{
${ }^{3}$ http://www.cptec.inpe.br/supercomputador/
} 
! Number of refinement levels

nref $=4$

! Grid locations

imin_ref $=0,60,3,3,3$

imax_ref $=255,114,105,201,393$

jmin_ref $=0,17,3,3,3$

jmax_ref $=128, \quad 82,127,245,481$

Figura 3.19: Configuração da malha base e 4 refinamentos locais utilizadas no teste de desempenho.

\begin{tabular}{|c|c|c|}
\hline região & $\#$ pts & $\%$ \\
\hline grossa & 29632 & 3.79 \\
\hline trans & 9276 & 1.18 \\
\hline fina & 743945 & 95.03 \\
\hline total & 782853 & 100 \\
\hline
\end{tabular}

(a) Número de pontos por região

\begin{tabular}{|c|c|c|}
\hline $\mathrm{npr} / \mathrm{reg}$ & $\mathrm{npts} / \mathrm{pr}$ & \% des \\
\hline 2 & 14816 & 39.44 \\
\hline 1 & 9276 & 62.09 \\
\hline 29 & 25654 & -4.86 \\
\hline $\mathbf{3 2}$ & 24465 & - \\
\hline
\end{tabular}

(b) 32 processadores

\begin{tabular}{|c|c|c|}
\hline $\mathrm{npr} / \mathrm{reg}$ & $\mathrm{npts} / \mathrm{pr}$ & $\%$ des \\
\hline 5 & 5927 & 3.10 \\
\hline 2 & 4638 & 24.17 \\
\hline 121 & 6149 & -0.53 \\
\hline $\mathbf{1 2 8}$ & 6117 & - \\
\hline
\end{tabular}

(d) 128 processadores

\begin{tabular}{|c|c|c|}
\hline $\mathrm{npr} / \mathrm{reg}$ & $\mathrm{npts} / \mathrm{pr}$ & \% des \\
\hline 20 & 1482 & 3.10 \\
\hline 7 & 1326 & 13.33 \\
\hline 485 & 1534 & -0.32 \\
\hline $\mathbf{5 1 2}$ & 1530 & - \\
\hline
\end{tabular}

(f) 512 processadores

\begin{tabular}{|c|c|c|}
\hline $\mathrm{npr} / \mathrm{reg}$ & $\mathrm{npts} / \mathrm{pr}$ & \% des \\
\hline 3 & 9878 & 19.25 \\
\hline 1 & 9276 & 24.17 \\
\hline 60 & 12400 & -1.37 \\
\hline $\mathbf{6 4}$ & 12233 & - \\
\hline
\end{tabular}

(c) 64 processadores

\begin{tabular}{|c|c|c|}
\hline npr/reg & npts/pr & \% des \\
\hline 10 & 2964 & 3.10 \\
\hline 4 & 2319 & 24.17 \\
\hline 242 & 3075 & -0.53 \\
\hline $\mathbf{2 5 6}$ & 3059 & - \\
\hline
\end{tabular}

(e) 256 processadores

\begin{tabular}{|c|c|c|}
\hline npr/reg & npts/pr & \% des \\
\hline 39 & 760 & 0.62 \\
\hline 13 & 714 & 6.67 \\
\hline 972 & 766 & -0.12 \\
\hline $\mathbf{1 0 2 4}$ & 765 & - \\
\hline
\end{tabular}

(g) 1024 processadores

Tabela 3.3: Distribuição de processadores por região

A Tabela 3.4 mostra os tempos totais obtidos na execução do nosso teste para os números de processadores mencionados. Baseado nestes valores, as Figuras 3.20 e 3.20 mostram respectivamente o speedup e a eficiência alcanzados [53], em relação ao tempo empregado para 32 processadores, de onde podemos observar o bom desempenho do nosso modelo, que para 
1024 processadores atingiu um speedup de 18.97 e uma eficiênca de $59.28 \%$.

\begin{tabular}{|c|c|}
\hline$\#$ procs & Tempo (s) \\
\hline 32 & 3229.34 \\
\hline 64 & 1840.20 \\
\hline 128 & 900.64 \\
\hline 256 & 496.35 \\
\hline 512 & 274.87 \\
\hline 1024 & 170.23 \\
\hline
\end{tabular}

Tabela 3.4: Tempos totais dos testes realizados no cluster tupã

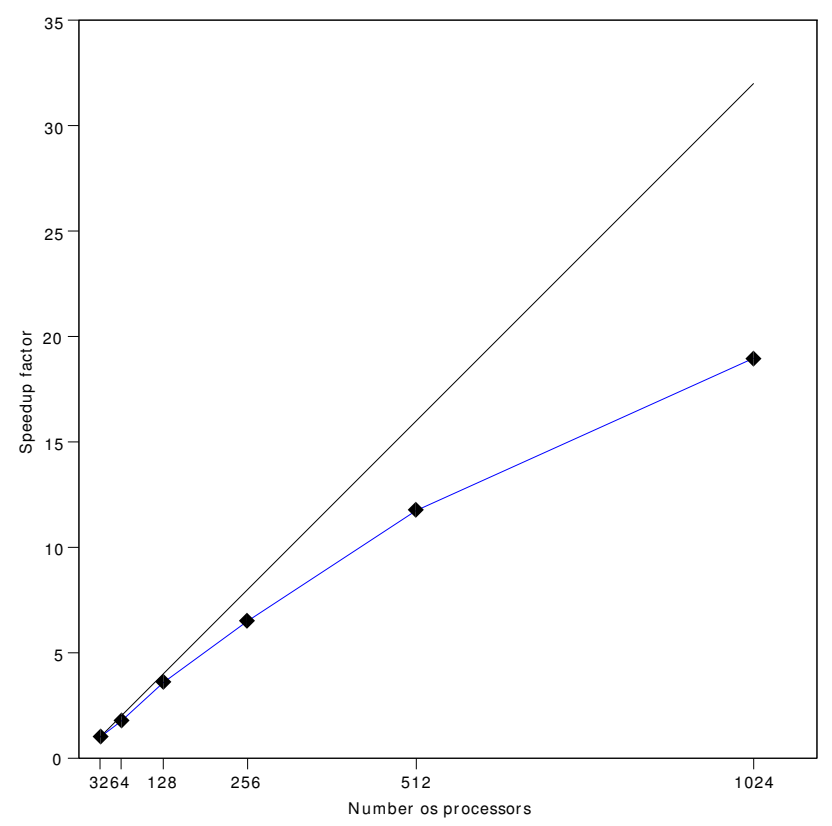

Figura 3.20: Speedup obtido pela bateria de testes no cluster tupã

Como dizemos anteriormente, a cada iteração o modelo efetua um conjunto específico de tarefas, as quais compreendem o cálculo dos termos das equações, o transporte SemiLagrangiano, o cálculo do lado direito da equação do geopotencial generalizado, a solução desta equação pelo método multigrid, o cálculo dos novos valores dos campos a partir desta solução e a transposição dos dados antes e depois da aplicação do método multigrid. A Figura 3.22 mostra os tempos mínimo, médio e máximo empregados pelos processadres para cada uma destas tarefas assim como o tempo total, para a execução do teste com 32 , $64,128,256,512$ e 1024 processadores. Observamos que a tarefa que consome mais tempo é a do transporte Semi-Lagrangiano e que conforme aumenta o número de processadores aumenta o desbalanceamento de carga para esta tarefa, evidenciado pelo fato do valor médio estar mais próximo do valor mínimo que do valor máximo. Observamos também que com o 


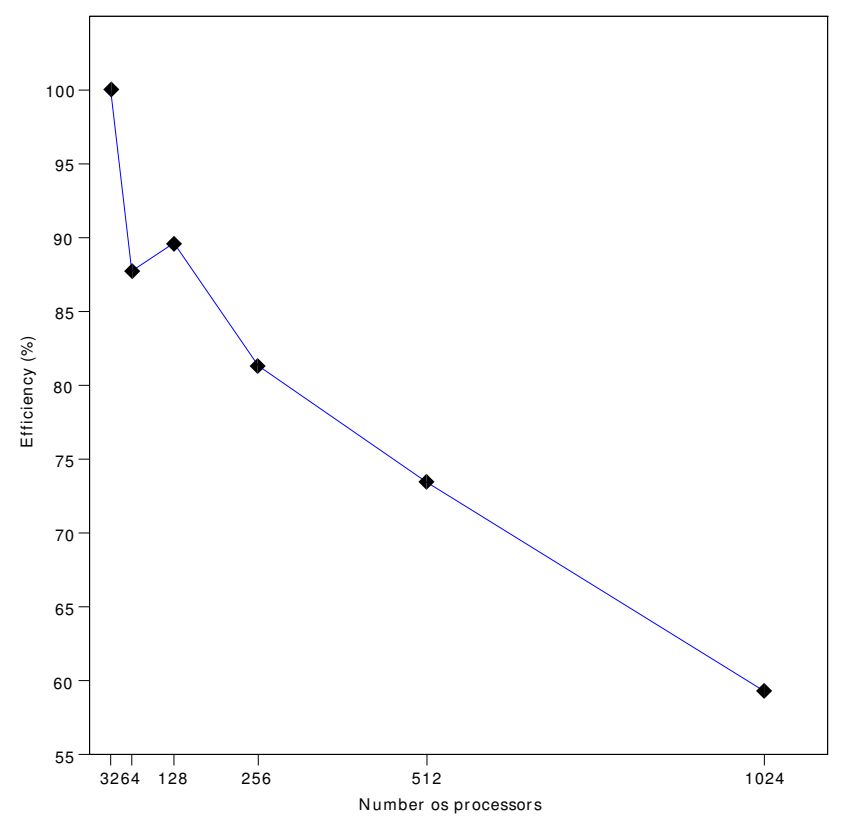

Figura 3.21: Eficiência obtida pela bateria de testes no cluster tupã

aumento no número de processadores este tempo diminui ligeiramente quando comparado ao tempo total de integração, e que por sua vez o tempo da transposição dos dados aumenta. Estes comportamentos podem ser explicados pelo fato de que, com um número maior de processadores, diminui o número de pontos em que cada processador trabalha e aumenta o número de mensagens a serem comunicados.

Com o intuito de entender melhor o tempo gasto pelo transporte Semi-Lagrangiano, separamos os processadores de acordo com a região em que atuam. A Figura 3.23 mostra os tempos mínimos, médios e máximos dos processadores segundo a região em que atuam, assim como o tempo total, para o teste de desempenho com 1024 processadores. Observamos que nos processadores agindo nas regiões grossa e de transição o tempo médio é maior que o daqueles agindo na malha fina e mais próximo dos seus respectivos valores máximos, indicando um bom balanceamento de carga. Por outro lado, a média dos processadores agindo na malha fina, está mais próximo do valor mínimo, evidenciando um desbalanceamento de carga nesta região. Uma possível explicação para isto pode ser o fato de que a decomposição do domínio é feita considerando os pontos da malha F (lembremos que utilizamos três tipos de malhas) e que para os refinamentos locais foram adicionadas duas colunas de pontos para a malha U (uma à esquerda e a outra à direita), duas filas para a malha $\mathrm{V}$ (uma na parte inferior e a outra na parte superior) e que os processadores que agem na fronteira desses refinamentos assumen estas filas/colunas adicionais. Ainda mais, estes processadores necessitam comunicar dados com malhas de resolução diferente e realizar mais cálculos sobre os pontos inativos, contribuindo assim com o desbalanceamento de carga. 


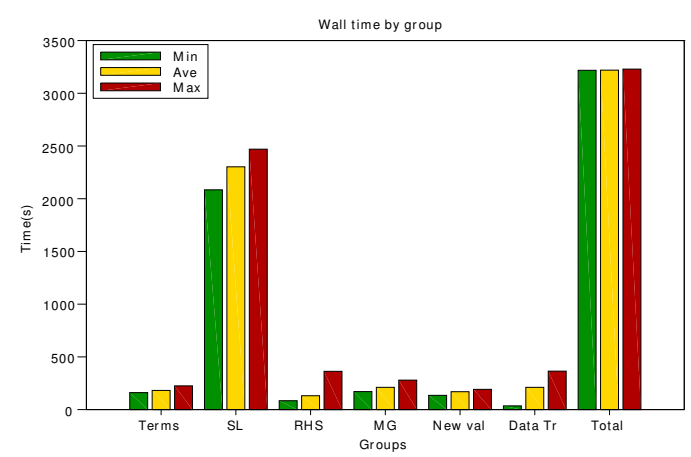

(a) 32 processadores

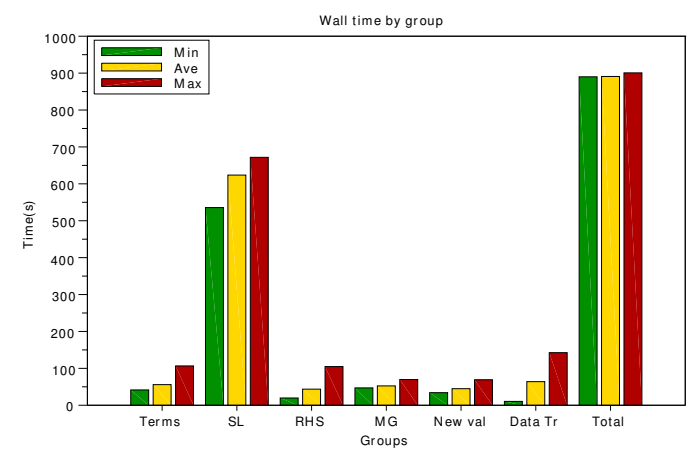

(c) 128 processadores

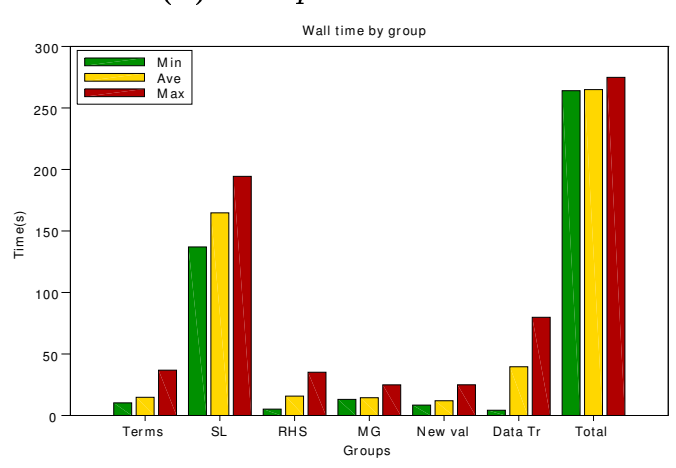

(e) 512 processadores

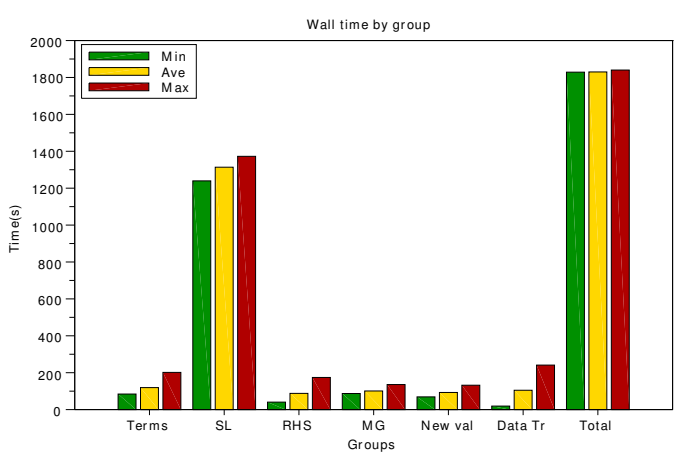

(b) 64 processadores

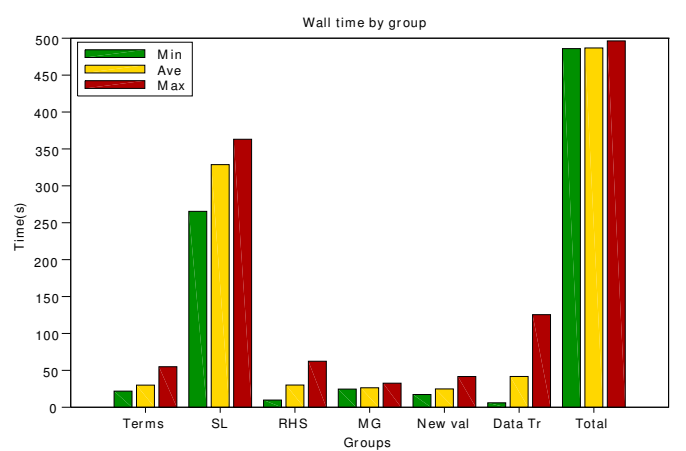

(d) 256 processadores

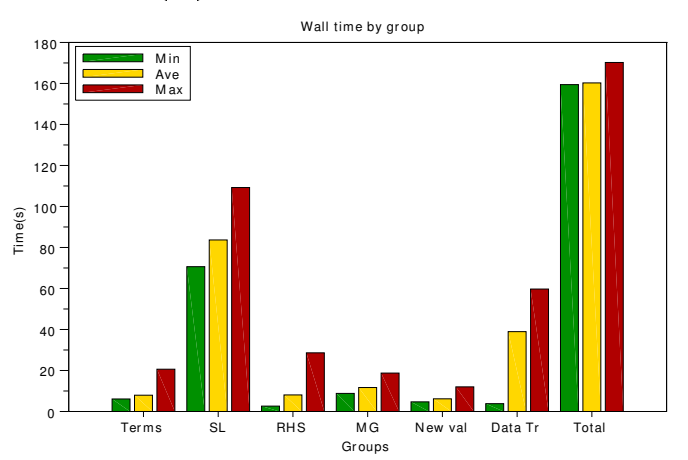

(f) 1024 processadores

Figura 3.22: Tempos mínimos, médios e máximos das principais etapas ocorridas durante a integração do modelo, para o teste de desempenho.

Para confirmar as nossas hipóteses, a Figura 3.24 mostra os os tempos mínimos, médios e máximos gastos no transporte Semi-Lagrangiano pelos processadores da malha fina, separados em dois grupos: os que agem na fronteira e os que agem no interior da malha. Adicionalmente são mostrados os tempos mínimos, médios e máximos de todos os processadores para essa malha. Observamos que os três valores para os processadores agindo no interior da malha são menores que os respectivos valores para os processadores da fronteira.

Adicionalmente, a Figura 3.25 mostra os tempos totais, de comunicação, de cómputo nos pontos inativos e de cómputo nos pontos ativos gastos pelos processadores da malha fina no transporte Semi-Lagrangiano, divididos em processadores interiores e de fronteira. Observamos que o tempo total gasto pelos processadores na fronteira é em geral maior que o dos processadores interiores (Figura 3.25a). Observamos também que o tempo de 


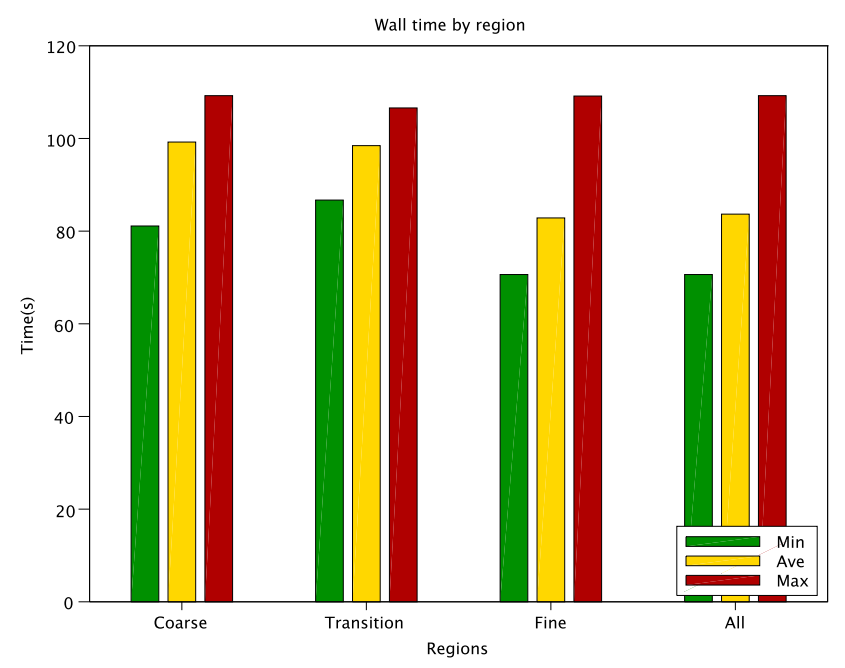

Figura 3.23: Tempos mínimos, médios e máximos, segundo a região e total, gastos no transporte Semi-Lagrangiano, para o teste de desempenho com 1024 processadores

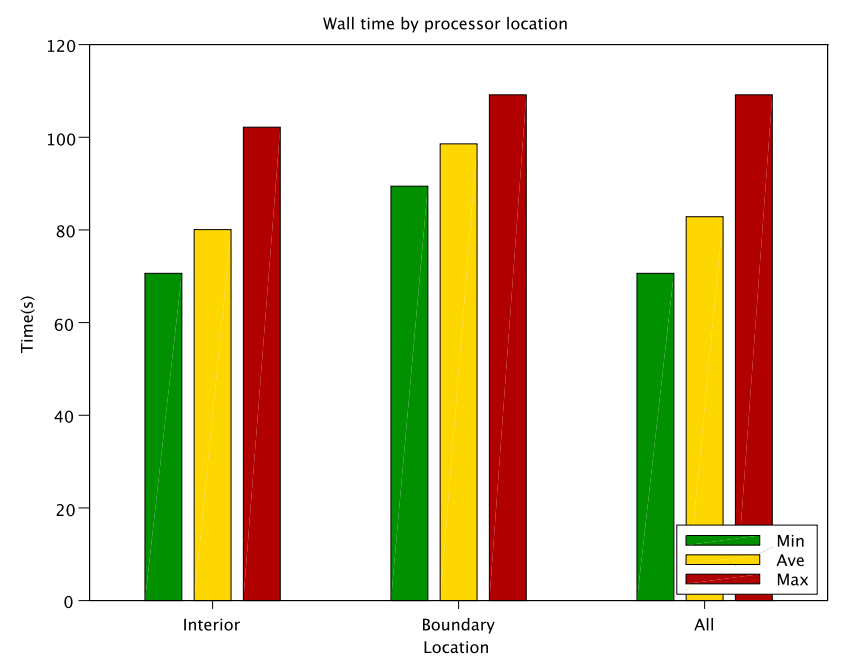

Figura 3.24: Tempos minimos, médios e máximos para os processadores da malha fina, separados em interiores, de fronteira e valores totais.

comunicação utilizado nesta etapa representa uma considerável porcentagem, em torno de $30 \%$, do tempo total e que determina o padrão oscilatório exibido neste último (Figura 3.25b). Adicionalmente observamos que o tempo utilizado no cálculo dos pontos inativos, embora seja extremamente pequeno em relação ao tempo total, é muito maior para os processadores agindo na fronteira da malha do que para os que agem no interior da mesmai (Figura 3.25c). Como mencionamos anteriormente, os processadores da fronteira trocam informações com processadores que trabalham em malhas com outras resoluções e necessitam de um trabalho maior nos pontos inativos, explicando este comportamento. Finalmente, observamos que ambos os processadores interiores e de fronteira exibem um mesmo padrão no tempo de cómputo nos ponto ativos (Figura 3.25d), com exceção de alguns processadores do último 
tipo.

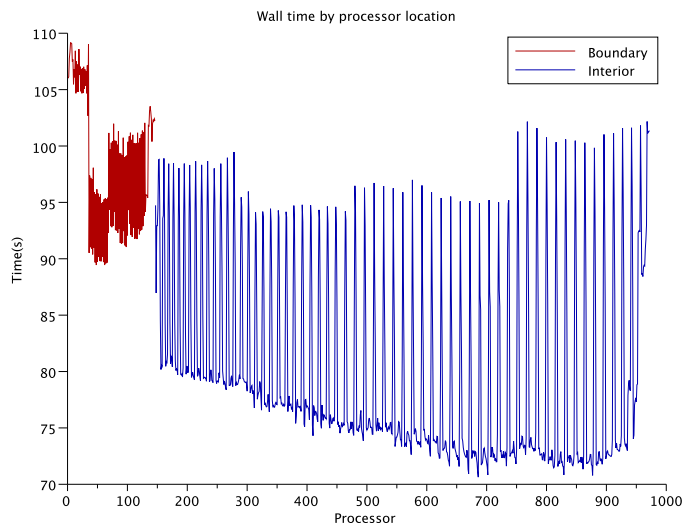

(a) Tempo total

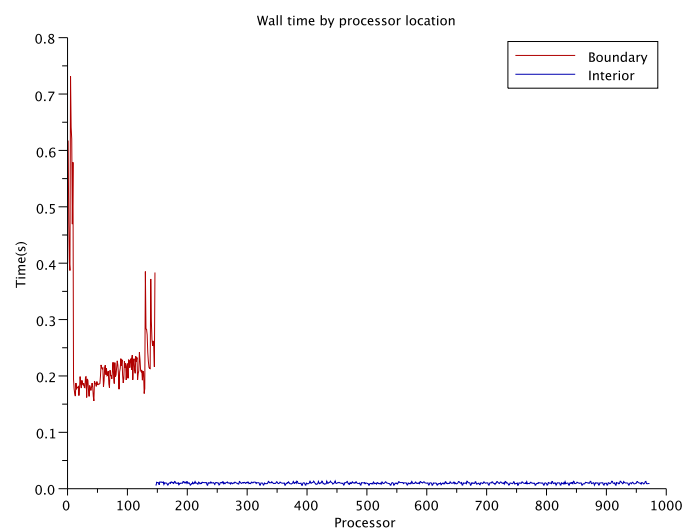

(c) Tempo de cómputo nos pontos inativos

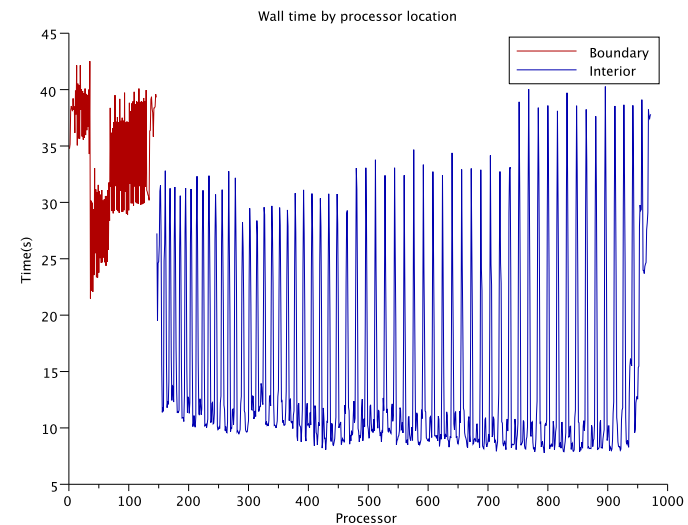

(b) Tempo de comunicação

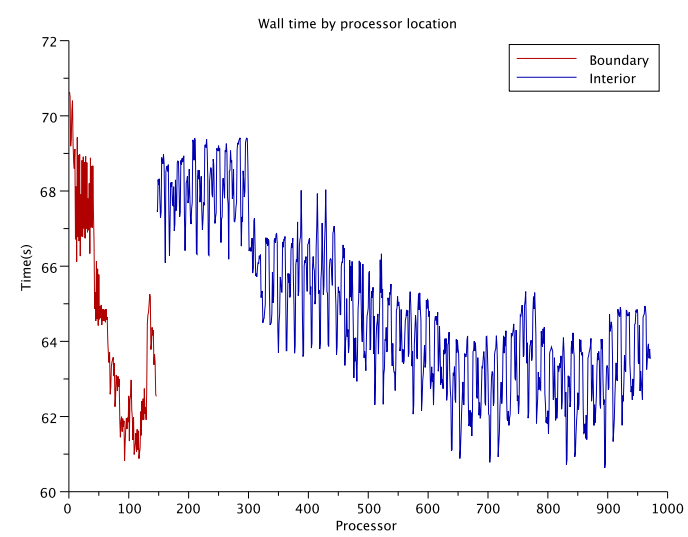

(d) Tempo de cómputo nos pontos ativos

Figura 3.25: Tempos total, de comunicação, de cómputo em pontos inativos e de cómputo em pontos ativos para o transporte Semi-Lagrangiano, para 1024 processadores.

Finalmente, a Figura 3.26 mostra a porcentagem que os tempos mínimos, médios e máximos utilizados na transposição dos dados representam do tempo total médio, para o nosso teste de desempenho e número de processadores igual a 32, 64, 128, 256, 512 e 1024. Observamos que dita porcentagem aumenta conforme aumenta o número de processadores (note que para 1024 processadores representa, em média, 25\% do tempo total). A explicação para este comportamento reside em que com um número maior de processadores, a quantidade de pontos por processador diminui e na redistribuição global dos dados teremos um número maior de mensagens a comunicar, cada uma destas com tamanho menor. Observamos também um considerável desbalanceamento nesta etapa.

Da nossa análise para o nosso teste de desempenho concluímos que o nosso modelo exibe um bom desempenho até os 1024 processadores utilizados. Observamos que os dois processos que consomem mais tempo durante a integração do mesmo são o transporte semiLagrangiano e a transposição dos dados. Com o aumento no número de processadores, o tempo do primeiro diminui ligeiramente enquanto o do segundo aumenta, quando comparados ao tempo total de integração. Observamos também que o tempo da transposição dos 


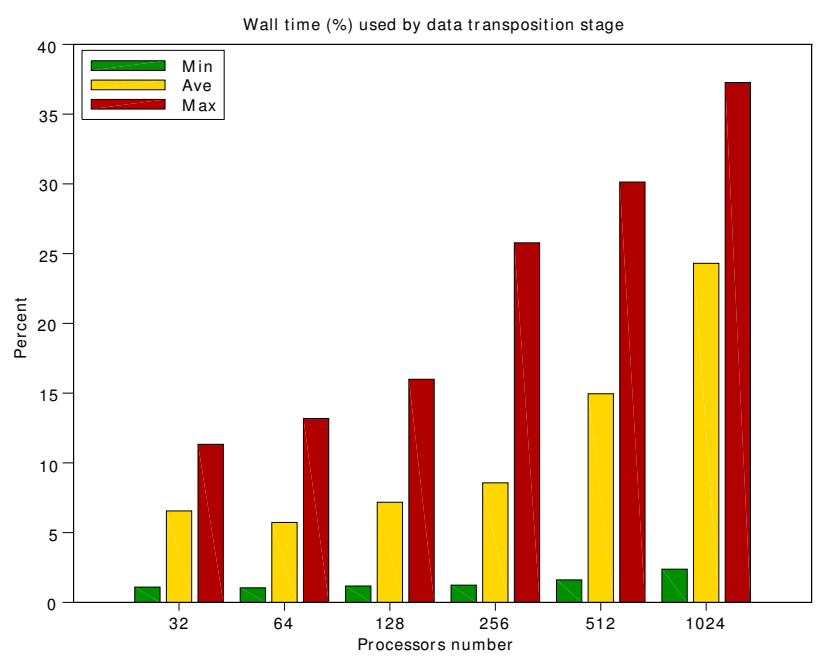

Figura 3.26: Porcentagem dos tempos mínimos, médios e máximos, relativo ao tempo total médio, utilizado na transposição dos dados, para o teste de desempenho.

dados exibe um desbalanço considerável. Esperamos que para números de processadores maiores a 1024 a porcentagem do tempo correspondente à transposição dos dados aumente em relação ao tempo total. Estudos adicionais são necessários para melhorar o desempenho destas duas etapas e consequentemente do modelo. Uma primeira medida a ser tomada seria sobrepor comunicação e computação, que não foi realizado neste trabalho. 


\section{Conclusões}

Neste trabalho propomos uma metodologia de paralelização de um modelo de previsão do tempo semi-Lagrangiano, em diferenças finitas e em malhas localmente refinadas, para máquinas com memória distribuída, tomando como ponto de partida os trabalhos desenvolvidos em [16], [28] e [14]. Do primeiro utilizamos a formulação matemática do modelo, do segundo os conceitos de como utilizar malhas com refinamentos locais e do terceiro as ideias sobre como dividir uma malha em faixas (strips) e remendos (patches).

Durante o desenvolvimento da nossa metodologia, nos vimos na necessidade de definir uma estrutura de dados que nos permitisse trabalhar com malhas (lógicamente) irregulares. A definição do descritor de malhas foi fundamental, uma vez que nos permite dividir as malhas entre os processadores de forma a conseguir um bom balancemanto de carga. Ainda mais, como a varredura de uma malha utilizando o descritor de malhas é feita por segmentos (slices), torna-se apropriada sua utlização na exploração do paralelismo de memória compartilhada pois durante a verredura da malha, cada segmento da mesma pode ser atribuído a um núcleo do processador. Mencionamos que, com exceção do método multigrid, o nosso modelo está programado para explorar o paralelismo de memória compartilhada, mas por este motivo neste trabalho esta opção foi desativada.

O nosso modelo possui malhas localmente refinadas, dificultando o processo de divisão horizontal devido à variação na resolução das mesmas. Observamos que naturalmente surgem três regiões: uma grossa, uma fina e uma de transição, em que a maioria dos pontos concentramse nas malhas grossa e fina. Decidimos dividir estas regioes por separado entre um número de processadores proporcional ao número de pontos que cada uma delas contém. Esta forma de divisão pode gerar um desbalanceamento de carga, que diminui conforme o número de processadores aumenta.

Durante a integração do modelo, necessitamos resolver um sistema de equações bidimensionais de tipo Helmholtz a cada iteração pelo método multigrid. Cada uma destas equações, discretizadas por diferenças finitas, resulta em um operador altamente anisotrópico na direção zonal, perto dos pólos, mudando completamente o sentido das independências computacionais. Decidimos mudar o sentido do paralelismo únicamente nesta etapa para resolver eficientemente o sistema acima mencionado pelo método multigrid e utilizamos a estratégia de tranposição de dados para alternar entre as duas formas de paralelismo.

Validamos o nosso modelo e testamos seu desempenho no supercomputador tupã do CPTEC, para 32, 64, 128, 256, 512 e 1024 processadores. Os testes ainda indicam uma boa 
escalabilidade até os 1024 processadores utilizados.

Como trabalhos futuros, do curto ao longo prazo, pretendemos estender o nosso método multigrid para explorar o paralelismo de memória compartilhada e ativar esta opção no modelo completo. Um outro objetivo é acoplar a física ao nosso modelo e realizar testes de desempenho. Objetivos de mais longo prazo são resolver a equação do geopotencial generalizado sem a necessidade de desacoplamento vertical e evoluir o nosso modelo para uma versão não-hidrostática. 


\section{Referências Bibliográficas}

[1] Brazilian Regional Atmospheric Modelling System - BRAMS. http://brams.cptec.inpe.br. 2

[2] Consortium for Small-Scale Modeling - COSMO. http://www.cosmo-model.org. 2

[3] The Global Forecast System (GFS) - Global Spectral Model (GSM). http://www.emc.ncep.noaa.gov/?branch=GFS\&tabdoc. 2

[4] ICOsahedral Nonhydrostatic general circulation model - ICON. http://www.mpimet.mpg.de/en/science/models/icon.html. 2

[5] Message Passing Interface (MPI) forum home page. http://www.mpi-forum.org. 50

[6] Modelo de Circulação Geral Atmosférico - MCGA. http://previsaonumerica.cptec.inpe.br/mod_glb.shtml. 2

[7] Open Multi-Processing home page. http://www.openmp.org. 38

[8] Arakawa, A., And Lamb, V. R. Computational design of the basic dynamical processes of the UCLA general circulation model. Methods in Computational Physics 17 (1977), 173-265. 27, 32

[9] Asselin, R. Frequency filter for time integrations. Monthly Weather Review 100 (1972), 487-490. 57

[10] Bacon, D., Nash'at, A., Boybeyi, Z., Dunn, T., Hall, M., Lee, P., Sarma, R., AND TURner, M. A dynamically adapting weather and dispersion model: the operational multiscale environment model with grid adaptivity (OMEGA). Monthly Weather Review 128 (2000), 2044-2076. 4

[11] Balsara, D. S., AND NorTon, C. D. Highly parallel structured adaptive mesh refinement using parallel language-based approaches. Parallel Computing 27 (2001), 37-70. 38

[12] Barrett, R., Berry, M., Chan, T. F., Demmel, J., Donato, J., Dongarra, J., Eijkhout, V., Pozo, R., Romine, C., And Van Der Vorst, H. Templates for the Solution of Linear Systems: Building Blocks for Iterative Methods, 2 ed. SIAM, 1994. 36

[13] Barros, S. R. M. Multigrid methods for two- and three-dimensional Poisson-type equations on the sphere. Journal of Computational Physics 92 (1991), 313-348. 27, 51, 55 
[14] Barros, S. R. M., Dent, D., Isaksen, L., Robinson, G., Mozdzynski, G., And Wollenweber, F. The IFS: a parallel production weather code. Parallel Computing 21 (1995), 1621-1638. 5, 43, 54, 79

[15] Barros, S. R. M., And García, C. I. A global semi-implicit semi-Lagrangian shallow-water model on locally refined grids. Monthly Weather Review 132 (2004), 53-65. 4, 7, 27, 30, 31

[16] Barros, S. R. M., AND García, C. I. A global finite-difference semi-Lagrangian model for the adiabatic primitive equations. Journal of Computational Physics 226 (2007), 1645-1667. 4, 7, 24, 26, 31, 50, 79

[17] Barros, S. R. M., And García, C. I. A global semi-Lagrangian model for the adiabatic primitive equations on locally refined grids. To appear in Computers $\mathscr{E}$ Mathematics with Applications. doi: 10.1016/j.camwa.2015.02.026 (2015). 4

[18] Bates, J. R., Moorthi, S., And Higgins, R. W. A global multilevel atmospheric model using a vector semi-lagrangian finite-difference scheme. Part I: adiabatic formulation. Monthly Weather Review 121 (1993), 244-263. 7

[19] Berger, M., And Colella, P. Local adaptive mesh refinement for shock hydrodynamics. Journal of Computational Physics 82 (1989), 64-84. 4

[20] Berger, M., And Oliger, J. Adaptive mesh refinement for hyperbolic partial differential equations. Journal of Computational Physics 53 (1984), 484-512. 4

[21] BrandT, A. Multi-level adaptive solutions to boundary-value problems. Mathematics of Computation 31, 138 (1977), 333-390. 28

[22] Côté, J. Variable resoution techniques for weather prediction. Meteorology and Atmospheric Physics 63 (1997), 31-38. 2

[23] Côté, J., Roch, M., Staniforth, A., And Fillion, L. A variable-resolution semilagrangian finite-element global model of the shallow-water equations. Monthly Weather Review 121 (1993), 231-243. 3

[24] Côté, J. E. A. The operational CMC-MRB global environmental multiscale (GEM) model. Part I: design and formulation. Monhtly Weather Review 126 (1998), 1373-1395. 3

[25] Davies, T., Cullen, M. J. P., Malcolm, A. J., Mawson, M. H., Staniforth, A., White, A. A., And Wood, N. A new dynamical core for the Met Office's global and regional modelling of the atmosphere. Quarterly Journal of the Royal Meteorological Society 131 (2005), 1759-1782. 3

[26] Ford, R., Glover, M. J., Ham, D. A., Maynard, C. M., Pickles, S. M., Riley, G. D., And Wood, N. Gung Ho: A code design for weather and climate prediction on exascale machines. Advances in Engineering Software (Submitted). 3

[27] Fulton, S., Ciesielski, P. E., And Schubert, W. H. Multigrid methods for elliptic problems: a review. Monthly Weather Review 114 (9986), 943-959. 28

[28] García, C. I. Métodos de refinamento local em modelos globais de previsão numérica do tempo. PhD thesis, Universidade de São Paulo, 2001. 4, 7, 30, 79 
[29] Haltiner, G. J., And Williams, R. T. Numerical Prediction and Dynamic Meteorology. Wiley, 1980. 7

[30] Holton, J. R. An introduction to dynamical meteorology. Elsevier, 2004. 7

[31] Jablonowski, C. Adaptive grids in weather and climate modeling. PhD thesis, University of Michigan, 2004. 4

[32] Jablonowski, C., And Williamson, D. A baroclinic instability test case for atmospheric model dynamical cores. Quarterly Journal of the Royal Meteorological Society 132 (2006), 2943-2975. 56, 57

[33] Jablonowski, C., and Williamson, D. A baroclinic wave test case for dynamical cores of general circulation models: Model intercomparisons. Tech. rep., NCAR Technical Note, 2006. 5, 56, 57, 59, 61

[34] Majewski, D., Liermann, D., Prohl, P., Ritter, B., Buchhold, M., Hanisch, T., Paul, G., Wergen, W., And Baumgardner, J. The operational global icosahedralhexagonal gridpoint model gme: Description and high-resolution tests. Monthly Weather Review 130 (2002), 319-338. 2

[35] Met Office. ENDGame: A new dynamical core for seamless atmospheric prediction, 2014. Disponível em

http://www.metoffice.gov.uk/media/pdf/s/h/ENDGameGOVSci_v2.0.pdf. 3

[36] Michalakes, J. A runtime system library for parallel finite difference models with nesting. Tech. rep., Mathematics and Computer Science Division, Argonne National Laboratory, Argonne, IL, 1995. 40

[37] Moorthi, S., Higgins, R. W., And Bates, J. R. A global multilevel atmospheric model using a vector semi-lagrangian finite-difference scheme. Part II: version with physics. Monthly Weather Review 123 (1995), 1523-1541. 7

[38] National Center for Environmental Prediction (NCEP). Weather Research E Forecasting - NMM Version 3 Modeling System User's Guide, 2014. 2

[39] Polvani, L. M., Scott, R. K., and Thomas, S. J. Numerically converged solutions of the global primitive equations for testing the dynamical core of atmospheric CGMs. Monthly Weather Review 132 (2004), 2539-2552. 56

[40] Ritchie, H. Semi-Lagrangian advection on a Gaussian grid. Monthly Weather Review 115 (1987), 608-619. 13, 14, 28

[41] Ritchie, H. Application of the semi-Langrangian method to a spectral model of the shallow water equations. Monthly Weather Review 116 (1988), 1587-1598. 17, 28

[42] Ritchie, H. Application of the semi-Lagrangian method to a multilevel spectral primitive-equations model. Q. J. R. Met. Soc. 117 (1991), 91-106. 13, 16

[43] Ritchie, H., And Tanguay, M. A comparison of spatially averaged Eulerian and semi-Lagrangian treatments of mountains. Monthly Weather Review 124 (1996), 167181. 10 
[44] Ritchie, H., Temperton, C., Simmons, A., Hortal, M., Davies, T., Dent, D., AND HAmrud, M. Implementation of the Semi-Lagrangian method in a high-resolution version of the ECMWF forecast model. Monthly Weather Review 123 (1995), 489-514. 13

[45] Skamarock, W., AND Klemp, J. Adaptive grid refinement for two-dimensional and three-dimensional nonhydrostatic atmospheric flow. Monthly Weather Review 121 (1993), 788-804. 4

[46] Skamarock, W., Oliger, J., And Street, R. Adaptive grid refinement for numerical weather prediction. Journal of Computational Physics 80 (1989), 27-60. 4

[47] Staniforth, A., And Côté, J. Semi-Lagrangian integration schemes for atmospheric models - a review. Monthly Weather Review 119 (1992), 2206-2223. 12

[48] Staniforth, A., White, A., Wood, N., Thuburn, J., Zerroukat, M., Cordero, E., Davies, T., And Diamantakis, M. Joy of U.M. 6.3 - Model formulation. Met Office, 2006. 3

[49] Staniforth, A., And Wood, N. Aspects of the dynamical core of a nonhydrostatic, deep-atmosphere, unified weather and climate-prediction model. Journal of Computational Physics 227 (2008), 3445-3464. 3

[50] StÜben, K., And Trottenberg, U. Multigrid methods: Fundamental algorithms, model problem analysis and applications. In Lecture Notes in Mathematics (1982), W. Hackbusch and U. Trottenberg, Eds., vol. 960, Springer-Verlag, Berlin. 28, 50

[51] Toviessi, J., Patoine, A., And Lemay, G. Parallel computing at Canadian meteorological centre. In Developments in Teracomputing: Proceedings of the 9th ECMWF Workshop on the use of High Performance Computing in Meteorology (2001), W. Zwieflhofer and N. Kreitz, Eds., World Scientific Publishing. 3

[52] Trottenberg, U., Oosterlee, C., And Schüller, A. Multigrid. Elsevier. Academic Press, 2001. 28, 52

[53] Wilkinson, B., And Allen, M. Parallel programming. Techniques and applications using networked workstations and parallel computers. Prentice Hall, 1999. 71

[54] Williams, P. A proposed modification to the Robert-Asselin time filter. Monthly Weather Review 137 (2009), 2538-2546. 57

[55] Williams, P. The RAW filter: an improvement to the Robert-Asselin filter in semiimplicit integrations. Monthly Weather Review 139 (2011), 1996-2007. 57

[56] YessAD, K. Basic about ARPEGE/IFS, ALADIN and AROME in the cycle 41 of ARPEGE/IFS. Meteo-France, 2014. 3

[57] Zhu, X., And Oliger, J. Data structures and implementation of composite adaptive grid methods. Tech. rep., Research Institute for Advanced Computer Science, Standford University, 1995. 36 